\title{
On a singular point of Briot-Bouquet type of a system of two ordinary nonlinear differential equations.
}

\author{
By \\ Masahiro IWANO
}

\section{Statement of the problem.}

The singular point of Briot-Bouquet type of an ordinary nonlinear differential equation

$$
x^{\sigma+1} \frac{d y}{d x}=f(x, y), \quad f(0,0)=0, \quad \sigma \geqq 0,
$$

where $f(x, y)$ is a holomorphic function of $(x, y)$ in a neighborhood of $(0,0)$, has been studied by diverse authors since Ch. Briot and J.-C. Bouquet, and the most complete results were obtained by M. Hukuhara [1], [2], [3], [4], [5] and [6]. One of the most remarkable results occurs in the study of the case when $\sigma=0$ and $\lambda=f_{y}^{\prime}(0,0)$ vanishes, and this played an important role in the theory of T. Kimura [1], which comes in close contact with work of J. Malmquist [1].

The singular points $x=0$ of a system of ordinary nonlinear differential equations of the form

$$
x \frac{d y_{j}}{d x}=f_{j}\left(x, y_{1}, \cdots, y_{n}\right) \quad(j=1,2, \cdots, n),
$$

(where $f_{j}(x, y)$ are holomorphic functions of $(x, y)$ in a neighborhood of $(0,0)$ and vanish there), are usually said to be of BriotBouquet type. Recently, a classical result concerning the expan-

Received April 23, 1966.

Communicated by M. Hukuhara.

Sponsored in part by the Mathematics Research Center, United States Army, Madison, Wisconsin, under Contract No. : DA-11-022-ORD-2059, and in part by the Japan Gakujutsu Shinkokai Foundation. 
sions of particular solutions in uniformly convergent power series of certain functions has been extended by the author (M. Iwano [3]). However, it has not yet been studied when the eigenvalues of the matrix $F$ formed by $\left\{\partial f_{j} / \partial y_{k}(0,0)\right\}$ are all zero. In this paper, we shall study the case that $F$ is the zero matrix for a system of two differential equations.

Let there be given a system of two ordinary nonlinear differential equations of the form

$$
x \frac{d y}{d x}=f(x, y, z), \quad x \frac{d z}{d x}=g(x, y, z),
$$

where we suppose that

1) both $f(x, y, z)$ and $g(x, y, z)$ are functions holomorphic and bounded in $(x, y, z)$ in

$$
|x|<a, \quad|y|<b, \quad|z|<b
$$

and vanishing at $x=y=z=0$;

2) the functions $f(0, y, z)$ and $g(0, y, z)$ have uniformly convergent expansions of the form

$$
\left\{\begin{array}{l}
f(0, y, z)=y^{m+1} z^{n}\left(\alpha+\Sigma^{(1)} \alpha_{k l} y^{k} z^{l}\right) \\
g(0, y, z)=y^{m} z^{n+1}\left(\beta+\sum^{(1)} \beta_{k l} y^{k} z^{l}\right),
\end{array}\right.
$$

and $m$ and $n$ are nonnegative integers not simultaneously zero; 3) $\alpha$ and $\beta$ are nonzero complex constants such that

$$
\operatorname{Re} \frac{\alpha}{m \alpha+n \beta}>0, \quad \operatorname{Re} \frac{\beta}{m \alpha+n \beta}>0 .
$$

What we intend here is the construction of a solution depending on two arbitrary constants.

In the case when $f(x, y, z)$ and $g(x, y, z)$ contain respectively the terms $y^{m+1} z^{n}$ and $y^{m} z^{n+1}$ as a factor, M. Hukuhara [7] constructed a formal solution of (1.1) depending on two arbitrary parameters without the condition 3 ), but its analytical meaning

1) $\Sigma^{(N)} d_{k_{1}} \cdots k_{s} y_{1} k_{1} \cdots y_{s} k_{s}$ denotes the summation for all the arrangements $\left(k_{1}, \cdots, k_{s}\right)$ of $s$ nonnegative integers $k_{1}, \ldots, k_{s}$ such that $k_{1}+\cdots+k_{s} \geqq N$. 
was not given there.

The author wishes to express his cordial thanks to Professors Wolfgang Wasow and Tosiya Saito for their kind guidance and valuable advice.

\section{Part I. Preliminaries.}

Chapter I. Formal transformation.

\section{Result concerning a formal reduction.}

Since the second members of the differential equations (1.1) are holomorphic functions of $(x, y, z)$ in the domain (1.2), they can be expanded in powers of $x, y, z$ as follows:

$$
f=\sum x^{j} y^{k} z^{l} f_{j k l}, \quad g=\sum x^{j} y^{k} z^{l} g_{j k l}
$$

with

$$
\begin{array}{ll}
f_{0, k+n n+1, l+n}=\alpha_{k l}, & g_{0, k+n n, l+n+1}=\beta_{k l}, \\
f_{0, m+1, n}=\alpha, & g_{0, m, n+1}=\beta .
\end{array}
$$

Moreover, we can suppose without loss of generality that

$$
f_{100}=0, \quad g_{100}=0 .
$$

Indeed, if it is not true, it is sufficient to make a linear transformation of the form

$$
y=Y+x f_{100}, \quad z=Z+x g_{100} .
$$

According to our usual method, we try to transform the second members of (1.1) into as simple a form as possible.

The aim of this chapter is to establish the following:

Theorem 1. CASE A $(\alpha: \beta \neq a$ rational number $)$. There exists a formal transformation

$$
\begin{cases}y \sim u+\sum^{(2)} P_{j_{k l}} x^{j} u^{k} v^{l}, & P_{00 z}=0, \\ z \sim v+\sum^{(2)} Q_{j \kappa l} x^{j} u^{k} v^{l}, & Q_{0 \kappa 0}=0,\end{cases}
$$

which transforms formally the system (1.1) into a system

$$
\left\{\begin{array}{l}
x u^{\prime}=u^{m+1} v^{n}\left(\alpha+\alpha^{\prime} u^{m} v^{n}\right),{ }^{2)} \\
x v^{\prime}=u^{m} v^{n+1}\left(\beta+\beta^{\prime} u^{m} v^{n}\right) .
\end{array}\right.
$$

2) The symbol' denotes the derivative with the obvious exception $\alpha^{\prime}, \beta^{\prime}$. 
CASE B ( $\alpha: \beta=a$ positive rational number). In this case, there exist positive integers $\mu, \nu$ and a complex constant $\gamma$ such that

$$
\frac{\alpha}{\mu}=\frac{\beta}{\nu}=\gamma \quad(\mu \text { and } \nu \text { are mutually prime }) .
$$

Then there exists a formal transformation of the type (2.2) which reduces formally (1.1) to

$$
\left\{\begin{array}{l}
x u^{\prime}=\mu u^{m+1} v^{n}\left(\gamma+u^{m} v^{n} \sum_{h}\left(u^{\nu} v^{-\mu}\right)^{h} \gamma_{h}\right), \\
x v^{\prime}=\nu u^{m} v^{n+1}\left(\gamma+u^{m} v^{n} \sum_{h}\left(u^{\nu} v^{-\mu}\right)^{n} \gamma_{h}\right),
\end{array}\right.
$$

where the summation is to be taken over all integers $h$ such that $-m / \nu \leqq h \leqq n / \mu$ and $m+\nu h$ and $n-\mu h$ are nonnegative integers not simultaneously zero.

Remark. In the case when $\alpha: \beta$ is negative rational, the inequalities (1.4) are not satisfied. Therefore we omit this case.

\section{Formal transformation of the first kind.}

To prove Theorem 1, we consider a formal transformation of the form

$$
\left\{\begin{array}{l}
y=y_{N}+\sum_{(N)} p_{j k l}^{(N)} x^{j} y_{N}^{k} z_{N}^{l},{ }^{3)} \\
z=z_{N}+\sum_{(N)} q_{j k l}^{(N)} x^{j} y_{N}^{k} z_{N}^{l}
\end{array}\right.
$$

Let

$$
\left\{\begin{array}{l}
x y_{N}^{\prime}=\sum f_{j k l}^{(N)} x^{j} y_{N}^{k} z_{N}^{l} \\
x z_{N}^{\prime}=\sum g_{j k l}^{(N)} x^{j} y_{N}^{k} z_{N}^{l}
\end{array}\right.
$$

be the system derived from (1.1) by the transformation (3.1).

The inverse transformation of (3.1) can be written as

$$
\left\{\begin{array}{l}
y_{N}=y-\sum_{(N)} p_{j k l}^{(N)} x^{j} y^{k} z^{l}+[x, y, z]_{2 N-1},{ }^{4)} \\
z_{N}=z-\sum_{(N)} q_{j k l}^{(N)} x^{j} y^{k} z^{l}+[x, y, z]_{2 N-1}
\end{array}\right.
$$

3) $\Sigma_{(N)}$ denotes the summation to be taken over all the arrangements $(j, k, l)$ of nonnegative integers $j, k, l$ such that $j+k+l=N$.

4) The symbol $[x, y, z]_{M}$ represents the formal power series of $x, y, z$ lacking the terms of degree less than $M$. 
Differentiating (3.3) term by term, we have the following relations

$$
\left\{\begin{array}{r}
x y_{N}^{\prime}=x y^{\prime}-\left[\sum_{(N)} p_{j k l}^{(N)}\left(j+k \frac{x y^{\prime}}{y}+l \frac{x z^{\prime}}{z}\right) x^{j} y^{k} z^{l}\right. \\
\left.+x \frac{d}{d x}[x, y, z]_{2 N-1}\right] \\
x z_{N}^{\prime}=x z^{\prime}-\left[\sum_{(N)} q_{j k l}^{(N)}\left(j+k \frac{x y^{\prime}}{y}+l \frac{x z^{\prime}}{z}\right) x^{j} y^{k} z^{l}\right. \\
\left.+x \frac{d}{d x}[x, y, z]_{2 N-1}\right]
\end{array}\right.
$$

where the terms $x y^{\prime}$ and $x z^{\prime}$ must be replaced by the first and the second expressions of (2.1) respectively.

Observing that we have

$$
f_{j k l}=0, \quad g_{j k l}=0 \quad(j+k+l \leqq 1),
$$

we can derive easily the following relations

$$
f_{j k l}^{(N)}=f_{j k l}, \quad g_{j k l}^{(N)}=g_{j k l} \quad(j+k+l<N)
$$

and

$$
f_{j k l}^{(N)}=f_{j k l}-j p_{j k l}^{(N)}, \quad g_{j k l}^{(N)}=g_{j k l}-j q_{j k l}^{(N)} \quad(j+k+l=N) .
$$

Hence we can determine the values of the coefficients $p_{j k l}^{(N)}$ and $q_{j k l}^{(N)}$ so that $f_{j k l}^{(N)}$ and $g_{j k l}^{(N)}$ are reduced to 0 for $j+k+l=N$ except for $j=0$. For $j=0$, we put

$$
p_{j k l}^{(N)}=0, \quad q_{j k l}^{(N)}=0 \quad(j=0) .
$$

Then it is easy to see that the expressions appearing in the brackets denoted by [] in the right-hand members of (3.4) contain $x$ as a factor. From this it immediately follows that

$$
f_{0 k l}^{(N)}=f_{0 k l}, \quad g_{0 k l}^{(N)}=g_{0 k l} .
$$

Thus, applying successively transformations of the form

$$
\left\{\begin{array}{lc}
y=y_{1}, \quad z=z_{1} ; & \\
y_{1}=y_{2}+\sum_{(2)} p_{j k L}^{(2)} x^{j} y_{2}^{k} z_{2}^{l}, & z_{1}=z_{2}+\sum_{(2)} q_{j k l}^{(2)} x^{j} y_{2}^{k} z_{2}^{l} ; \\
\ldots \ldots \ldots \ldots \ldots \ldots \ldots & \ldots \ldots \ldots \ldots \ldots \ldots \ldots
\end{array}\right.
$$




$$
\begin{array}{cc}
y_{N-1}=y_{N}+\sum_{(N)} p_{j k l}^{(N)} x^{j} y_{N}^{k} z_{N}^{l}, & z_{N-1}=z_{N}+\sum_{(N)} q_{j k l}^{(N)} x^{j} y_{N}^{k} z_{N}^{l} \\
\ldots \ldots \ldots \ldots \ldots \ldots . & \ldots \ldots \ldots \ldots \ldots \ldots .
\end{array} ;
$$

we obtain the following:

Lemma 1. 1. Let there be given the following system

$$
x y^{\prime}=\sum f_{j k l} x^{j} y^{k} z^{l}, \quad x z^{\prime}=\sum g_{j k l} x^{j} y^{k} z^{l}
$$

with

$$
f_{j k l}=g_{j k l}=0 \quad \text { for } j+k+l \leqq 1 .
$$

Then there exists a formal transformation of the form

$$
\begin{gathered}
\left\{\begin{array}{c}
y \sim \eta+\sum^{(2)} p_{j k l} x^{j} \eta^{k} \zeta^{l}, \\
z \sim \zeta+\Sigma^{(2)} q_{j k l} x^{j} \eta^{k \zeta} \zeta^{2}
\end{array}\right. \\
p_{0 k l}=q_{0 k l}=0
\end{gathered}
$$

such that the system (1.1) is formally reduced to

$$
x \eta^{\prime}=\sum f_{k l} \eta^{k \zeta} \zeta^{l}, \quad x \zeta^{\prime}=\sum g_{k l} \eta^{k} \zeta^{l}
$$

with

$$
f_{k l}=f_{0 k l}, \quad g_{k l}=g_{0 k l} .
$$

4. Formal transformation of the second kind.

We see by Lemma 1.1 that the power series appearing in the right-hand members of (3.5) coincide with the expressions (1.3). Hence, the system (3.6) is written as

$$
\left\{\begin{array}{l}
x \eta^{\prime}=\eta^{m+1} \zeta^{n}\left(\alpha+\Sigma^{(1)} \alpha_{k l} \eta^{k} \zeta^{l}\right), \\
x \zeta^{\prime}-\eta^{m \zeta^{n+1}}\left(\beta+\Sigma^{(1)} \beta_{k l} \eta^{k \zeta} \zeta^{l}\right),
\end{array}\right.
$$

where the power series appearing in the right-hand members are clearly uniformly convergent in a neighborhood of $(0,0)$.

Now we consider a further transformation of the form

$$
\left\{\begin{array}{l}
\eta=u_{N}\left(1+\sum_{(N)} p_{k l}^{(N)} u_{N}^{k} v_{N}^{l}\right), \\
\zeta=v_{N}\left(1+\sum_{(N)} q_{k l}^{(N)} u_{N}^{k} v_{N}^{l}\right) .
\end{array}\right.
$$

Then it is easily found that the transformed equations have also the same form as (4.1) as follows: 


$$
\left\{\begin{array}{l}
x u_{N}^{\prime}=u_{N}^{m+1} v_{N}^{n}\left(\alpha+\sum^{(1)} \alpha_{k l}^{(N)} u_{N}^{k} v_{N}^{l}\right), \\
x v_{N}^{\prime \prime}=u_{N}^{m} v_{N}^{n+1}\left(\beta+\Sigma^{(1)} \beta_{k l}^{(N)} u_{N}^{k} v_{N}^{l}\right)
\end{array}\right.
$$

The inverse transformation of (4.2) can be written as

$$
\left\{\begin{array}{l}
u_{N}=\eta\left(1-\sum_{(N)} p_{k l}^{(N)} \eta^{k} \zeta^{2}+[\eta, \zeta]_{2 N}\right) \\
v_{N}=\zeta\left(1-\sum_{(N)} q_{k l}^{(N)} \eta^{k} \zeta^{2}+[\eta, \zeta]_{2 N}\right)
\end{array}\right.
$$

Differentiation yields equalities of the form

$$
\begin{aligned}
x u_{N}^{\prime}=x \eta^{\prime}-\eta \Sigma_{(N)} p_{k l}^{(N)}[ & \left.(k+1) \frac{x \eta^{\prime}}{\eta}+l \frac{x \zeta^{\prime}}{\zeta}\right] \eta^{k \zeta \zeta^{l}} \\
& +x \frac{d}{d x}\left\{\eta \cdot[\eta, \zeta]_{2 N}\right\} \\
x v_{N}^{\prime}=x \zeta^{\prime}-\zeta \sum_{(N)} q_{k l}^{(N)}[ & {\left[k \frac{x \eta^{\prime}}{\eta}+(l+1) \frac{x \zeta^{\prime}}{\zeta}\right] \eta^{k \zeta} \zeta^{l} } \\
& +x \frac{d}{d x}\left\{\zeta \cdot[\eta, \zeta]_{2 N}\right\}
\end{aligned}
$$

and we have, by (4.1),

$$
\begin{aligned}
x u_{N}^{\prime}=\eta^{m+1} \zeta^{n}(\alpha & \left.+\sum^{(1)} \alpha_{k l} \eta^{k \zeta} \zeta^{l}\right)-\eta^{m+1} \zeta^{n} \sum_{(N)} p_{k l}^{(N)}\{(k+1) \alpha \\
& \left.+l \beta+[\eta, \zeta]_{1}\right\} \eta^{k} \zeta^{l}+\eta^{m+1} \zeta{ }^{n}[\eta, \zeta]_{2 N}, \\
x v_{N}^{\prime}=\eta^{m} \zeta^{n+1}(\beta & \left.+\sum^{(1)} \beta_{k l} \eta^{k \zeta l} \zeta^{l}\right)-\eta^{m} \zeta^{n+1} \sum_{(N)} q_{k l}^{(N)}\{k \alpha+(l+1) \beta \\
& \left.+[\eta, \zeta]_{1}\right\} \eta^{k} \zeta^{l}+\eta^{m} \zeta^{n+1}[\eta, \zeta]_{2 N} .
\end{aligned}
$$

Substituting the expressions (4.2) for $\eta$ and $\zeta$ into the equations above, we can easily derive the following relations

$$
\alpha_{k l}^{(N)}=\alpha_{k l}, \quad \beta_{k l}^{(N)}=\beta_{k l} \quad(k+l<N)
$$

and

(4. 5) $\left\{\begin{array}{l}\alpha_{k l}^{(N)}=\alpha_{k l}+\{(m-k) \alpha-l \beta\} p_{k l}^{(N)}+n \alpha q_{k l}^{(N)}, \\ \beta_{k l}^{(N)}=\beta_{k l}+m \alpha p_{k l}^{(N)}+\{-k \alpha+(n-l) \beta\} q_{k l}^{(N)},\end{array} \quad(k+l=N)\right.$.

A simple calculation shows us that the determinant of the matrix formed by the coefficients of $p_{k l}^{(N)}$ and $q_{k l}^{(N)}$ in (4.5) is given by

$$
\begin{aligned}
\Delta(k, l) & =\left|\begin{array}{cc}
(m-k) \alpha-l \beta & n \alpha \\
m \alpha & -k \alpha+(n-l) \beta
\end{array}\right| \\
& =(k \alpha+l \beta)[(k-m) \alpha+(l-n) \beta] .
\end{aligned}
$$


Hence we can determine the values of the coefficients $p_{k l}^{(N)}$ and $q_{k l}^{(N)}$ so that $\alpha_{k l}^{(N)}$ and $\beta_{k l}^{(N)}$ become zero for $k+l=N$ if $\Delta(k, l) \neq 0$.

To discuss the case when $\Delta(k, l)=0$, we distinguish the following two cases :

Case A. $\alpha: \beta \neq$ a rational number,

Case B. $\alpha: \beta=$ a positive rational number.

I. Case A. Since $k+l \geqq 1$, we see that the equation $\Delta(k, l)=0$ holds if and only if $k-m=0$ and $l-n=0$. This means that we have $\Delta(k, l)=0$ for $(k, l)=(m, n)$ only.

II. Case B. In this case, there exist positive integers $\mu, \nu$ and a complex constant $\gamma$ such that

$$
\frac{\alpha}{\mu}=\frac{\beta}{\nu}=\gamma \quad(\mu \text { and } \nu \text { are mutually prime }) .
$$

Hence, the equation $\Delta(k, l)=0$ will be reduced to

$$
\mu(k-m)+\nu(l-n)=0,
$$

i. e.

$$
\mu(k-m)=\nu(n-l) .
$$

Since $\mu$ and $\nu$ are supposed to be mutually prime, there exists an integer $h$ such that we have

$$
k-m=\nu h, \quad n-l=\mu h
$$

for every $(k, l)$. Noticing that $k$ and $l$ are nonnegative integers not simultaneously zero and $\mu, \nu$ are positive integers, we see that $h$ must satisfy the inequalities

$$
-\frac{m}{\nu} \leqq h \leqq \frac{n}{\mu} \quad \text { or } \quad-\frac{n}{\mu} \leqq h \leqq \frac{m}{\nu} .
$$

From this it follows at once that the arrangements $(k, l)$ for which $\Delta(k, l)=0$ are finite in number, and for such arrangements $(k, l)$ we can determine the values of the coefficients $p_{k l}^{(N)}$ and $q_{k l}^{(N)}$ so that the values of $\alpha_{k l}^{(N)}$ and $\beta_{k l}^{(N)}$ (given by (4.5)) satisfy the relations 


$$
\frac{\alpha_{k l}^{(N)}}{\mu}=\frac{\beta_{k l}^{(N)}}{\nu}\left(=\gamma_{h}\right)
$$

Thus, applying successively transformations of the form

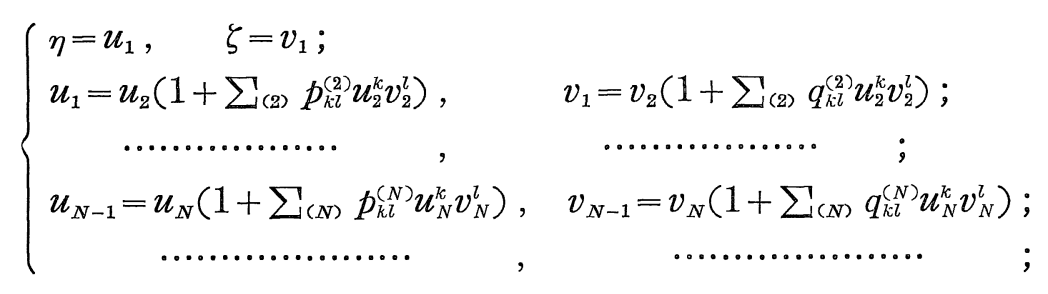

we can obtain the following:

Lemma 1.2. We can determine the values of coefficients $p_{k l}$ and $q_{k l}$ of a formal transformation

$$
\left\{\begin{array}{l}
\eta \sim u\left(1+\sum^{(1)} p_{k l} u^{k} v^{l}\right) \\
\zeta \sim v\left(1+\Sigma^{(1)} q_{k l} u^{k} v^{l}\right)
\end{array}\right.
$$

so that the system (4.1) is formally reduced to a system of the form (2.3):

$$
\left\{\begin{array}{l}
x u^{\prime}=u^{m+1} v^{n}\left(\alpha+\beta^{\prime} u^{m} v^{n}\right), \\
x v^{\prime}=u u^{m} v^{n+1}\left(\beta+\beta^{\prime} u^{m} v^{n}\right),
\end{array}\right.
$$

or to a system of the form $\left(2.3^{\prime}\right)$ :

$$
\left\{\begin{array}{l}
x u^{\prime}=\mu u^{m+1} v^{n}\left(\gamma+u^{m} v^{n} \sum\left(u^{\nu} v^{-\mu}\right)^{h} \gamma_{h}\right), \\
x v^{\prime}=\nu u^{m} v^{n+1}\left(\gamma+u^{m} v^{n} \sum\left(u^{\nu} v^{-\mu}\right)^{h} \gamma_{h}\right),
\end{array}\right.
$$

according as we have Case A or Case B.

In the latter case, the index $h$ runs over all the integers satisfying the inequalities

$$
-m / \nu \leqq h \leqq n / \mu
$$

such that $m+\nu h$ and $n-\mu h$ are nonnegative integers not simultaneously zero.

By Lemma 1.1 and Lemma 1.2 we see that, applying successively two transformations of the forms (3.5) and (4.7), the system (1.1) is formally transformed into a system of the form (2.3) or (2. $\left.3^{\prime}\right)$ according as we have Case A or Case B. 
Thus Theorem 1 has been proved.

Chapter II. General solution of the reduced system.

5. Results concerning integration of the reduced system.

The quantities $\alpha, \beta$ and $m \alpha+n \beta$ are supposed to be all nonzero. To simplify the description, we shall assume that $\alpha^{\prime}, \beta^{\prime}$ and $m \alpha^{\prime}+m \beta^{\prime}$ are also different from zero. We have already proved that the given system (1.1) can be formally reduced to one of the following forms:

$$
\begin{gathered}
\left\{\begin{array}{l}
x u^{\prime}=u^{m+1} v^{n}\left(\alpha+\alpha^{\prime} u^{m} v^{n}\right), \\
x v^{\prime}=u^{m} v^{n+1}\left(\beta+\beta^{\prime} u^{m} v^{n}\right),
\end{array}\right. \\
\left\{\begin{array}{l}
x u^{\prime}=\mu u^{m+1} v^{n}\left(\gamma+u^{m} v^{n} \sum \gamma_{h}\left(u^{\nu} v^{-\mu}\right)^{n}\right), \\
x v^{\prime}=\nu u^{m} v^{n+1}\left(\gamma+u^{m} v^{n} \sum \gamma_{h}\left(u^{\nu} v^{-\mu}\right)^{n}\right) .
\end{array}\right.
\end{gathered}
$$

As can be easily verified, direct solution of these reduced systems by quadratures is generally impossible. However, if we introduce an auxiliary independent variable

$$
w=u^{m} v^{n},
$$

we can obtain a parametric representation of the general solution of the reduced system.

The aim of this chapter is to prove the following three theorems :

Theorem 2.1. If we introduce an auxiliary independent variable $w$ by the relation (5.1), the reduced system will be reduced to a system of the form

$$
\left\{\begin{array}{l}
w^{2} \frac{d x}{d w}=x\left[\frac{1}{m \alpha+n \beta+\left(m \alpha^{\prime}+n \beta^{\prime}\right) w}\right], \\
w \frac{d u}{d w}=u\left[\frac{\alpha+\alpha^{\prime} w}{m \alpha+n \beta+\left(m \alpha^{\prime}+n \beta^{\prime}\right) w}\right], \quad\left(w=u^{m} v^{n}\right) \\
w \frac{d v}{d w}=v\left[\frac{\beta+\beta^{\prime} w}{m \alpha+n \beta+\left(m \alpha^{\prime}+n \beta^{\prime}\right) w}\right],
\end{array}\right.
$$

or 


$$
\left\{\begin{aligned}
w^{2} \frac{d x}{d w} & =x\left[\frac{1}{(m \mu+n \nu)\left(\gamma+\gamma^{\prime} w\right)}\right], \\
w \frac{d u}{d w} & =u\left[\frac{\mu}{m \mu+n \nu}\right], \quad\left(w=u^{m} v^{n}\right) \\
w \frac{d v}{d w} & =v\left[\frac{\nu}{m \mu+n \nu}\right], \\
\gamma^{\prime} & =\sum \gamma_{h} \cdot\left(u^{\nu} v^{-\mu}\right)^{h},
\end{aligned}\right.
$$

according as we have Case A or Case $\mathrm{B}$.

The general solution of the reduced system (2.3) or (2. $\left.3^{\prime}\right)$ can be expressed as

$$
\left\{\begin{array}{l}
x=C_{1}\left(1+\frac{m \alpha+n \beta}{\left(m \alpha^{\prime}+n \beta^{\prime}\right) w}\right)^{\frac{m \alpha^{\prime}+n \beta^{\prime}}{(m \alpha+n \beta)^{2}}} \exp \left(-\frac{1}{(m \alpha+n \beta) w}\right) \\
u=C_{2}^{n} w^{\mathfrak{A}}\left((m \alpha+n \beta)+\left(m \alpha^{\prime}+n \beta^{\prime}\right) w\right)-n\left(\mathfrak{H} \mathfrak{B}^{\prime}-\mathfrak{H}^{\prime} \mathfrak{B}\right) \\
v=C_{2}^{-m} w^{\mathfrak{P}}\left((m \alpha+n \beta)+\left(m \alpha^{\prime}+n \beta^{\prime}\right) w\right) m\left(\mathfrak{A} \mathfrak{B}^{\prime}-\mathfrak{\mathfrak { U } ^ { \prime } \mathfrak { B } )},\right.
\end{array}\right.
$$

or

$$
\left\{\begin{array}{l}
x=C_{1}\left(1+\frac{\gamma}{\gamma^{\prime} w}\right)^{\frac{\gamma^{\prime}}{\gamma^{2((m+n \nu)}}} \exp \left(-\frac{1}{\gamma(m \mu+n \nu) w}\right) \\
u=C_{2}^{n} w^{\mathfrak{A}} \\
v=C_{2}^{-m} w^{\mathfrak{B}}
\end{array}\right.
$$

according as we have Case A or Case B. Here we adopted the following notations for simplicity

$$
\begin{cases}\mathfrak{U}=\frac{\alpha}{m \alpha+n \beta}, & \mathfrak{U}^{\prime}=\frac{\alpha^{\prime}}{m \alpha^{\prime}+n \beta^{\prime}} \\ \mathfrak{B}=\frac{\beta}{m \alpha+n \beta}, & \mathfrak{B}^{\prime}=\frac{\beta^{\prime}}{m \alpha^{\prime}+n \beta^{\prime}}\end{cases}
$$

or

$$
\mathfrak{U}=\mathfrak{U}^{\prime}=\frac{\mu}{m \mu+n \nu}, \quad \mathfrak{B}=\mathfrak{B}^{\prime}=\frac{\nu}{m \mu+n \nu},
$$

according as we have Case A or Case $\mathrm{B}$.

Remark. Let $x=X^{*}(w), u=U^{*}(w), v=V^{*}(w)$ be the holomorphic solution of the system (5.2) or $\left(5.2^{\prime}\right)$ (where the expression $\gamma^{\prime} w$ should be replaced by $\left.\sum_{h \neq 0} \gamma_{h} u^{\nu h+m} v^{-\mu h+n}+\gamma_{0} w\right)$ satis- 
fying the initial condition: $x=x_{0}, u=u^{0}, v=v^{0}$ at $w=w^{0}$. Then, as can be easily verified from a direct calculation, these functions can be a solution of the reduced system (2.3) or (2.3') if and only if the relation $U^{*}(w)^{m} V^{*}(w)^{n}=w$ holds. However, this identity is equivalent to $\left(u^{0}\right)^{m}\left(v^{0}\right)^{n}=w^{0}$. Indeed, a simple calculation shows us that $U^{*}(w)^{m} V^{*}(w)^{n}-w$ is the solution of a linear differential equation of the form $w \frac{d y}{d w}=y$.

If we want to express $u$ and $v$ as functions of $x$, we must eliminate $w$ from the relations (5.3) or $\left(5.3^{\prime}\right)$. To do this, it is convenient to use a certain transcendental function introduced by M. Hukuhara [1], and the result can be stated as follows:

Theorem 2. 2. Let $Y=S_{\mathcal{C}}(X)$ be defined implicitly by

$$
X=Y-\log (Y+1)
$$

and consider the branch of $\mathfrak{W}(X)$ such that $\mathfrak{S}(X)-X-\log X$ vanishes at $X=\infty$. Then the general solution of the reduced system (2.3) or ( $\left.2.3^{\prime}\right)$ can be written as

$$
\left\{\begin{array}{c}
u=C_{2}^{n}\left(\frac{m \alpha^{\prime}+n \beta^{\prime}}{m \alpha+n \beta}\right)^{-\mathfrak{A}} \mathfrak{S}\left(X+C_{1}^{0}\right)-\mathfrak{A} \\
\times\left[1+\mathfrak{S}\left(X+C_{1}^{0}\right)^{-1}\right]-n\left(\mathfrak{H} \mathfrak{B}^{\prime}-\mathfrak{A} \mathfrak{H}^{\prime}\right), \\
v=C_{2}^{-m}\left(\frac{m \alpha^{\prime}+n \beta^{\prime}}{m \alpha+n \beta}\right)^{-\mathfrak{B}} \mathfrak{S}\left(X+C_{1}^{0}\right)-\mathfrak{B} \\
\times\left[1+\mathfrak{S}\left(X+C_{1}^{0}\right)^{-1}\right] m\left(\mathfrak{H} \mathfrak{B}^{\prime}-\mathfrak{H}{ }^{\prime} \mathfrak{B}\right), \\
X=-\frac{(m \alpha+n \beta)^{2}}{m \alpha^{\prime}+n \beta^{\prime}} \log x,
\end{array}\right.
$$

or

$$
\left\{\begin{array}{c}
u=\left(\frac{\gamma^{\prime}}{\gamma} C_{2}^{\frac{n}{\mu}}\right)^{-\mathfrak{A}} \mathfrak{S}\left(X+C_{1}^{0}\right)-\mathfrak{H}, \\
v=\left(\frac{\gamma^{\prime}}{\gamma} C_{2}^{-\frac{m}{\nu}}\right)^{-\mathfrak{B}} \mathfrak{S}\left(X+C_{1}^{0}\right)-\mathfrak{B}, \\
X=-\frac{\gamma^{2}\left(m_{\mu}+n \nu\right)}{\gamma^{\prime}} \log x,
\end{array}\right.
$$

according as we have Case A or Case $\mathrm{B}$. 
The nature of the function $x=\tilde{x}(w)$ defined by the first relation of (5.3) or (5.3') and of its inverse function is clarified by the Theorem below:

Theorem 2.3. We denote by $D$ and $D^{*}$ the following angular regions in the complex $w$-plane

$$
\begin{aligned}
D & =\left\{w: \theta_{-}+2 \delta_{0} \leqq \arg w \leqq \theta_{+}-2 \delta_{0}, 0<|w|<c\right\}, \\
D^{*} & =\left\{w: \theta_{-}-\pi+\delta_{0} \leqq \arg w \leqq \theta_{+}+\pi-\delta_{0}, 0<|w|<c\right\},
\end{aligned}
$$

where

$$
\theta_{ \pm}=-\arg (m \alpha+n \beta) \pm \frac{\pi}{2}
$$

or

$$
\theta_{ \pm}=-\arg \gamma \pm \frac{\pi}{2}
$$

according as we have Case A or Case $\mathrm{B}, \delta_{0}$ being an arbitrarily fixed sufficiently small positive constant.

Let $x_{0}$ and $w^{0}$ be arbitrary points in the complex $x$-plane and in the domain $D^{*}$ respectively. We choose a value of the integration constant $C_{1}$ so that the first relation of (5.3) (or $\left(5.3^{\prime}\right)$ ) holds at $x=x_{0}$ and $w=w^{0}$. Then, if $w$ tends to 0 along a curve $\Gamma_{w 0}^{*}\left(\right.$ contained in $\left.D^{*}\right)$ which starts from $w=w^{0}$ and approaches $w=0$ from the interior of the sector $D$, the point $x=\tilde{x}(w)$, where $\bar{x}(w)$ is defined by the first relation of (5.3) (or (5.3')), describes a spiral-shaped curve $\Gamma_{x_{0}}$ turning around the origin in the complex $x$-plane and approaches $x=0 . \quad$ The converse is also true.

\section{Proof of Theorem 2.1.}

First we consider Case A. Differentiating both sides of the relation (5.1) and substituting the expressions of the righthand members of (2.3) for $u^{\prime}$ and $v^{\prime}$ appearing in the resulting relation, it is found that

$$
x \frac{d w}{d x}=w^{2}\left(m \alpha+n \beta+\left(m \alpha^{\prime}+n \beta^{\prime}\right) w\right) .
$$


From this follows immediately the first equation of (5.2).

On the other hand, by (5.1), (2.3) is reduced to

$$
x u^{\prime}=u w\left(\alpha+\alpha^{\prime} w\right), \quad x v^{\prime}=v w\left(\beta+\beta^{\prime} w\right) .
$$

From this, we obtain the last two equations of (5.2) with the help of (6.1).

In Case $B$, it is easily verified that the reduced system $\left(2.3^{\prime}\right)$ admits an integral of the form

$$
C_{2}=u^{\nu} v^{-\mu} .
$$

Hence, the reduced system $\left(2.3^{\prime}\right)$ can be written as

$$
\left\{\begin{array}{l}
x u^{\prime}=\mu u^{m+1} v^{n}\left(\gamma+\gamma^{\prime} u^{m} v^{n}\right), \\
x v^{\prime}=\nu u^{m} v^{n+1}\left(\gamma+\gamma^{\prime} u^{m} v^{n}\right),
\end{array}\right.
$$

where

$$
\gamma^{\prime}=\sum \gamma_{h} C_{2}^{h}=\sum \gamma_{h}\left(u^{\nu} v^{-\mu}\right)^{h} .
$$

This system has the same form as (2.3) if we put

$$
\begin{cases}\alpha=\gamma \mu, & \alpha^{\prime}=\mu \gamma^{\prime}, \\ \beta=\gamma \nu, & \beta^{\prime}=\nu \gamma^{\prime} .\end{cases}
$$

Therefore, by the same reasoning as before, the reduced system $\left(2.3^{\prime}\right)$ is transformed into a system of the form (5.2') by introducing a new variable $w$ by (5.1) .

Thus the first part of Theorem 2.1 has been proved.

The remaining part of Theorem 2.1 can be easily proved by integrating the differential equations (5.2) (and (5.2')) by quadratures, and noticing that $u^{m} v^{n}=w$.

\section{Proof of Theorem 2.2.}

Following M. Hukuhara [1], we put

$$
\left\{\begin{array}{l}
x=C \exp \left(-\frac{m \alpha^{\prime}+n \beta^{\prime}}{(m \alpha+n \beta)^{2}} X\right), \\
w=\frac{m \alpha+n \beta}{\left(m \alpha^{\prime}+n \beta^{\prime}\right) Y} .
\end{array}\right.
$$


Then, as will be easily seen, the first equation of (5.2) (or the equation (6.1)) is transformed into an equation of the form

$$
\frac{d Y}{d X}=1+\frac{1}{Y} \text {. }
$$

M. Hukuhara [1] has shown that the general solution of this equation can be written as

$$
Y=\mathfrak{S}\left(X+C_{1}^{0}\right) \text {. }
$$

We consider the branch of $\mathfrak{S}(X)$ defined in Theorem 2.2. Therefore, the general solution of (6.1) is given by

$$
w=\frac{m \alpha+n \beta}{m \alpha^{\prime}+n \beta^{\prime}}\left(\mathfrak{s}\left(-\frac{(m \alpha+n \beta)^{2}}{m \alpha^{\prime}+n \beta^{\prime}} \log x+C_{1}^{0}\right)\right)^{-1} .
$$

It is to be noticed that the first equation of (5.2) is equivalent to the equation (6.1) and, consequently, the inverse function of $\tilde{x}(w)$ defined by the first relation of (5.3) coincides with the expression (7.4) if the arbitrary constant $C_{1}^{0}$ is suitably chosen.

Substituting (7.4) for $w$ into the last two relations of (5.3), we obtain the relations of the form (5.6) (which is the general solution of the reduced system (2.3)).

In Case $B$, we put

$$
x=C \exp \left(-\frac{\gamma^{\prime}}{\gamma^{2}(m \mu+n \nu)} X\right), \quad w=\frac{\gamma}{\gamma^{\prime} Y} .
$$

Then it is easy to see that the first equation of (5.2) is reduced to an equation of the same form as (7.2). Hence, an inverse function of $\bar{x}(w)$ defined by the first relation of $\left(5.3^{\prime}\right)$ can be written as

$$
\begin{gathered}
w=\frac{\gamma}{\gamma^{\prime} \mathcal{E}\left(X+C_{1}^{0}\right)}, \\
\left(X=-\frac{\gamma^{2}(m \mu+n \nu)}{\gamma^{\prime}} \log x\right)
\end{gathered}
$$

for a suitably chosen constant $C_{1}^{0}$. If we put this expression into the last two equations of $\left(5.3^{\prime}\right)$, we obtain the equations 
(5. $\left.6^{\prime}\right)$. This proves Theorem 2.2.

\section{Proof of Theorem 2.3.}

By the definition of the quantities $\theta_{+}$and $\theta_{-}$we have the inequalities

$$
\operatorname{Re} \frac{1}{(m \alpha+n \beta) w}>0, \quad \operatorname{Re} \frac{1}{\gamma\left(m_{\mu}+n \nu\right) w}>0
$$

for

$$
\theta_{-}<\arg w<\theta_{+}
$$

and the inequalities

$$
\operatorname{Re} \frac{1}{(m \alpha+n \beta) w} \leqq 0, \quad \operatorname{Re} \frac{1}{\gamma(m \mu+n \nu) w} \leqq 0
$$

for

$$
\theta_{-}-\pi<\arg w \leqq \theta_{-} \quad \text { or } \quad \theta_{+} \leqq \arg w<\theta_{+}+\pi \text {. }
$$

Clearly the domain $D$ (given by (5.7)) is contained in the sector (8.2). Hence, as $w$ tends to 0 in the sector $D$, the function $\tilde{x}(w)$ defined by the first relation of (5.3) (or 5.3')) vanishes exponentially. From this we can obtain Theorem 2.3 since we have

$$
\begin{array}{rlr}
\log \bar{x}(w) & =\log |\tilde{x}(w)|+i \arg \tilde{x}(w) \\
& = \begin{cases}-\frac{1}{(m \alpha+n \beta) w}+o(1), & \text { Case A } \\
-\frac{1}{\gamma(m \mu+n \nu) w}+o(1), & \text { Case B }\end{cases}
\end{array}
$$

Chapter III. Estimation of the growth of the general solution of the reduced system near the origin.

9. Properties of the solution of the reduced system (2.3) and $\left(2.3^{\prime}\right)$.

Let $u=U\left(x, x_{0}, u^{0}, v^{0}\right)$ and $v=V\left(x, x_{0}, u^{0}, v^{0}\right)$ be the holomorphic solution of the reudced system (2.3) or (2. $\left.3^{\prime}\right)$ satisfying 
the initial condition

$$
u^{0}=U\left(x_{0}, x_{0}, u^{0}, v^{0}\right), \quad v^{0}=V\left(x_{0}, x_{0}, u^{0}, v^{0}\right) .
$$

Let $\mathfrak{D}$ be an angular region

$$
\Theta_{-}<\arg w<\Theta_{+}, \quad 0<|w|<d\left(\arg w, c_{0}\right),
$$

where $d\left(\varphi, c_{0}\right)$ is a positive valued continuous function of both variables which will be defined later, and $\Theta_{-}$and $\Theta_{+}$are given by

$$
\left\{\begin{array}{l}
\Theta_{-}=-\pi-\min (\arg \alpha, \arg \beta)+4 \delta_{0}, \\
\Theta_{+}=\pi-\max (\arg \alpha, \arg \beta)-4 \delta_{0},
\end{array}\right.
$$

or

$$
\left\{\begin{array}{l}
\Theta_{-}=-\pi-\arg \gamma+3 \delta_{0}, \\
\Theta_{+}=\pi-\arg \gamma-3 \delta_{0},
\end{array}\right.
$$

according as we have Case $\mathrm{A}$ or Case $\mathrm{B}, \delta_{0}$ being an arbitrarily fixed sufficiently small positive constant.

Since the inequalities (1.4) are supposed to be satisfied, we can assume without loss of generality that

$$
|\arg \mathfrak{X}| \leqq \frac{\pi}{2}-3 \delta_{0}, \quad|\arg \mathfrak{B}| \leqq \frac{\pi}{2}-3 \delta_{0}
$$

for the same $\delta_{0}$ as before (see (5.4)).

Then we can assert that the angular region

$$
\Theta_{-}<\arg w<\Theta_{+}
$$

contains the sector

$$
\theta_{-}+2 \delta_{0} \leqq \arg w \leqq \theta_{+}-2 \delta_{0}
$$

with

$$
\theta_{ \pm}=-\arg (m \alpha+n \beta) \pm \frac{\pi}{2} \quad \text { for Case } \mathrm{A}
$$

or

$$
\theta_{ \pm}=-\arg \gamma \pm \frac{\pi}{2} \quad \text { for Case B }
$$

Indeed, for Case A, a direct calculation shows that 
$\Theta_{+}-\left(\theta_{+}-2 \delta_{0}\right)=\frac{\pi}{2}-\max (\arg \mathfrak{A}, \arg \mathfrak{B})-2 \delta_{0} \geqq \delta_{0} \quad($ by $(9.3))$

and

$$
\left(\theta_{-}+2 \delta_{0}\right)-\Theta_{-} \geqq \delta_{0}
$$

For Case B, from $\left(9.2^{\prime}\right)$ and $\left(9.4^{\prime}\right)$, we have

$$
\Theta_{+}-\left(\theta_{+}-2 \delta_{0}\right)=\left(\theta_{-}+2 \delta_{0}\right)-\Theta_{-}=\frac{\pi}{2}-\delta_{0} .
$$

The central angle of the angular domain $\mathfrak{D}$ is almost equal to

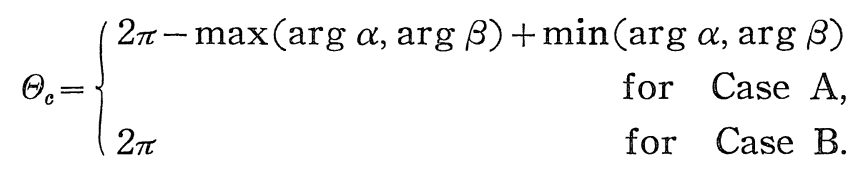

The aim of this chapter is to prove the following:

Theorem 3. There exists a spiral-shaped path $\Gamma_{x_{0}}$, winding around the origin of the complex $x$-plane, which starts from $x=x_{0}$ and approaches $x=0$, such that, when $x$ moves on this path, the value of $\left(x, U\left(x, x_{0}, u^{0}, v^{0}\right), V\left(x, x_{0}, u^{0}, v^{0}\right)\right)$ always remains in a domain of the form

$$
|x|<a_{0}, \quad|U|<b_{0}, \quad|V|<b_{0}, \quad U^{m} V^{n} \in \mathfrak{D}
$$

if $\left(x_{0}, u^{0}, v^{0}\right)$ satisfies the same condition.

Of course, the quantity $\delta_{0}$ appearing in (9.2) and (9.2') may depend on the choice of the values of $a_{0}, b_{0}$ and $\max _{\varphi} d\left(\varphi, c_{0}\right)$.

In the case when one of the quantities $m$ and $n$ is zero, as will be seen from the proof of Theorem 3, we have the following :

Theorem 3'. In the case when $n$ is zero, the conclusion of Theorem 3 is still valid if we replace the domain (9.6) by

$$
|x|<a_{0}, \quad|V|<b_{0}, \quad U^{m} \in \mathfrak{D},
$$

where $D$ is an angular region of the form

$$
\Theta_{-}<\arg w<\Theta_{+}, \quad 0<|w|<d\left(\arg w, c_{0}\right)
$$




$$
\Theta_{ \pm}= \pm \pi-\arg \beta \mp 4 \delta_{0} .
$$

In the case when $m$ is zero, the domain (9.7) must be replaced by

$$
|x|<a_{0}, \quad|U|<b_{0}, \quad V^{n} \in \mathfrak{D},
$$

where the angles $\Theta_{ \pm}$must be given by

$$
\Theta_{ \pm}= \pm \pi-\arg \alpha \mp 4 \delta_{0} .
$$

10. Determination of the path $\Gamma_{x_{0}}$.

We consider only the case when $m$ and $n$ are both positive. In the case when one of them is zero, the discussion can be carried out in quite a similar way. Instead of defining the path $\Gamma_{x_{0}}$ directly, we construct a path $\Gamma_{w_{0}}^{*}$ contained in $\mathfrak{D}$.

\section{$1^{\circ}$. Determination of the domain $\mathfrak{D}$.}

Let $A(\varphi)$ be a piecewise continuous function defined in the interval

$$
\Theta_{-} \leqq \varphi \leqq \Theta_{+}
$$

and satisfying an inequality

$$
\delta_{0} \leqq A(\varphi) \leqq \pi-\delta_{0} .
$$

Then the function $d\left(\varphi, c_{0}\right)$ is defined to be

$$
d\left(\varphi, c_{0}\right)=c_{0} \exp \int_{\theta_{0}}^{\varphi} \cot A(\varphi) d \varphi \quad\left(\Theta_{-} \leqq \theta_{0} \leqq \Theta_{+}\right),
$$

where $c_{0}$ is supposed to be sufficiently small.

Thus the angular region $\mathfrak{D}$ is the set of points $w=\rho e^{i \varphi}$ satisfying the inequalities

$$
\left\{\begin{array}{c}
0<\rho<c_{0} \exp \int_{\theta_{0}}^{\varphi} \cot A(\varphi) d \varphi, \\
\Theta_{-}<\varphi<\Theta_{+},
\end{array}\right.
$$

where $c_{0} e^{i \theta_{0}}$ is evidently a boundary point of $\mathfrak{D}$. The detailed definition of $A(\varphi)$ will be given by the formula (12.24). 
$2^{\circ}$. Determination of an auxiliary path $\Gamma_{w^{0}}^{*}$.

We put

$$
w^{0}=\left(u^{0}\right)^{m}\left(v^{0}\right)^{n},
$$

where $\left(x_{0}, u^{0}, v^{0}\right)$ is an arbitrarily chosen point in the domain (9. 6).

Let $(r, \theta)$ and $(\rho, \varphi)$ be the polar coordinates of the point $w^{0}$ and the variable point $w$ on the path $\Gamma_{w^{0}}^{*}$, and let $\theta_{ \pm}$be the angles given by (9.4) or (9. $\left.4^{\prime}\right)$.

Then the path $\Gamma_{w^{0}}^{*}$ will be defined as follows:

(i) The case when $\theta_{+}-2 \delta_{0}<\theta$. The path $\Gamma_{w^{0}}^{*}$ consists of a curvilinear part $\Gamma^{\prime}$ :

$$
\rho=r \exp \int_{\theta}^{\varphi} \cot A(\varphi) d \varphi, \quad \theta_{+}-2 \delta_{0} \leqq \varphi \leqq \theta
$$

and of a rectilinear part $\Gamma^{\prime \prime}$ :

$$
0<\rho \leqq r \exp \int_{\theta}^{\theta_{+}-2 \delta_{0}} \cot A(\varphi) d \varphi, \quad \varphi=\theta_{+}-2 \delta_{0} .
$$

(ii) The case when $\theta_{-}+2 \delta_{0} \leqq \theta \leqq \theta_{+}-2 \delta_{0}$. The path $\Gamma_{w^{0}}^{*}$ consists of only a rectilinear part $\Gamma^{\prime \prime}$ :

$$
0<\rho \leqq r, \quad \varphi=\theta .
$$

(iii) The case when $\theta<\theta_{-}+2 \delta_{0} . \quad$ The path $\Gamma_{w^{0}}^{*}$ consists of a curvilinear part $\Gamma^{\prime}$ :

$$
\rho=r \exp \int_{\theta}^{\varphi} \cot A(\varphi) d \varphi, \quad \theta \leqq \phi \leqq \theta_{-}+2 \delta_{0}
$$

and of a rectilinear part $\Gamma^{\prime \prime}$ :

$$
0<\rho \leqq r \exp \int_{\theta}^{\theta_{-}+2 \delta_{0}} \cot A(\varphi) d \varphi, \quad \varphi=\theta_{-}+2 \delta_{0} .
$$

\section{$3^{\circ}$. Determination of a path $\Gamma_{x_{0}}$.}

We denote by $\Gamma_{x_{0}}$ a curve in the complex $x$-plane obtained by mapping the curve $\Gamma_{w^{0}}^{*}$ by the first relation of (5.3) or $\left(5.3^{\prime}\right)$, where the integration constant $C_{1}$ must be determined so that 
$w=w^{0}$ is mapped into $x=x_{0}$.

Clearly, the path $\Gamma_{w^{0}}^{*}$ defined in $2^{\circ}$ is contained in the domain $\mathfrak{D}$ and, moreover, this path belongs to classes of the paths appearing in Theorem 2. 3 in 5. Hence $\Gamma_{x_{0}}$ describes a spiral-shaped path, turning around the origin in the complex $x$-plane, which starts from $x=x_{0}$ and approaches $x=0$.

We shall show that this curve will serve as a required path $\Gamma x_{0}$.

\section{Fundamental lemmas.}

As we have already seen in Theorem 2.3 in 5 , the correspondence between the points on these two curves is one to one. Hence, the solution $(U, V)$ and the variable point $x$ on $\Gamma_{x_{0}}$ can be regarded as functions of $w$ on $\Gamma_{w^{0}}^{*}$. We denote them by $(\tilde{U}(w), \tilde{V}(w))$ and $\tilde{x}(w)$. It is clear that

$$
U\left(\tilde{x}(w), x_{0}, u^{0}, v^{0}\right) \equiv \tilde{U}(w), \quad V\left(\tilde{x}(w), x_{0}, u^{0}, v^{0}\right) \equiv \tilde{V}(w) .
$$

An explicit representation of these three functions is given by the formula (5.3) or (5.3') if the aribtrary constants $C_{1}$ and $C_{2}$ are suitably chosen. Moreover, as we have already seen in the remark following Theorem 2.1 in $\mathbf{5}$, the condition (10.4) implies that

$$
\tilde{U}(w)^{m} \tilde{V}(w)^{n} \equiv w
$$

Hence we have $U^{n} V^{n}=w$ identically for $x \in \Gamma x_{0}$ (where $U, V$ and $w$ must be considered as functions of $x$.)

This proves the following:

Lemma 3.1. The value of the function $U^{m} V^{n}$ remains in the domain $(D)$ when $x$ moves on the path $\Gamma_{x_{0}}$.

In order to obtain Theorem 3, it is sufficient to prove the following :

Lemma 3.2. Let $s$ be the length of the curve $\Gamma_{w^{0}}^{*}$ measured from the origin to the point $w$. Then a function $A(\varphi)$, satisfying the conditions already given can be so chosen that, on the path 
$\Gamma_{w^{0}}^{*}$, we have inequalities of the form

$$
\begin{aligned}
& \frac{d|\tilde{x}|}{d s} \geqq \frac{\sin \delta_{0}}{2|m \alpha+n \beta|} \frac{|\tilde{x}|}{\rho^{2}}, \\
& \frac{d|\tilde{U}|}{d s} \geqq \frac{|\alpha| \sin \delta_{0}}{2|m \alpha+n \beta|} \frac{|\tilde{U}|}{\rho}, \\
& \frac{d|\tilde{V}|}{d s} \geqq \frac{|\beta| \sin \delta_{0}}{2|m \alpha+n \beta|} \frac{|\tilde{V}|}{\rho},
\end{aligned}
$$

or

$$
\begin{aligned}
& \frac{d|\tilde{x}|}{d s} \geqq \frac{\sin \delta_{0}}{2|\gamma|(m \mu+n \nu)} \frac{|\tilde{x}|}{\rho^{2}}, \\
& \frac{d|\tilde{U}|}{d s} \geqq \frac{\mu \sin \delta_{0}}{m \mu+n \nu} \frac{|\tilde{U}|}{\rho}, \\
& \frac{d|\tilde{V}|}{d s} \geqq \frac{\nu \sin \delta_{0}}{m_{\mu}+n \nu} \frac{|\tilde{V}|}{\rho},
\end{aligned}
$$

according as we have Case A or Case $\mathrm{B}$.

Indeed, these inequalities show us that, when $w$ moves from the origin to the point $w^{0}$ along the path $\Gamma_{w^{0}}^{*}$, the functions $\tilde{x}$, $\tilde{U}$ and $\tilde{V}$ are all monotonically increasing. From this it follows at once that

$$
|\tilde{x}| \leqq\left|x_{0}\right|, \quad|\tilde{U}| \leqq\left|u^{0}\right|, \quad|\tilde{V}| \leqq\left|v^{0}\right| .
$$

Therefore, we see by combining this with Lemma 3. 1 that, when $x$ moves on the path $\Gamma_{x_{0}},(x, U, V)$ always remains in the domain (9.6).

\section{Verificiaton of Lemma 3.2, Case A.}

It should be noticed that $(\rho, \varphi)$ and $(r, \theta)$ are respectively the polar coordinates of the variable point $w$ on the path $\Gamma_{w_{0}}^{*}$ and of the point $w^{0}$.

From the differential equations (5.2) we see that the derivatives of $|\tilde{x}|,|\tilde{U}|$ and $|\tilde{V}|$ with respect to $s$ are given by

$$
\frac{1}{|\tilde{x}|} \frac{d|\tilde{x}|}{d s}=\operatorname{Re}\left(\frac{1}{\tilde{x}} \frac{d \tilde{x}}{d s}\right)=\operatorname{Re}\left(\frac{1}{\tilde{x}} \frac{d \tilde{x}}{d w} \frac{d w}{d s}\right)
$$




$$
=\operatorname{Re}\left(\frac{1}{m \alpha+n \beta+\left(m \alpha^{\prime}+n \beta^{\prime}\right) w} \frac{1}{w^{2}} \frac{d w}{d s}\right),
$$

$$
\begin{aligned}
\frac{1}{|\tilde{U}|} \frac{d|\tilde{U}|}{d s} & =\operatorname{Re}\left(\frac{1}{\tilde{U}} \frac{d \tilde{U}}{d w} \frac{d w}{d s}\right) \\
& =\operatorname{Re}\left(\frac{\alpha+\alpha^{\prime} w}{m \alpha+n \beta+\left(m \alpha^{\prime}+n \beta^{\prime}\right) w} \frac{1}{w} \frac{d w}{d s}\right) \\
\frac{1}{|\tilde{V}|} \frac{d|\tilde{V}|}{d s} & =\operatorname{Re}\left(\frac{1}{\tilde{V}} \frac{d \tilde{V}}{d w} \frac{d w}{d s}\right) \\
& =\operatorname{Re}\left(\frac{\beta+\beta^{\prime} w}{m \alpha+n \beta+\left(m \alpha^{\prime}+n \beta^{\prime}\right) w} \frac{1}{w} \frac{d w}{d s}\right) .
\end{aligned}
$$

I. The rectilinear part $\Gamma^{\prime \prime}$.

In this case we have $s=\rho=|w|$ and $\phi=\arg w$. Moreover, $\phi$ satisfies the inequalities

$$
\theta_{-}+2 \delta_{0} \leqq \varphi \leqq \theta_{+}-2 \delta_{0}
$$

where $\theta_{+}$and $\theta_{-}$are given by (9.4). It follows immediately that

$$
|\varphi+\arg (m \alpha+n \beta)| \leqq \frac{\pi}{2}-2 \delta_{0}
$$

and

$$
\frac{d w}{d s}=e^{i \varphi}
$$

Hence (12.1), (12.2) and (12.3) respectively imply that:

$$
\frac{1}{|\bar{x}|} \frac{d|\tilde{x}|}{d s}=\frac{1}{\rho^{2}} \operatorname{Re}\left(\frac{e^{-i \varphi}}{m \alpha+n \beta}\left(1+\frac{m \alpha^{\prime}+n \beta^{\prime}}{m \alpha+n \beta} w\right)^{-1}\right)
$$

$$
\begin{aligned}
& \frac{1}{|\tilde{U}|} \frac{d|\tilde{U}|}{d s}=\frac{1}{\rho} \operatorname{Re}\left(\frac{\alpha}{m \alpha+n \beta}\left(1+\frac{\alpha^{\prime}}{\alpha} w\right)\left(1+\frac{m \alpha^{\prime}+n \beta^{\prime}}{m \alpha+n \beta} w\right)^{-1}\right) \\
& \frac{1}{|\tilde{V}|} \frac{d|\tilde{V}|}{d s}=\frac{1}{\rho} \operatorname{Re}\left(\frac{\beta}{m \alpha+n \beta}\left(1+\frac{\beta^{\prime}}{\beta} w\right)\left(1+\frac{m \alpha^{\prime}+n \beta^{\prime}}{m \alpha+n \beta} w\right)^{-1}\right)
\end{aligned}
$$

Since $|w|$ is supposed to be sufficiently small, it does not lose any generality to assume that: 
(12.9)

(12. 10)

(12. 11)

$$
\begin{aligned}
& \left\{\begin{array}{l}
\left|1+\frac{m \alpha^{\prime}+n \beta^{\prime}}{m \alpha+n \beta} w\right|<2, \\
\left|\arg \left(1+\frac{m \alpha^{\prime}+n \beta^{\prime}}{m \alpha+n \beta} w\right)\right|<\delta_{0},
\end{array}\right. \\
& \left\{\begin{array}{l}
\left|\left(1+\frac{\alpha^{\prime}}{\alpha} w\right)\left(1+\frac{m \alpha^{\prime}+n \beta^{\prime}}{m \alpha+n \beta} w\right)^{-1}\right|>\frac{1}{2}, \\
\left|\arg \left(1+\frac{\alpha^{\prime}}{\alpha} w\right)\left(1+\frac{m \alpha^{\prime}+n \beta^{\prime}}{m \alpha+n \beta} w\right)^{-1}\right|<\delta_{0},
\end{array}\right. \\
& \left\{\begin{array}{l}
\left|\left(1+\frac{\beta^{\prime}}{\beta} w\right)\left(1+\frac{m \alpha^{\prime}+n \beta^{\prime}}{m \alpha+n \beta} w\right)^{-1}\right|>\frac{1}{2}, \\
\left|\arg \left(1+\frac{\beta^{\prime}}{\beta} w\right)\left(1+\frac{m \alpha^{\prime}+n \beta^{\prime}}{m \alpha+n \beta} w\right)^{-1}\right|<\delta_{0} .
\end{array}\right.
\end{aligned}
$$

From (12.9) and (12.4) we can easily derive the inequality (11.1). Noticing that the inequalities (9.3) are satisfied, we see that the inequalities (11.2) and (11.3) are an immediate consequence of (12.7), (12.10) and (12.8), (12.11) respectively. Thus Lemma 3.2 has been established on the rectilinear part $\Gamma^{\prime \prime}$.

\section{The curvilinear part $\Gamma^{\prime}$.}

In this case, $\rho$ is a function of $\phi$ given by (10.5) or (10.8) according as $\theta_{+}-2 \delta_{0}<\theta$ or $\theta<\theta_{-}+2 \delta_{0}$. Hence we have

$$
\frac{d w}{d \varphi}=w(\cot A(\varphi)+i)
$$

and

$$
d s= \pm \frac{\rho}{\sin A(\varphi)} d \varphi
$$

according as $\theta_{+}-2 \delta_{0}<\theta$ or $\theta<\theta_{-}+2 \delta_{0}$. From this it follows immediately that

$$
\frac{d w}{d s}= \pm e^{(A(\varphi)+\varphi) i}
$$

according as $\theta_{+}-2 \delta_{0}<\theta$ or $\theta<\theta_{-}+2 \delta_{0}$.

(i) Case when $\theta_{+}-2 \delta_{0}<\theta$. 
Identities (12.1), (12.2) and (12.3) respectively imply that:

$$
\begin{aligned}
& |\tilde{x}|^{-1} \frac{d|\tilde{x}|}{d s}=\frac{1}{\rho^{2}} \operatorname{Re}\left(\frac{e^{(A(\varphi)-\varphi) i}}{m \alpha+n \beta}\left(1+\frac{m \alpha^{\prime}+n \beta^{\prime}}{m \alpha+n \beta} w\right)^{-1}\right) \\
& |\check{U}|^{-1} \frac{d|\tilde{U}|}{d s}=\frac{1}{\rho} \operatorname{Re}\left(\frac{\alpha e^{A(\varphi) i}}{m \alpha+n \beta}\left(1+\frac{\alpha^{\prime}}{\alpha} w\right)\left(1+\frac{m \alpha^{\prime}+n \beta^{\prime}}{m \alpha+n \beta} w\right)^{-1}\right) \\
& |\tilde{V}|^{-1} \frac{d|\tilde{V}|}{d s}=\frac{1}{\rho} \operatorname{Re}\left(\frac{\beta e^{A(\varphi) i}}{m \alpha+n \beta}\left(1+\frac{\beta^{\prime}}{\beta} w\right)\left(1+\frac{m \alpha^{\prime}+n \beta^{\prime}}{m \alpha+n \beta} w\right)^{-1}\right)
\end{aligned}
$$

By virtue of the inequalities (12.9), (12.10) and (12.11) we see that the inequalities (11.1), (11.2) and (11.3) follow respectively from the inequalities:

$$
\begin{aligned}
& \text { (12.13) }-\frac{\pi}{2}+\delta_{0} \leqq A(\varphi)-\varphi-\arg (m \alpha+n \beta) \pm \delta_{0} \leqq \frac{\pi}{2}-\delta_{0}, \\
& (12.14) \quad-\frac{\pi}{2}+\delta_{0} \leqq A(\varphi)+\arg \mathfrak{A} \pm \delta_{0} \leqq \frac{\pi}{2}-\delta_{0}, \\
& \text { (12.15) } \quad-\frac{\pi}{2}+\delta_{0} \leqq A(\varphi)+\arg \mathfrak{S} \pm \delta_{0} \leqq \frac{\pi}{2}-\delta_{0}, \\
& \text { for } \theta_{+}-2 \delta_{0} \leqq \varphi \leqq \Theta_{+} .
\end{aligned}
$$

Therefore, observing that $\delta_{0} \leqq A(\varphi) \leqq \pi-\delta_{0}$, we see that the condition which $A(\varphi)$ must satisfy is written as

$$
\begin{aligned}
\max \{\max (\varphi+\arg (m \alpha+n \beta),-\arg \mathfrak{A},-\arg \mathfrak{B}) \\
\left.\quad-\frac{\pi}{2}+2 \delta_{0}, \delta_{0}\right\} \leqq A(\varphi) \\
\leqq \\
\min \{\min (\varphi+\arg (m \alpha+n \beta),-\arg \mathfrak{A},-\arg \mathfrak{B}) \\
\left.+\frac{\pi}{2}-2 \delta_{0}, \pi-\delta_{0}\right\} .
\end{aligned}
$$

However, the inequalities (9.3) imply that

$$
\begin{gathered}
\max \left\{-\min (\arg \mathfrak{A}, \arg \mathfrak{B})-\frac{\pi}{2}+2 \delta_{0}, \delta_{0}\right\}=\delta_{0}, \\
\min \left\{-\max (\arg \mathfrak{A}, \arg \mathfrak{B})+\frac{\pi}{2}-2 \delta_{0}, \pi-\delta_{0}\right\} \\
=-\max (\arg \mathfrak{A}, \arg \mathfrak{B})+\frac{\pi}{2}-2 \delta_{0},
\end{gathered}
$$


and the definition of $\theta_{+}$and $\theta_{-}$yields

$$
-\arg (m \alpha+n \beta) \pm \frac{\pi}{2}=\theta_{ \pm} .
$$

Hence, the inequality (12.16) from which the symbol $\leqq A(\varphi)$ was removed can be written in the form

$$
\begin{array}{r}
\max \left(\varphi-\theta_{+}+2 \delta_{0}, \delta_{0}\right) \leqq \min \left(\rho-\theta_{-}-2 \delta_{0},\right. \\
\left.-\max (\arg \mathfrak{A}, \arg \mathfrak{B})+\frac{\pi}{2}-2 \delta_{0}\right) .
\end{array}
$$

This is equivalent to

$$
\varphi-\theta_{+}+2 \delta_{0} \leqq \varphi-\theta_{-}-2 \delta_{0} ;
$$

$$
\varphi-\theta_{+}+2 \delta_{0} \leqq-\max (\arg \mathfrak{A}, \arg \mathfrak{B})+\frac{\pi}{2}-2 \delta_{0} ;
$$

$$
\delta_{0} \leqq \varphi-\theta_{-}-2 \delta_{0} ;
$$

$$
\delta_{0} \leqq-\max (\arg \mathfrak{A}, \arg \mathfrak{B})+\frac{\pi}{2}-2 \delta_{0} .
$$

The inequality (12.17.1) is automatically satisfied since $\theta_{-}<\theta_{+}$and $\delta_{0}$ is supposed to be suffiiciently small. The inequality (12.17.4) is an immediate consequence of (9.3). Therefore, it is found that the inequality (12.17) is satisfied for

$$
\theta_{-}+3 \delta_{0} \leqq \varphi \leqq \theta_{+}+\frac{\pi}{2}-\max (\arg \mathfrak{A}, \arg \mathfrak{B})-4 \delta_{0} .
$$

However, the last expression of the inequality is equal to the quantity $\Theta_{+}$(see (9.2)) and the interval (12.18) contains

$$
\theta_{+}-2 \delta_{0} \leqq \varphi \leqq \Theta_{+} .
$$

Consequently, the inequality (12.16) is certainly satisfied in the interval in question if we put, for example,

$$
A(\varphi)=\max \left(\varphi-\theta_{+}+2 \delta_{0}, \delta_{0}\right) \quad \text { for } \varphi \epsilon\left[\theta_{+}-2 \delta_{0}, \Theta_{+}\right] .
$$

Remark. If $n=0$ (or $m=0$ ), the condition (12.14) (or (12.15)) is unnecessary. 
(ii) Case when $\theta<\theta_{-}+2 \delta_{0}$.

By the same reasoning as before we can prove that the inequalities (11.1), (11.2) and (11.3) are simultaneously satisfied if the function $A(\varphi)$ satisfies an inequality of the form

$$
\begin{aligned}
\max \{\max (\varphi+\arg (m \alpha+n \beta),-\arg \mathfrak{A},-\arg \mathfrak{B}) \\
\left.+\frac{\pi}{2}+2 \delta_{0}, \delta_{0}\right\} \leqq A(\varphi) \\
\leqq \min \{\min (\varphi+\arg (m \alpha+n \beta),-\arg \mathfrak{A},-\arg \mathfrak{S}) \\
\left.+\frac{3 \pi}{2}-2 \delta_{0}, \pi-\delta_{0}\right\} .
\end{aligned}
$$

Since, by (9.3), we have

$$
\begin{aligned}
& -\min (\arg \mathfrak{A}, \arg \mathfrak{B})+\frac{\pi}{2}+2 \delta_{0}>\delta_{0}, \\
& -\max (\arg \mathfrak{A}, \arg \mathfrak{B})+\frac{3 \pi}{2}-2 \delta_{0} \geqq \pi-\delta_{0},
\end{aligned}
$$

the inequality (12.20) from which $\leqq A(\phi)$ was removed will be reduced to

$$
\begin{aligned}
& \max \left(\varphi-\theta_{+}+\pi+2 \delta_{0},-\min (\arg \mathfrak{A}, \arg \mathfrak{S})+\frac{\pi}{2}+2 \delta_{0}\right) \\
& \leqq \min \left(\varphi-\theta_{-}+\pi-2 \delta_{0}, \pi-\delta_{0}\right) .
\end{aligned}
$$

By the same method as in the proof of the inequality (12.17), we can easily verify that this inequality holds in the interval

$$
\theta_{-}-\frac{\pi}{2}-\min (\arg \mathfrak{A}, \arg \mathfrak{B})+4 \delta_{0} \leqq \varphi \leqq \theta_{+}-3 \delta_{0} \text {. }
$$

The first expression is equal to the quantity $\Theta_{-}$, and this interval contains the interval $\left[\Theta_{-}, \theta_{-}+2 \delta_{0}\right]$ (See 9).

If we put, for example, (12. 23) $A(\varphi)=\min \left(\varphi-\theta_{-}+\pi+2 \delta_{0}, \pi-\delta_{0}\right) \quad$ for $\varphi \epsilon\left[\Theta_{-}, \theta_{-}+2 \delta_{0}\right]$, the inequality (12.20) is certainly satisfied in the interval in question.

Thus we have proved that, to obtain Lemma 3.2 for Case A, 
it is sufficient to put

$$
A(\varphi)= \begin{cases}\max \left(\varphi-\theta_{+}+2 \delta_{0}, \delta_{0}\right) & \text { for } \varphi \epsilon\left[\theta_{+}-2 \delta_{0}, \Theta_{+}\right], \\ \frac{\pi}{2} & \text { for } \varphi \epsilon\left[\theta_{-}+2 \delta_{0}, \theta_{+}-2 \delta_{0}\right], \\ \min \left(\varphi-\theta_{-}+\pi-2 \delta_{0}, \pi-\delta_{0}\right) & \text { for } \varphi \epsilon\left[\Theta_{-}, \theta_{-}+2 \delta_{0}\right],\end{cases}
$$

with

$$
\begin{gathered}
\theta_{ \pm}=-\arg (m \alpha+n \beta) \pm \frac{\pi}{2}, \\
\Theta_{-}=-\pi-\min (\arg \alpha, \arg \beta)+4 \delta_{0}, \\
\Theta_{+}=\pi-\max (\arg \alpha, \arg \beta)-4 \delta_{0} .
\end{gathered}
$$

\section{Verification of Lemma 3.2 for Case $\mathbb{B}$.}

In the differential equations (5. $\left.2^{\prime}\right)$, the following facts should be noticed :

(i) $\frac{\mu}{m \mu+n \nu}$ and $\frac{\nu}{m \mu+n \nu}$ are real positive numbers;

(ii) the expression $\gamma^{\prime} w$ is sufficiently small in absolute value provided that $|\check{U}|$ and $|\tilde{V}|$ are sufficiently small.

The assertion (ii) can be easily verified in the following way: Since we have

$$
\gamma^{\prime}=\sum_{h}\left(\tilde{U}^{\nu} \tilde{V}^{-\mu}\right)^{h} \cdot \gamma_{h}
$$

and the condition $\gamma_{h} \neq 0$ implies that $m+\nu h$ and $n-\mu h$ are nonnegative integers not simultaneously zero, we have

$$
\gamma^{\prime} w=\sum \gamma_{h}\left(\tilde{U}^{\nu} \tilde{U}^{-\mu}\right)^{h} \tilde{U}^{m} \tilde{V}^{n}=\sum \gamma_{h} \tilde{U}^{m+\nu h} \tilde{V}^{n-\mu h},
$$

if we notice that $w=\tilde{U}^{m} \tilde{V}^{n}$. From these relations we have (ii).

By virtue of (ii), we can suppose without loss of generality that we have inequalities

$$
\left|1+\frac{\gamma^{\prime}}{\gamma} w\right| \leqq 2, \quad\left|\arg \left(1+\frac{\gamma^{\prime}}{\gamma} w\right)\right| \leqq \delta_{0}
$$

for an arbitrarily fixed sufficiently small positive constant $\delta_{0}$.

We can now prove Lemma 3.2 without any essential modification of the proof for Case A. 
Indeed, on the rectilinear part $\Gamma^{\prime \prime}$, we can prove by means of (13.2) that the inequalities $\left(11.1^{\prime}\right),\left(11.2^{\prime}\right)$ and $\left(11.3^{\prime}\right)$ are satisfied. On the curvilinear part $\Gamma^{\prime}$, we can easily verify that the conditions which the function $A(\varphi)$ must satisfy are written as

$$
\begin{aligned}
& \max \left(\rho-\theta_{+}+2 \delta_{0}, \delta_{0}\right) \leqq A(\rho) \\
& \quad \leqq \min \left(\varphi-\theta_{-}-2 \delta_{0}, \frac{\pi}{2}-\delta_{0}\right) \quad \text { for } \theta_{+}-2 \delta_{0} \leqq \varphi \leqq \Theta_{+}
\end{aligned}
$$

and

$$
\begin{aligned}
\max ( & \left(\varphi-\theta_{+}+\pi+2 \delta_{0}, \frac{\pi}{2}+\delta_{0}\right) \leqq A(\varphi) \\
& \leqq \min \left(\varphi-\theta_{-}+\pi-2 \delta_{0}, \pi-\delta_{0}\right) \text { for } \Theta_{-} \leqq \varphi \leqq \theta_{-}+2 \delta_{0},
\end{aligned}
$$

where $\theta_{+}$and $\theta_{-}$are given by $\left(9.4^{\prime}\right)$, and that the inequalities (13.3) and (13.4) (from which the symbol $\leqq A(\varphi)$ was removed) are satisfied in the intervals

$$
\left[\theta_{-}+3 \delta_{0}, \Theta_{+}\right] \quad \text { and }\left[\Theta_{-}, \theta_{+}-3 \delta_{0}\right]
$$

respectively, where $\Theta_{+}$and $\Theta_{-}$are given by (9.2').

Clearly, these two intervals contain respectively the intervals $\left[\theta_{+}-2 \delta_{0}, \Theta_{+}\right]$and $\left[\Theta_{-}, \theta_{-}+2 \delta_{0}\right]$ in question (See 9). Hence, if we define the function $A(\varphi)$, for example, by a formula of the same form as (12.24) (where $\theta_{ \pm}$must be replaced by $-\arg \gamma \pm \frac{\pi}{2}$ respectively), the inequalities (13.3) and (13.4) are certainly satisfied in the intervals in question and, consequently, the inequalities $\left(11.1^{\prime}\right),\left(11.2^{\prime}\right)$ and $\left(11.3^{\prime}\right)$ are simultaneously satisfied on the curvilinear part. This proves Lemma 3.2 for Case B.

\section{Pare 1 . The formal solutions.}

This part will be devoted to the construction of formal solutions of diverse types. 
Chapter IV. Determination of formal solutions of the system (1.1).

\section{Formal solutions of diverse types.}

Let $A(\varphi), \Theta_{-}$and $\Theta_{+}$be the same as those that appeared in the preceding chapter. In the case when $m$ and $n$ are both positive, $\Theta_{ \pm}$are given by (9.2) or (9.2') according as we have Case A or Case B. While, in the case when one of $m$ and $n$ is zero, they are given by (9.9) or (9.11) according as we have $n=0$ or $m=0$.

We put

$$
d(\varphi, c)=c \exp \int_{\theta_{0}}^{\varphi} \cot A(\varphi) d \varphi, \quad \Theta_{-} \leqq \varphi \leqq \Theta_{+},
$$

where $\theta_{0}$ is contained in the interval $\left[\Theta_{-}, \Theta_{+}\right]$and $c$ is a sufficiently small positive constant, and define an angular region $\mathfrak{D}(c)$ by

$$
\mathfrak{D}(c)=\left\{w ; \Theta_{-}<\arg w<\Theta_{+}, \quad 0<|w|<d(\arg w, c)\right\} .
$$

Let $(U, V) \equiv\left(U\left(x, x_{0}, u^{0}, v^{0}\right), \quad V\left(x, x_{0}, u^{0}, v^{0}\right)\right)$ be the holomorphic solution of the reduced system (2.3) or (2.3') (according as we have Case A or Case B) such that $(u, v)=\left(u^{0}, v^{0}\right)$ at $x=x_{0}$, where $x_{0}, u^{0}, v^{0}$ are arbitrarily chosen such that

$$
\left|x_{0}\right|<a_{0}, \quad\left|u^{0}\right|<b_{0}, \quad\left|v^{0}\right|<b_{0}, \quad u^{0 m} v^{0^{n}} \in \mathfrak{D}\left(c_{0}\right) .
$$

If $n=0,(14.3)$ must be replaced by

$$
\left|x_{0}\right|<a_{0}, \quad\left|v^{0}\right|<b_{0}, \quad u^{0 m} \in \mathfrak{D}\left(c_{0}\right) .
$$

If $m=0,(14.3)$ must be replaced by

$$
\left|x_{0}\right|<a_{0}, \quad\left|u^{0}\right|<b_{0}, \quad v^{0 n} \in \mathfrak{D}\left(c_{0}\right) .
$$

Remark. The point $\left(u^{0}, v^{0}\right)$ satisfying the last three inequalities of (14.3) is located on the product space of the Riemann surfaces of $\log u$ and $\log v$.

By virtue of Theorem 1 in 2 , if we replace $(u, v)$ by $(U, V)$ we 
have immediately the following:

Theorem 4.1. The differential equations (1.1) admit formal solution that are triple power series of $x, U, V$ :

$$
y \sim U+\sum^{(2)} P_{j k l} x^{j} U^{k} V^{l}, \quad z \sim V+\sum^{(2)} Q_{j k l} x^{j} U^{k} V^{l},
$$

where

$$
P_{00 t}=0, \quad Q_{0 k 0}=0, \quad(l, k=1,2, \cdots) .
$$

To investigate the analytical meaning of this formal expression, we formally rewrite it in a form of double or single power series. First we rearrange it in a double power series form, in three different ways, as follows:

$$
\left\{\begin{array}{l}
y \sim U+\sum P_{h l}^{(1)}(x) U^{k} V^{\imath} \\
z \sim V+\sum Q_{h l}^{(1)}(x) U^{k} V^{\imath}
\end{array}\right.
$$

where

$$
\begin{gathered}
P_{k l}^{(1)}(x) \sim \sum_{j} P_{j k l} x^{j}, \quad Q_{k l}^{(1)}(x) \sim \sum_{j} Q_{j k l} x^{j} . \\
\left\{\begin{array}{l}
y \sim U\left(1+\sum_{l=0}^{\infty} P_{i}^{(2)}(U) V^{l}\right)+\sum_{j \geqq 1, l \geq 0} P_{j l}^{(2)}(U) x^{j} V^{l}, \\
z \sim V\left(1+\sum_{l=0}^{\infty} Q_{l}^{(2)}(U) V^{l}\right)+\sum_{j \geqq 1, l \geq 0} Q_{j l}^{(2)}(U) x^{j} V^{l},
\end{array}\right.
\end{gathered}
$$

where

$$
\begin{gathered}
P_{l}^{(2)}(u) \sim \sum_{k=1}^{\infty} P_{0 k l} u^{k-1}, \quad Q_{l}^{(2)}(u) \sim \sum_{k=0}^{\infty} Q_{0 k l+1} u^{k}, \\
P_{j l}^{(2)}(u) \sim \sum_{k} P_{j k l} u^{k}, \quad Q_{j l}^{(2)}(u) \sim{ }_{k} \sum Q_{j k l} u^{k} . \\
\left\{\begin{array}{l}
y \sim U\left(1+\sum_{k=0}^{\infty} P_{k}^{(3)}(V) U^{k}\right)+\sum_{j \geq 1, k \geq 0} P_{j k}^{(3)}(V) x^{j} U^{k}, \\
z \sim V\left(1+\sum_{k=0}^{\infty} Q_{k}^{(3)}(V) U^{k}\right)+\sum_{j \geq 1, k \geq 0} Q_{j k}^{(3)}(V) x^{j} U^{k},
\end{array}\right.
\end{gathered}
$$

where

$$
\begin{aligned}
P_{k}^{(3)}(v) \sim \sum_{l=0}^{\infty} P_{j k+1 l} v^{l}, & Q_{k}^{(3)}(v) \stackrel{\infty}{\sim} \sum_{l=1} Q_{j k l} v^{l-1}, \\
P_{j k}^{(3)}(v) \sim \sum_{l} P_{j k l} v^{l}, & Q_{j k}^{(3)}(v) \sim \sum_{l} Q_{j k l} v^{l} .
\end{aligned}
$$


It is evident that these expressions are also formal solutions. In order to give them an analytical meaning, it is first necessary that the coefficients of these double power series (which are also the formal power series of a single variable) must admit some analytical interpretation.

Concerning this, we have following three theorems.

Theorem 4.2.1. If we formally rewrite the formal solution (I) in the form (II. 1), the coefficients $\left(P_{k l}^{(1)}(x), Q_{k l}^{(1)}(x)\right)$ are functions holomorphic and bounded in $x$ for

$$
|x|<a_{0}^{\prime}
$$

so that the formal series (14.5) are uniformly convergent there. Consequently, the formal equality $\sim$ can be replaced by the true equality $=$ in (14.5).

Theorem 4.2.2. Let us formally rewrite the formal solution (I) in the form (II.2). Then, in the case when $n>0$, the coefficients $\left(P_{l}^{(2)}(u), Q_{l}^{(2)}(u)\right)$ and $\left(P_{j l}^{(2)}(u), Q_{j l}^{(2)}(u)\right)$ are all holomorphic and bounded functions of $u$ for

$$
|u|<b_{0}^{\prime}
$$

and, consequently, the formal power series (14.6) and (14.7) are uniformly convergent there.

In the case when $n=0,\left(P_{j l}^{(2)}(u), Q_{j l}^{(2)}(u)\right)$ for $j \geqq 1$ are functions holomorphic and bounded in $u$ for

$$
|m \arg u+\arg \alpha|<\frac{3 \pi}{2}, \quad 0<|u|<b_{0}^{\prime}
$$

and admit asymptotic expansions of the form (14.7) as $u$ tends to 0 in the domain (14.11').

Theorem 4.2 .3 . If we formally rearrange the formal solution (I) in the form (III. 3), the coefficients $\left(\mathbb{P}_{k}^{(3)}(v), Q_{k}^{(3)}(v)\right)$ and $\left(P_{j k}^{(3)}(v), Q_{j k}^{(3)}(v)\right)$ are functions holomorphic and bounded in $v$ for

$$
|v|<b_{0}^{\prime}
$$

and, consequently, the formal power series (14.8) and (14.9) are 
uniformly convergent there.

In the case when $m=0,\left(P_{j k}^{(3)}(v), Q_{j k}^{(3)}(v)\right)$ for $j \geqq 1$ are functions holomorphic and bounded in $v$ for

$$
|n \arg v+\arg \beta|<\frac{3 \pi}{2}, \quad 0<|v|<b_{0}^{\prime}
$$

and admitting asymptotic developements of the form (14.9) as $v$ tends to 0 in the domain (14.12').

Next we consider the rearrangement of (I) in a single power series form. This can also be done by rearranging the double power series expressions (II.1), (II.2) and (II.3) in a suitable way, and they are written, in three different ways, as follows :

$$
\left\{\begin{array}{l}
y \sim U\left(1+P_{0}^{(1)}(U, V)\right)+\sum_{j=1}^{\infty} P_{j}^{(1)}(U, V) x^{j} \\
z \sim V\left(1+Q_{0}^{(1)}(U, V)\right)+\sum_{j=1}^{\infty} Q_{j}^{(1)}(U, V) x^{j}
\end{array}\right.
$$

where

$$
\left\{\begin{array}{l}
P_{0}^{(1)}(u, v) \sim \sum_{k \geqq 1, l \geqq 0} P_{0 k l} u^{k-1} v^{l} \\
Q_{0}^{(1)}(u, v) \sim \sum_{h \geqq 0, l \leqq 1} Q_{0 k l} u^{k} v^{l-1}
\end{array}\right.
$$

$$
\begin{gathered}
P_{j}^{(1)}(u, v) \sim \sum_{k, l} P_{j k l} u^{k} v^{l}, \quad Q_{\jmath}^{(1)}(u, v) \sim \sum_{k, l} Q_{j k l} u^{k} v^{l} \\
y \sim \sum_{l=0}^{\infty} P_{l}^{(2)}(x, U) V^{l}, \quad z \sim \sum_{l=0}^{\infty} Q_{l}^{(2)}(x, U) V^{\imath}
\end{gathered}
$$

where, by (II. 2),

$$
\begin{aligned}
& \left\{\begin{array}{l}
P_{l}^{(2)}(x, u) \sim \delta_{l 0} u+u P_{l}^{(2)}(u)+\sum_{\jmath=1}^{\infty} P_{\jmath l}^{(2)}(u) x^{j}, \\
Q_{l}^{(2)}(x, u) \sim \delta_{l 1}+Q_{l-1}^{(2)}(u)+\sum_{\jmath=1}^{\infty} Q_{\jmath l}^{(2)}(u) x^{j}
\end{array}\right. \\
& y \sim \sum_{h=0}^{\infty} P_{h}^{(3)}(x, V) U^{k}, \quad z \sim \sum_{k=0}^{\infty} Q_{h}^{(3)}(x, V) U^{k},
\end{aligned}
$$

where, by (II. 3), 


$$
\left\{\begin{array}{l}
P_{h}^{(3)}(x, v) \sim \delta_{k 1}+P_{k-1}^{(3)}(v)+\sum_{j=1}^{\infty} P_{j k}^{(3)}(v) x^{j}, \\
Q_{k}^{(3)}(x, v) \sim \delta_{k 0} v+v Q_{k}^{(3)}(v)+\sum_{j=1}^{\infty} Q_{j k}^{(3)}(v) x^{j}
\end{array}\right.
$$

Here $\delta_{k l}$ is the Kronecker's notation. Evidently these formal expressions (III. 1), (III. 2) and (III.3) are also formal solutions.

As regards the analytical meaning of the coefficients of these formal solutions, we obtain the following three theorems:

Theorem 4. 3.1. If we rewrite the formal solution (I) in the form (III. 1), the coefficients $\left(P_{j}^{(1)}(U, V), Q_{j}^{(1)}(U, V)\right)(j=0,1, \cdots)$ can be uniquely determined as solutions of certain non-linear differential equations (for $j=0$ ) or of certain linear differential equations ( for $j \geqq 1$ ) such that:

The coefficients $\left(P_{0}^{(1)}(u, v), Q_{0}^{11}(u, v)\right)$ are functions holomorphic and bounded in $(u, v)$ for

$$
|u|<b_{0}^{\prime}, \quad|v|<b_{0}^{\prime}
$$

and, consequently, the formal power series (14.13) are uniformly convergent there.

The coefficients $P_{j}^{(1)}(u, v)$ and $Q_{j}^{(1)}(u, v)(j \geqq 1)$ are functions holomorphic and bounded in $(u, v)$ for

$$
|u|<b_{0}^{\prime}, \quad|v|<b_{0}^{\prime}, \quad u^{m} v^{n} \in \mathfrak{D}\left(c_{0}^{\prime}\right)
$$

admitting asymptotic expansions of the form (14.14) as $u$ and $v$ tend to 0 in the domain (14.18).

Remark. In the case when $m=0$, the functions $P_{j}^{(1)}(u, v)$ and $Q_{J}{ }^{1)}(u, v)$ are developable in uniformly convergent power series of $u$ in

$$
|u|<b_{0}^{\prime}, \quad v^{n} \in \mathcal{D}\left(c_{0}^{\prime}\right)
$$

such that the coefficients are functions asymptotically developable in powers of $v$ for

$$
\theta<|v|^{n}<c_{0}^{n}, \quad|n \arg v+\arg \beta|<\frac{3 \pi}{2} .
$$

By the inequalities (9.3), we see that the domain $\mathfrak{D}\left(c_{0}^{\prime}\right)$ 
appearing in (14.19) is contained in the domain (14.20) for a suitably chosen positive constant $c_{0}^{\prime}$.

In the case when $n=0$, the functions $P_{j}^{(1)}(u, v)$ and $Q_{j}^{\prime 1)}(u, v)$ are developable in uniformly convergent power series of $v$ in

$$
|v|<b_{0}^{\prime}, \quad u^{m} \equiv \mathfrak{D}\left(c_{0}^{\prime}\right)
$$

whose coefficients are functions admitting asymptotic expansions in powers of $u$ for

$$
0<|u|^{m}<c_{0}^{\prime}, \quad|m \arg u+\arg \alpha|<\frac{3}{2} \pi .
$$

By virtue of (9.3), the domain $\mathfrak{D}\left(c_{0}^{\prime}\right)$ is contained in the domain above if we choose suitably the positive constant $c_{0}^{\prime}$.

Theorem 4.3.2. If we rewrite the formal solution (II. 2) in the form (III. 2), the coefficients $\left(P_{l}^{(2)}(x, u), Q_{l}^{(2)}(x, u)\right)(l=0,1, \cdots)$ are functions holomorphic and bounded in $(x, u)$ for

$$
|x|<a_{0}^{\prime}, \quad|u|<b_{0}^{\prime}
$$

so that they can be developed there in uniformly convergent power series of $x$ and $u$. Therefore, the formal series (14.15) are naturally uniformly convergent.

In the case when $n=0, P_{l}^{2}(x, u)$ and $Q_{l}^{2}(x, u)$ are functions holomorphic and bounded in $(x, u)$ for

$$
|x|<a_{0}^{\prime} . \quad|m \arg u+\arg \alpha|<\frac{3 \pi}{2}, \quad 0<|u|<b_{0}^{\prime}
$$

and developable in the uniformly convergent power series (14.15) there whose coefficients are the same functions as those appearing in Theorem 4.2.2.

Theorem 4.3.3. If we rewrite the formal solution (II.3) in the form (III. 3), the coefficients $\left(P_{h}^{3}(x, v), Q_{k}^{(3)}(x, v)\right)(k=0,1, \cdots)$ are funciions holomorphic and bounded in $(x, v)$ for

$$
|x|<a_{0}^{\prime}, \quad|v|<b_{0}^{\prime}
$$

and admitting uniformly convergent expansions of the form (14.16) (whose coefficients are also developable in uniformly 
convergent power series of $v$ ).

In the case when $m=0$, they are holomorphic and bounded in $(x, v)$ for

$$
|x|<a_{0}^{\prime}, \quad|n \arg v+\arg \beta|<\frac{3 \pi}{2}, \quad 0<|v|<b_{0}^{\prime}
$$

and developable there in uniformly convergent power series of the form (14.16) (whose coefficients are functions asymptotically developable in powers of $v$ in the domain $\left(14.12^{\prime}\right)$ ).

\section{Section I. Proof of Theorem 4.2.1.}

\section{Preliminaries.}

To determine the coefficients $\left(P_{k l}(x), Q_{k l}(x)\right),{ }^{5)}$ we shall look for the differential equations which must be satisfied by these functions.

For convenience we rewrite the differential equations (1.1) at this point:

$$
x y^{\prime}=f(x, y, z), \quad x z^{\prime}=g(x, y, z),{ }^{6}
$$

where $f(x, y, z)$ and $g(x, y, z)$ are functions holomorphic and bounded in $(x, y, z)$ for

$$
|x|<a, \quad|y|<b, \quad|z|<b .
$$

Differentiating both sides of (II. 1 ) with respect to $x$, it is easily found that

$$
\begin{aligned}
& f(x, y, z) \sim x U^{\prime}+\sum\left\{x P_{k l}^{\prime}+\left(k \frac{x U^{\prime}}{U}+l \frac{x V^{\prime}}{V}\right) P_{k l}\right\} U^{k} V^{l}, \\
& g(x, y, z) \sim x V^{\prime}+\sum\left\{x Q_{k l}^{\prime}+\left(k \frac{x U^{\prime}}{U}+l \frac{x V^{\prime}}{V}\right) Q_{k l}\right\} U^{k} V^{\imath},
\end{aligned}
$$

where $x U^{\prime}, x V^{\prime}$ must be replaced by the expressions of the right-hand members of the reduced system (2.3) or (2.3') ac-

5) To simplify the description, we write hereafter $P_{k l}(x)$ instead of $P_{k l}^{(1)}(x)$.

6) The symbol' denotes the derivative with the obvious exception $\alpha^{\prime}$ and $\beta^{\prime}$. 
cording as we have Case A or Case B.

Hence we must discuss Case A and Case B separately.

16. Determination of the functions $P_{k l}(x)$ and $Q_{k l}(x)$ for Case $\mathbf{A}$. Inserting (2.3) into (15.3) and (15.4), we obtain (16. 1)

$$
\begin{aligned}
f(x, y, z) & \sim U^{m+1} V^{n}\left(\alpha+\alpha^{\prime} U^{m} V^{n}\right)+\sum\left\{x P_{k l}^{\prime}\right. \\
& \left.+U^{m} V^{n}\left(k \alpha+l \beta+\left(k \alpha^{\prime}+l \beta^{\prime}\right) U^{m} V^{n}\right) P_{k l}\right\} U^{k} V^{l}
\end{aligned}
$$

and

$$
\begin{aligned}
g(x, y, z) & \sim U^{m} V^{n+1}\left(\beta+\beta^{\prime} U^{m} V^{n}\right)+\sum\left\{x Q_{k l}^{\prime}\right. \\
& \left.+U^{m} V^{n}\left(k \alpha+l \beta+\left(k \alpha^{\prime}+l \beta^{\prime}\right) U^{m} V^{n}\right) Q_{k l}\right\} U^{k} V^{\imath} .
\end{aligned}
$$

If we substitute the formal series (II. 1) for $y$ and $z$ appearing in the left-hand members of the relations above, (16.1) and (16.2) can be regarded as formal identities between formal power series with respect to $U$ and $V$ whose coefficients are functions of $x$. Comparing the coefficients of the terms $U^{k} V^{\imath}$ in such formal identities, we can derive the following differential equations :

$$
x P_{00}^{\prime}=f\left(x, P_{00}, Q_{00}\right), \quad x Q_{00}^{\prime}=g\left(x, P_{00}, Q_{00}\right)
$$

and

(16. $4 . k l)$

$$
\left\{\begin{aligned}
x P_{k l}^{\prime}= & \frac{\partial}{\partial y} f\left(x, P_{00}(x), Q_{00}(x)\right) \cdot P_{k l} \\
& +\frac{\partial}{\partial z} f\left(x, P_{00}(x), Q_{00}(x)\right) \cdot Q_{k l}+R_{k l}(x), \\
x Q_{k l}^{\prime}= & \frac{\partial}{\partial y} g\left(x, P_{00}(x), Q_{00}(x)\right) \cdot P_{k l} \\
& +\frac{\partial}{\partial z} g\left(x, P_{00}(x), Q_{00}(x)\right) \cdot Q_{k l}+S_{k l}(x),
\end{aligned}\right.
$$

where

$$
\left\{\begin{aligned}
R_{k l}= & -((k-m) \alpha+(l-n) \beta) P_{k-m l-n}(x) \\
& -\left((k-2 m) \alpha^{\prime}+(l-2 n) \beta^{\prime}\right) P_{k-2 m, l-2 n}(x) \\
& +\mathfrak{P}_{k l}\left(f ; P_{k^{\prime} l^{\prime}}(x), Q_{k^{\prime} l^{\prime}}(x)\right), \\
S_{k l}= & -((k-m) \alpha+(l-n) \beta) Q_{k-m l-n}(x) \\
& -\left((k-2 m) \alpha^{\prime}+(l-2 n) \beta^{\prime}\right) Q_{k-2 m, l-2 n}(x) \\
& +\mathfrak{Q}_{k l}\left(g ; P_{k^{\prime} l^{\prime}}(x), Q_{k^{\prime} l^{\prime}}(x)\right), \text { for }(k, l) \neq(0,0) .
\end{aligned}\right.
$$


Here $\mathfrak{H}_{k l}\left(f ; p_{k^{\prime} \imath}, q_{n^{\prime} l^{\prime}}\right)$ are linear forms of

$$
-\frac{\partial^{k^{\prime}} \neg l^{\prime}}{\partial y^{l^{\prime}} \partial z^{l}} f\left(x, P_{00}(x), Q_{00}(x)\right),\left(k^{\prime}, l^{\prime}\right)<(k, l),,^{7)}
$$

whose coefficients are polynomials of $\left(p_{n^{\prime} l^{\prime}}, q_{k^{\prime} l^{\prime}}\right)$ for $\left(k^{\prime}, l^{\prime}\right)^{7)}$ $(k, l),{ }^{7)}$ and $\Omega_{k l}\left(g ; p_{k^{\prime} l^{\prime}}, q_{h^{\prime} l^{\prime}}\right)$ have the same properties as $\mathfrak{P}_{k l}$.

Moreover, these differential equations admit a formal solution of the form

$$
P_{k l} \sim \sum_{j=0}^{\infty} P_{j k l} x^{j} . \quad Q_{k l} \sim \sum_{j=0}^{\infty} Q_{j k l} x^{j} .
$$

A singularity $x=0$ of the system (16.3) has a character similar to a regular singular point for the linear system. In such a case, we know that the formal solution (16.6.00) has a positive radius of convergence and represents a solution of (16.3) (See, for example, M. Hukuhara [8]). Suppose now that the functions $\left(P_{k l}(x), Q_{k l}(x)\right)$ have been already determined for $(k, l)<(K, L)$ in such a way that they are holomorphic and bounded for $|x|<a_{0}^{\prime}$ and satisfy the differential equations (16.4. kl). Then, since one of $m$ and $n$ is supposed to be positive, the expressions $R_{K L}(x)$ and $S_{K L}(x)$ are known functions defined in $|x|<a_{0}$. Hence, the system (16.4.KL) is a linear system with a regular singularity at $x=0$. Therefore, the formal solution (16.6. KL) is uniformly convergent for $\mid x_{i}^{\prime}<a_{0}^{\prime}$ so that it represents a solution of (16.4.KL). We denote it by $\left(P_{K L}(x), Q_{K L}(x)\right)$.

Thus the functions $\left(P_{k l}(x), Q_{k l}(x)\right)$ are all holomorphic and bounded in $|x|<a_{0}^{\prime}$.

17. Determination of the functions $P_{k l}(x)$ and $Q_{x l}(x)$ for Case $\mathbb{B}$.

In this case, since the reduced system is given by $\left(2.3^{\prime}\right)$, we can easily verify that the differential equations which the functions $P_{k l}(x)$ and $Q_{k l}(x)$ must satisfy are given by the same

7) If $k^{\prime} \leqq k$ and $l^{\prime}<l$ or $k^{\prime}<k$ and $l^{\prime} \leqq l$, we say that the arrangement $\left(k^{\prime} . l^{\prime}\right)$ precedes the arrangement $(k, l)$ and write $\left(k^{\prime}, l^{\prime}\right)<(k, l)$. 
form as (16.3) and (16.4.kl) if we replace the functions $R_{k l}(x)$ and $S_{k l}(x)$ by :

$$
\begin{aligned}
R_{k l}(x)= & -((k-m) \gamma \mu+(l-n) \gamma \nu) P_{k-m l-n}(x) \\
& -\sum_{-{ }_{\nu} \leq n \leq \leq_{\mu}^{n}}\left((k-2 m-\nu h) \gamma_{h} \mu+(l-2 n+\mu h) \gamma_{h^{\nu}}\right) \\
& \times P_{k-2 m-\nu h, l-2 n+\mu h}(x)+\psi_{k l}^{2}\left(f ; P_{k^{\prime} l^{\prime}}(x), Q_{k^{\prime} l}(x)\right), \\
S_{k l}(x)= & -((k-m) \gamma \mu+(l-n) \gamma \nu) Q_{k-m l-n}(x) \\
& -\sum_{-\frac{m}{\nu} \leq n \leq \frac{n}{\mu}}\left((k-2 m-\nu h) \gamma_{h} \mu+(l-2 n+\mu h) \gamma_{h^{\nu}} \nu\right) \\
& \times Q_{k-2 m-\nu h, l-2 n+\mu h}(x)+\mathfrak{Q}_{k l}\left(g ; P_{k^{\prime} l^{\prime}}(x), Q_{k^{\prime} l^{\prime}}(x)\right) .
\end{aligned}
$$

Here we must notice that there exists a formal solution of the form $(16.6 . k l)$ and one of the quantities $m+\nu h(\geqq 0)$ and $n-\mu h(\geqq 0)$ is positive. Hence, we can conclude that this formal solution is uniformly convergent for $|x|<a_{0}^{\prime}$ and represents a solution of the corresponding differential equations.

Thus we have proved Theorem 4.2.1.

Section II. Proof of Theorems 4.2.2 and 4.2.3.

\section{Preliminaries.}

Since the discussion of Theorem 4.2 .3 can be carried out in quite a similar way, we shall prove Theorem 4.2.2 only.

We notice that the system (1.1) can be written as

$$
x y^{\prime}=f_{0}(y, z)+f_{1}(x, y, z), \quad x z^{\prime}=g_{0}(y, z)+g_{1}(x, y, z)
$$

with

$$
\begin{cases}f_{0}(y, z)=y^{n+1} z^{n} f^{*}(y, z), \quad g_{0}(y, z)=y^{n} z^{n+1} g^{*}(y, z) \\ f^{*}(0,0)=\alpha, & g^{*}(0,0)=\beta \\ f_{1}(0, y, z) \equiv 0, & g_{1}(0, y, z) \equiv 0\end{cases}
$$

where $f^{*}(y, z), g^{*}(y, z), f_{1}(x, y, z)$ and $g_{1}(x, y, z)$ are functions holomorphic and bounded in $(x, y, z)$ for

$$
|x|<a, \quad|y|<b, \quad|z|<b .
$$


Now we determine differential equations which must be satisfied by the functions $\left(P_{l}(u), Q_{l}(u)\right)$ and $\left(P_{j l}(u), Q_{j l}(u)\right)$ $(j \geqq 1)$.

Termwise differentiation of the formal series (II.2) will yield :

$$
\begin{aligned}
x y^{\prime} \sim x U^{\prime} & \left(1+\sum_{l=0}^{\infty} P_{l}(U) V^{\imath}\right)+U \sum_{l=0}^{\infty}\left(\frac{d}{d U} P_{l}(U) \cdot x U^{\prime}\right. \\
& \left.+P_{l}(U) \cdot l \frac{x V^{\prime}}{V}\right) V^{\imath}+\sum_{l \geq 1 . l \geq 0}\left\{\frac{d}{d U^{\prime}} P_{l l}(U) \cdot x U^{\prime}\right. \\
& \left.+\left(j+l \frac{x V^{\prime}}{V}\right) P_{\jmath l}(U)\right\} x^{j} V^{\imath},
\end{aligned}
$$

$$
\begin{aligned}
x z^{\prime} \sim x V^{\prime}(1 & \left.+\sum_{l=0}^{\infty} Q_{l}(U) V^{l}\right)+V \sum_{l=0}^{\infty}\left(\frac{d}{d U^{\prime}} Q_{l}(U) \cdot x U^{\prime}\right. \\
& \left.\left.+Q_{l}(U) \cdot l \frac{x V^{\prime}}{V}\right) V^{l}+\sum_{j \geq 1, l \geq 0}\right\} \frac{d}{d U^{\prime}} Q_{\jmath}(U) \cdot x U^{\prime} \\
& \left.+\left(j+l \frac{x V^{\prime}}{V}\right) Q_{j l}(U)\right\} x^{j} V^{\imath},
\end{aligned}
$$

where $\left(x U^{\prime}, x V^{\prime}\right)$ must be replaced by the expressions of the right-hand members of the reduced system (2.3) or $\left(2.3^{\prime}\right)$ according as we have Case A or Case B.

19. Differential equations which determine $\left(P_{l}(u), Q_{l}(u)\right)$ and $\left(P_{j l}(u), Q_{j l}(u)\right)(j \geqq 1)$ for Case A.

From (18.1) we see that (18.3) and (18.4) are reduced to

$$
\begin{aligned}
y^{m+1} z^{n} f^{*}(y, z) & +f_{1}(x, y, z) \sim U^{m+1} V^{n}\left(\alpha+\alpha^{\prime} U^{m} V^{n}\right) \\
& +U^{m+1} V^{n} \sum_{l=0}^{\infty}\left\{U\left(\alpha+\alpha^{\prime} U^{m} V^{n}\right) \frac{d}{d U^{\prime}} P_{l}\right. \\
& \left.+\left(\alpha+l \beta+\left(\alpha^{\prime}+l \beta^{\prime}\right) U^{m} V^{n}\right) P_{l}\right\} V^{l} \\
& +\sum_{j \geq 1, l \geq 0}\left\{U^{m+1} V^{n}\left(\alpha+\alpha^{\prime} U^{m} V^{n}\right) \frac{d}{d U^{\prime}} P_{j l}\right. \\
& \left.+\left(j+l U^{m} V^{n}\left(\beta+\beta^{\prime} U^{m} V^{n}\right)\right) P_{j l}\right\} x^{j} V^{l}
\end{aligned}
$$

and

$$
\begin{aligned}
y^{m} z^{n+1} g^{*}(y, z) & +g_{1}(x, y, z) \sim U^{m} V^{n+1}\left(\beta+\beta^{\prime} U^{m} V^{n}\right) \\
& +U^{m} V^{n+1} \sum_{l=0}^{\infty}\left\{U\left(\alpha+\alpha^{\prime} U^{m} V^{n}\right) \frac{d}{d U^{Q_{l}}}\right.
\end{aligned}
$$




$$
\begin{aligned}
& \left.+(l+1)\left(\beta+\beta^{\prime} U^{m} V^{n}\right) Q_{l}\right\} V^{l} \\
& +\sum_{\jmath \geqq 1, l \geq 0}\left\{U^{m+1} V^{n}\left(\alpha+\alpha^{\prime} U^{m} V^{n}\right) \frac{d}{d U^{\prime}} Q_{j l}\right. \\
& \left.+\left(j+l U^{m} V^{n}\left(\beta+\beta^{\prime} U^{m} V^{n}\right)\right) Q_{j l}\right\} x^{j} V^{\imath} .
\end{aligned}
$$

If we substitute the formal solution (II.2) for $(y, z)$ appearing in the left-hand members of the equations above, then we obtain formal identities between the formal power series of $x$ and $V$ whose coefficients are functions of $U$.

$1^{\circ}$. The differential equations satisfied by $P_{l}(u)$ and $Q_{l}(u)$ $(l=0,1, \cdots)$.

Equating the coefficients of the terms $U^{m+1} V^{n+l}$ and $U^{m} V^{n+1+l}$ in the first and the second of these formal identities and replacing $U$ by $u$, we are able to find the differential equations which must be satisfied by $P_{l}(u)$ and $Q_{l}(u)$. After a simple calculation we see that these differential equations are given as follows :

Case when $n>0$.

$$
\left\{\begin{array}{l}
\alpha u P_{0}^{\prime}=\left(1+P_{0}\right)^{m+1}\left(1+Q_{0}\right)^{n} f^{*}\left(u+u P_{0}, 0\right)-\alpha-\alpha P_{0}, \\
\alpha u Q_{0}^{\prime}=\left(1+P_{0}\right)^{m}\left(1+Q_{0}\right)^{n+1} g^{*}\left(u+u P_{0}, 0\right)-\beta-\beta Q_{0},
\end{array}\right.
$$

and

$$
\left\{\begin{array}{l}
\alpha u P_{l}^{\prime}=\left(C_{11}(u)-\alpha-l \beta\right) P_{l}+C_{12}(u) Q_{l}+R_{l}(u), \\
\alpha u Q_{l}^{\prime}=C_{21}(u) P_{l}+\left(C_{22}(u)-(1+l) \beta\right) Q_{l}+S_{l}(u),
\end{array}\right.
$$

where

$$
\begin{aligned}
& C_{11}(u)=\left[\frac{\partial}{\partial \bar{y}}\left(\bar{y}^{m+1} \bar{z}^{n} f^{*}(u \bar{y}, 0)\right)\right]_{\bar{y}=1+P 0(u), \bar{z}=1+Q_{0}(u)}, \\
& C_{12}(u)=\left[\frac{\partial}{\partial \bar{z}}\left(\bar{y}^{m+1} \bar{z}^{n} f^{*}(u \bar{y}, 0)\right)\right]_{\bar{y}=1+P_{0}(u), \bar{z}=1+Q_{0}(u)}, \\
& C_{21}(u)=\left[\frac{\partial}{\partial \bar{y}}\left(\bar{y}^{m} \bar{z}^{n+1} g^{*}(u \bar{y}, 0)\right)\right]_{\bar{y}=1+P_{0}(u), \bar{z}=1+Z_{0}(u)}, \\
& C_{22}(u)=\left[\frac{\partial}{\partial \bar{z}}\left(\bar{y}^{m} \bar{z}^{n+1} g^{*}(u \bar{y}, 0)\right)\right]_{\bar{y}=1+P_{0}(u), \bar{z}=1+Q_{0}(u)}, \\
& \left(R_{l}(u)=-\alpha^{\prime} u^{m} P_{l-n}^{\prime}(u)-\left(\alpha^{\prime}+(l-n) \beta^{\prime}\right) u^{m} P_{l-n}(u)\right.
\end{aligned}
$$




$$
\left\{\begin{array}{c}
-\alpha^{\prime} u^{m} \cdot \delta_{n, l}+\mathfrak{P}_{l}\left(f_{0} ; P_{l^{\prime}}(u), Q_{l^{\prime}}(u)\right), \\
S_{l}(u)=-\alpha^{\prime} u^{m} Q_{l-n}^{\prime}(u)-(l-n+1) \beta^{\prime} u^{m} Q_{l-n}(u) \\
-\beta^{\prime} u^{m} \cdot \delta_{n, l}+\mathfrak{\Omega}_{l}\left(g_{0} ; P_{l}(u), Q_{l^{\prime}}(u)\right) .
\end{array}\right.
$$

Here $\delta_{n, l}=0(l \neq n),=1(l=n) ; \mathfrak{F}_{l}\left(f_{0} ; p_{l^{\prime}}, q_{l}\right)$ is a linear form of the functions

$$
\left[\frac{\partial^{k+l^{\prime}}}{\partial \bar{y}^{k} \partial \bar{z}^{l^{\prime}}}\left(\bar{y}^{m+1} f_{i}^{*}(u \bar{y}) \bar{z}^{n+t}\right)\right]_{\bar{y}=1+P_{0}(u), \bar{z}=1+Q_{0}(u)},
$$

$\left(i+k^{\prime}+l^{\prime} \leqq l ;\left(i, k^{\prime}, l^{\prime}\right) \neq(0,1,0), \neq(0,0,1)\right)$, whose coefficients are polynomials of $p_{l^{\prime}}$ and $q_{l^{\prime}}\left(1 \leqq l^{\prime}<l\right)$, where the $f_{i}^{*}(y)$ are the coefficients of $z^{i}$ in the uniformly convergent expansion of the function $f^{*}(y, z)$ in power series of $z$. The expression $\mathfrak{\Omega}_{l}\left(g_{0}\right.$; $\left.p_{l^{\prime}}, q_{l}\right)$ has the same properties as $\mathfrak{F}_{l}\left(f_{0} ; p_{l}, q_{l}\right)$ (where $m+1$ and $n$ must be replaced by $m$ and $n+1$ respectively).

Case when $n=0$.

In this case, as can be easily seen from the above discussion, the differential equations (19.3) and (19.4.l) must be replaced by

$$
\begin{gathered}
\left\{\begin{array}{c}
u\left(\alpha+\alpha^{\prime} u^{m}\right) P_{0}^{\prime}=\left(1+P_{0}\right)^{m+1}\left(1+Q_{0}\right)^{n} f^{*}\left(u+u P_{0}, 0\right) \\
-\left(\alpha+\alpha^{\prime} u^{m}\right)-\left(\alpha+\alpha^{\prime} u^{m}\right) P_{0}, \\
u\left(\alpha+\alpha^{\prime} u^{m}\right) Q_{0}^{\prime}=\left(1+P_{0}\right)^{m}\left(1+Q_{0}{ }^{n+1}\right) g^{*}\left(u+u P_{0}, 0\right) \\
-\left(\beta+\beta^{\prime} u^{m}\right)-\left(\beta+\beta^{\prime} u^{m}\right) Q_{0},
\end{array}\right. \\
\left\{\begin{array}{c}
u\left(\alpha+\alpha^{\prime} u^{m}\right) P_{l}^{\prime}=\left(C_{11}(u)-\alpha-l \beta-\left(\alpha^{\prime}+l \beta^{\prime}\right) u^{m}\right) \cdot P_{l} \\
+C_{12}(u) \cdot Q_{l}+\mathfrak{P}_{l}, \\
u\left(\alpha+\alpha^{\prime} u^{m}\right) Q_{l}=C_{21}(u) \cdot P_{l}+\left(C_{22}(u)-\beta-l \beta\right. \\
\left.-\left(\beta^{\prime}+l \beta^{\prime}\right) u^{m}\right) \cdot Q_{l}+\mathfrak{Q}_{l},
\end{array}\right.
\end{gathered}
$$

where $\mathfrak{F}_{\imath}$ and $\Omega_{l}$ are the same functions as before.

$2^{\circ} \quad$ The differential equations satisfied by $P_{j l}(u)$ and $Q_{j l}(u)$.

Next, comparing the coefficients of the terms $x^{j} V^{l}$ in the formal identities mentioned above, we can find the differential equations which the functions $P_{j l}(u)$ and $Q_{j l}(u)$ must satisfy. A simple calculation shows us that these equations are reduced 
to algebraic equations written as follows:

Case when $n>0$.

(19. 8. $j l)$

$$
\left\{\begin{array}{l}
\left(-j+c_{11}(u)\right) P_{j l}+c_{12}(u) Q_{j l}=R_{j l}(u), \\
c_{21}(u) P_{j l}+\left(-j+c_{22}(u)\right) Q_{j l}=S_{j l}(u),
\end{array}\right.
$$

where

$$
\begin{aligned}
& c_{11}(u)=\frac{\partial}{\partial y} f_{0}\left(u+u P_{0}(u), 0\right), \\
& c_{12}(u)=\frac{\partial}{\partial z} f_{0}\left(u+u P_{0}(u), 0\right) \text {, } \\
& c_{21}(u)=\frac{\partial}{\partial y} g_{0}\left(u+u P_{0}(u), 0\right), \\
& c_{22}(u)=\frac{\partial}{\partial z} g_{0}\left(u+u P_{0}(u), 0\right), \\
& \left\{\begin{aligned}
R_{j l}(u)= & \alpha u^{m+1} P_{\jmath l-n}^{\prime}(u)+\alpha^{\prime} u^{2 m+1} P_{\jmath l-2 n}(u) \\
& +(l-n) \beta u^{m} P_{j l-n}(u)+(l-2 n) \beta^{\prime} u^{2 n n} P_{j l-2 n}(u) \\
& +\mathfrak{S}_{j l}\left(f ; u P_{l}(u), Q_{l}(u), P_{j l}(u), Q_{j l}(u)\right), \\
S_{j l}(u)= & \alpha u^{m+1} Q_{j l-n}^{\prime}(u)+\alpha^{\prime} u^{2 n+1} Q_{\jmath l-2 n}^{\prime}(u) \\
& +(l-n) \beta u^{m} Q_{j l-n}(u)+(l-2 n) \beta^{\prime} u^{2 m} Q_{j l-2 n}(u) \\
& +\mathfrak{\Omega}_{j l}\left(g ; u P_{l}(u), Q_{l}(u), P_{j l}(u), Q_{j l}(u)\right) .
\end{aligned}\right.
\end{aligned}
$$

Here $\mathfrak{F}_{j l}^{\prime}\left(f ; u p_{\imath}, q_{l^{\prime}}, p_{j^{\prime} \imath}, q_{j^{\prime} \imath^{\prime}}\right)$ is a linear form of

$$
\left\{\begin{array}{l}
\frac{\partial^{j+k+l}}{\partial x^{j} \partial y^{k} \partial z^{l^{\prime}}} f\left(0, u+u P_{0}(u), 0\right), \\
j^{\prime}+k^{\prime}+l^{\prime} \leqq j+l, \\
\left(j^{\prime}, k^{\prime}, l^{\prime}\right) \neq(0,1,0), \neq(0,0,1), \\
\left(j^{\prime}, l^{\prime}\right)<(j, l),
\end{array}\right.
$$

whose coefficients are polynomials of $u p_{l}\left(1 \leqq l^{\prime} \leqq l\right), q_{l}\left(0 \leqq l^{\prime} \leqq l-1\right)$, $p_{j l^{\prime}}$ and $q_{j l^{\prime}}\left(\left(j^{\prime}, l^{\prime}\right)<(j, l)\right)$ and the expression $\Omega_{j l}\left(g ; u p_{l^{\prime}}, q_{l}\right.$, $p_{j l}, q_{j l}$ ) has the same properties as $\mathcal{F}_{j l}$ (Of course, $f=f_{0}+f_{1}$, $\left.g=g_{0}+g_{1}\right)$.

Case when $n=0$.

In this case, the functions $P_{j l}$ and $Q_{j l}$ to be determined appear in the expressions of $R_{j l}(u)$ and $S_{j l}(u)$. Hence, (19.8.jl) 
must be replaced by the following differential equations:

(19. 10. $j l)\left\{\begin{array}{r}u^{m+1}\left(\alpha+\alpha^{\prime} u^{m}\right) P_{j l}^{\prime}=\left(-j+c_{11}(u)-l \beta u^{m}-l \beta^{\prime} u^{2 m}\right) \cdot P_{j l} \\ +c_{12}(u) \cdot Q_{j l}-\mathfrak{P}_{j l}, \\ u^{m+1}\left(\alpha+\alpha^{\prime} u^{m}\right) Q_{j l}^{\prime}=c_{21}(u) \cdot P_{j l} \\ +\left(-j+c_{22}(u)-l \beta u^{m}-l \beta^{\prime} u^{2 m}\right) \cdot Q_{j l}-\Omega_{j l} .\end{array}\right.$

20. Determination of the functions $\left(P_{l}(u), Q_{l}(u)\right)$ and $\left(P_{j l}(u)\right.$, $\left.Q_{j l}(u)\right)$ for $\mathbb{C a s e} \mathbb{A}$ and $n>0$.

$1^{\circ}$. The functions $P_{l}(u)$ and $Q_{l}(u)$.

We observe that the differential equations (19.3) and (19.4.l) admit a formal solution of the form

$$
P_{0} \sim \sum_{k=1}^{\infty} P_{0 k 0} u^{k-1}, Q_{0} \sim \sum_{h=0}^{\infty} Q_{0 k 1} u^{k}
$$

and

$$
P_{l} \sim \sum_{k=1}^{\infty} P_{0 k l} u^{k-1}, Q_{l} \sim \sum_{h=0}^{\infty} Q_{0 k l+1} u^{k}
$$

respectively.

Since the differential equations (19.3) have a singularity similar to a regular singularity at $u=0$, it is known that the formal solution of the form (20.1) is uniformly convergent for $|u|<b_{0}$ and represents a holomorphic solution. We write such a solution by $P_{0}(u)$ and $Q_{0}(u)$.

Suppose now that the functions $P_{l}(u)$ and $Q_{l}(u)$ have been already determined as solutions of the differential equations (19.4. $l$ ) for $l<L$ in such a way that they are holomorphic and bounded for $|u|<b_{0}$ and, moreover, admit uniformly convergent expansions of the form (20.2.l). Then the expressions $R_{L}(u)$ and $S_{L}(u)$ are known functions. Hence the differential equations (19.4. $L$ ) become a linear system with a regular singular point at $u=0$, and they admit a formal solution of the form (20.2. L). Consequently, such a formal solution is uniformly convergent for $|u|<b_{0}$ and represents a holomorphic and bounded solution 
of the system (19.4.L). We denote it by $\left(P_{L}(u), Q_{L}(u)\right)$.

$2^{\circ}$. The functions $P_{j l}(u)$ and $Q_{j l}(u)$.

We notice that the equations (19.8. $j l$ ) are algebraic equations and they admit a formal solution of the form

$$
P_{j l} \sim \sum_{k=0}^{\infty} P_{j k l} u^{k}, \quad Q_{j l} \sim \sum_{h=0}^{\infty} Q_{j k l} u^{k} .
$$

Moreover, since $n>0$, we have evidently

$$
c_{11}(u)=c_{12}(u)=c_{21}(u)=c_{22}(u) \equiv 0 .
$$

Consequently, the matrix formed by the coefficients of $P_{j l}$ and $Q_{j l}$ in $(19.8 . j l)$ is written as $-j \cdot \mathbb{1}_{2}$ and therefore is non-singular, where $\mathbb{1}_{2}$ is the 2 -by-2 unit-matrix.

Suppose now that the functions $P_{j l}(u)$ and $Q_{j l}(u)$ have been already determined for $(j, l)<(j, L)$ in such a way that they are holomorphic and bounded for $|u|<b_{0}$ and satisfy the algebraic equations (19.8. $j l$ ). Then it is clear that the convergent expansions of such functions coincide with (20.3. $j l$ ), and the expressions $R_{J L}(u)$ and $S_{J L}(u)$ are known functions. From this we see that the functions $P_{J L}(u)$ and $Q_{J L}(u)$ can be uniquely determined by the algebraic equations (19.8. JL). Clearly, these functions are holomorphic and bounded for $|u|<b_{0}$ and their convergent expansions coincide with the formal series (20.3. JL).

21. Determination of $\left(P_{l}(u), Q_{l}(u)\right)$ and $\left(P_{j l}(u), Q_{j l}(u)\right)$ for Case A and $n=0$.

In this case, the differential equations which determine the functions $P_{0}(u)$ and $Q_{0}(u)$ are given by (19.6) and those which determine the functions $P_{l}(u)$ and $Q_{l}(u)(l \geqq 1)$ are given by (19.7.l). Moreover, these differential equations admit a formal solution of the form (20.1) and (20.2.l) respectively. Hence, by the same method as before, we can aetermine the functions $P_{l}(u)$ and $Q_{l}(u)(l \geqq 0)$ with the desired properties.

But, the determination of the functions $P_{j l}(u)$ and $Q_{j l}(u)$ 
differs from that for the case when $n>0$.

Indeed, the differential equations which determine these functions are given by $(19.10 . j l)$ and its singularity $u=0$ is similar to an irregular singularity for the linear system. Consequently, though the system (19.10. $j l$ ) admits a formal solution of the form (20.3. $j l$ ), it is generally divergent. On the other hand, it is easy to see that the matrix formed by the coefficients of the linear terms of $P_{j l}$ and $Q_{j l}$ is reduced to a diagonal matrix $-j \cdot \mathbb{1}_{2}$ at $u=0$, where $\mathbf{1}_{2}$ denotes the 2 -dimensional unitmatrix. And, it is to be noticed that if $u$ is in a domain

$$
|m \arg u+\arg \alpha|<\frac{3 \pi}{2}, \quad 0<|u|<b_{0}^{\prime},
$$

we have the inequality

$$
\left|\arg \left(-\int_{\infty}^{u} \frac{j}{\left(\alpha+\alpha^{\prime} u^{m}\right) u^{m+1}} d u\right)\right|<\frac{3 \pi}{2} .
$$

Hence, by virtue of a theorem due to $M$. Iwano [3], which is essentially due to M. Hukuhara [9], we are led to the following conclusion :

Suppose that the expressions $\mathfrak{X}_{J L}$ and $\Sigma_{J L}$ are functions holomorphic and bounded for (14.11') and, moreover, asymptotically developable in powers of $u$. Then, the differential equations (19.10. JL) admit a solution $\left(P_{J L}(u), Q_{J L}(u)\right)$, where $P_{J L}(u)$ and $Q_{J L}(u)$ are functions holomorphic and bounded for (14.11') and asymptotically developable in the form (20.3. JL) as u tends to 0 in the domain (14.11').

Thus, Theorem 4.2.2 has been completely established for Case A.

22. Determination of the functions $\left(P_{l}(u), Q_{l}(u)\right)$ and $\left(P_{j l}(u)\right.$, $\left.Q_{j l}(u)\right)$ for Case $\mathrm{B}$.

In this case, concerning the quantities $\gamma_{h}$ appearing in the reduced system $\left(2.3^{\prime}\right)$, we notice that the condition $\gamma_{h} \neq 0$ implies that $m+\nu h$ and $n-\mu h$ are both nonnegative integers not simul- 
taneously zero. And, the quantity $n-\mu h$ may vanish, if at all, for only one value of $h$, say $h_{0}$ and, consequently, the quantity $m+\nu h_{0}$ is positive.

By applying the reasoning similar to that used to derive the differential equations (19.3) and (19.4.l), we can prove the following proposition :

The differential equations which determine the functions $P_{l}(u)$ and $Q_{l}(u)(l=0,1, \cdots)$ are given by

$$
\begin{gathered}
\left\{\begin{array}{r}
\mu \gamma(u) u P_{0}^{\prime}=\left(1+P_{0}\right)^{m+1}\left(1+Q_{0}\right)^{n} f^{*}\left(u+u P_{0}, 0\right) \\
-\mu \gamma(u)-\mu \gamma(u) P_{0}, \\
\mu \gamma(u) u Q_{0}^{\prime}=\left(1+P_{0}\right)^{m}\left(1+Q_{0}\right)^{n+1} g^{*}\left(u+u P_{0}, 0\right) \\
-\nu \gamma(u)-\nu \gamma(u) Q_{0},
\end{array}\right. \\
\left\{\begin{array}{r}
\mu \gamma(u) u P_{l}^{\prime}=\left(C_{11}(u)-\left(\mu+l_{\nu}\right) \gamma(u)\right) \cdot P_{l} \\
+C_{12}(u) \cdot Q_{l}+R_{l}(u), \\
\mu \gamma(u) u Q_{l}^{\prime}=C_{21}(u) \cdot P_{l}+\left(C_{22}(u)\right. \\
\left.-\left(l_{\nu}+\nu\right) \gamma(u)\right) \cdot Q_{l}+S_{l}(u),
\end{array}\right.
\end{gathered}
$$

where

$$
\begin{aligned}
\gamma(u)= & \gamma+\gamma_{h_{0}} u^{m+\nu h_{0}} \quad\left(n-\nu h_{0}=0\right), \\
R_{l}(u)= & -\sum_{n \neq n_{0}} \gamma_{h} u^{m+\nu h}\left\{\mu u P_{l-n+\mu h}^{\prime}(u)\right. \\
& \left.+(\mu+\nu(l-n+\mu h)) P_{l-n+\mu h}(u)\right\}-\mu \gamma_{h} u^{m+\mu h^{\prime}}+\Re_{l} . \\
S_{l}(u)= & -\sum_{n \neq n_{0}} \gamma_{h} u^{m+\nu h}\left\{\mu u Q_{l-n+\mu h}^{\prime}(u)\right. \\
& \left.+\nu(l+1-\mu n+h) Q_{l-n+\mu h}(u)\right\}-\nu \gamma_{n} u^{m+\mu h^{\prime}}+\Omega_{l} .
\end{aligned}
$$

Here $h^{\prime}$ is an integer, if it exists, such that $n-\nu h^{\prime}=l$, and $\mathfrak{F}_{z}$ and $\mathfrak{D}_{2}$ are the same functions as those that appeared in the formulae (19.5). Moreover. these systems admit a formal solution of the form (20.1) and (20.2.l) respectively.

Hence these systems have the same characters as the systems (19.3) and (19.4.l) respectively, which have been already studied in 20.

Furthermore we can verify that the differential equations which are to be satisfied by the functions $P_{j l}(u)$ and $Q_{j l}(u)$ are 
written in the same form as (19.8.jl), where the expressions $R_{j z}(u)$ and $S_{j l}(u)$ must be replaced by

$$
\begin{aligned}
R_{j l}(u)= & \mu u^{m+1} \gamma(u) P_{\jmath l-n}^{\prime}(u)+(l-n) \nu u^{m} \gamma(u) P_{j l-n}(u) \\
& +\sum_{h \neq n_{0}} \gamma_{h} u^{2 m+\nu h}\left\{\mu u P_{j, l-2 n+\mu h}^{\prime}(u)\right. \\
& \left.+\nu(l-2 n+\mu h) P_{j, l-2 n+\mu h}(u)\right\}+\Re_{j l}, \\
S_{j l}(u)= & \mu u^{m+1} \gamma(u) Q_{j l-n}^{\prime}(u)+\nu(l-n) u^{m} \gamma(u) Q_{j l-n}(u) \\
& +\sum_{h \neq n_{0}} \gamma_{h} u^{2 m+\nu h}\left\{\mu u Q_{j, l-2 n+\mu h}(u)\right. \\
& \left.+\nu(l-2 n+\mu h) Q_{j, l-2 n+\mu h}(u)\right\}+\Omega_{j l} .
\end{aligned}
$$

Here $\mathfrak{P}_{j z}$ and $\Omega_{j l}$ are the same functions as those that appeared in (19.9). Moreover, this system admits a formal solution of the form (20.3. $j l)$.

Hence, it follows immediately that, in the case when $n>0$, those differential equations are reduced to algebraic equations with respect to $P_{j l}(u)$ and $Q_{j l}(u)$. But, in the case when $n=0$, those systems are reduced to linear differential equations of the following form with an irregular singular point at $u=0$ :

$$
\left\{\begin{array}{l}
\mu \gamma(u) u^{m+1} P_{\jmath l}^{\prime}=\left(-j+c_{11}(u)-l \nu u^{m} \gamma(u)\right) \cdot P_{j l}+c_{12}(u) \cdot Q_{j l}+R_{j l}, \\
\mu \gamma(u) u^{m+1} Q_{\jmath l}^{\prime}=c_{21}(u) \cdot P_{j l}+\left(-j+c_{22}(u)-l \nu u^{m} \gamma(u)\right) \cdot Q_{j l}+S_{j l},
\end{array}\right.
$$

where

$$
\left\{\begin{array}{l}
R_{j l}=-\sum_{h \neq h_{0}} \gamma_{h} \cdot u^{2 m+\nu h}\left\{\mu u P_{\jmath l+\mu h}^{\prime}(u)+\nu(l+\mu h) P_{\jmath l+\mu h}(u)\right\}-\mathfrak{P}_{j l}, \\
S_{j l}=-\sum_{h \neq h_{0}} \gamma_{h} \cdot u^{2 m+\nu h}\left\{\mu u Q_{\jmath l+\mu h}^{\prime}(u)+\nu(l+\mu h) Q_{j l+\mu h}(u)\right\}-\Omega_{j l},
\end{array}\right.
$$

and, moreover, this system admits a formal solution of the form (20. 3. $j l$ ).

Therefore, applying the same reasoning as in Case $\mathrm{A}$, we can give the analytical meaning stated in the Theorem to such a formal solution.

Thus Theorem 4.2.2 has been proved for Case B also. 
Section III. Proof of Theorem 4.3.1.

\section{Preliminaries.}

The system (1.1) can be written as

$$
\begin{aligned}
& x y^{\prime}=\sum_{j=0}^{\infty} f_{j}(y, z) x^{j}, x z^{\prime}=\sum_{j=0}^{\infty} g_{j}(y, z) x^{j}, \\
& f_{0}(y, z)=y^{m+1} z^{n} f^{*}(y, z), f^{*}(0,0)=\alpha \\
& g_{0}(y, z)=y^{m} z^{n+1} g^{*}(y, z), g^{*}(0,0)=\beta
\end{aligned}
$$

Here the power series in the right-hand members are uniformly convergent in a neighborhood of $(0,0,0)$ and, moreover, $f^{*}(y, z), g^{*}(y, z), f_{j}(y, z)$ and $g_{j}(y, z)$ are functions holomorphic and bounded in $(y, z)$ at $(0,0)$.

Let us seek differential equations which must be satisfied by $P_{j}(u, v)$ and $Q_{j}(u, v)(j=0,1, \cdots)$.

By differentiating both sides of the formal solution (III.1) term by term, we obtain formally

$$
\begin{aligned}
x y^{\prime} \sim x U^{\prime}(1 & \left.+P_{0}(U, V)\right)+U \cdot x \frac{d P_{0}(U, V)}{d x} \\
& +\sum_{j=1}^{\infty}\left\{j P_{j}(U, V)+x \frac{d P_{j}(U, V)}{d x}\right\} x^{j} \\
x z^{\prime} \sim x V^{\prime}(1+ & \left.Q_{0}(U, V)\right)+V \cdot x \frac{d Q_{0}(U, V)}{d x} \\
& +\sum_{j=1}^{\infty}\left\{j Q_{j}(U, V)+x \frac{d Q_{j}(U, V)}{d x}\right\} x^{j}
\end{aligned}
$$

with identities

$$
\left\{\begin{array}{l}
x \frac{d P_{j}}{d x}=\frac{\partial P_{j}(U, V)}{\partial U} \cdot x U^{\prime}+\frac{\partial P_{j}(U, V)}{\partial V} \cdot x V^{\prime} \\
x \frac{d Q_{j}}{d x}=\frac{\partial Q_{j}(U, V)}{\partial U} \cdot x U^{\prime}+\frac{\partial Q_{j}(U, V)}{\partial V} \cdot x V^{\prime}
\end{array}\right.
$$

where $x U^{\prime}$ and $x V^{\prime}$ must be replaced by the expressions in the right-hand members of (2.3) or (2.3') according as we have Case A or Case B. 
By virtue of (23.4), the expressions $x \cdot d P_{j}(U, V) / d x$ and $x \cdot d Q_{j}(U, V) / d x$ are regarded as functions of $U$ and $V$ only.

Substituting the formal solution (III. 1) for $y$ and $z$ appearing in the expressions of the right-hand members of $\left(x y^{\prime}, x z^{\prime}\right)$, the equations (23.2) and (23.3) will be reduced to formal identities between formal power series of $x$ whose coefficients are formal power series of $U$ and $V$. Hence, comparing the coefficients of the terms $x^{j}(j=0,1, \cdots)$ of these formal identities, we can find differential equations which must be satisfied by the functions $P_{j}(U, V)$ and $Q_{j}(U, V)(j=0,1, \cdots)$.

Since the discussion for Case B can be carried out in quite a similar way, we discuss the Case A only.

24. Differential equations satisfied by $P_{j}$ and $Q_{j}$.

A simple calculation shows us that these differential equations are given by

$$
\left\{\begin{array}{c}
x P_{0}^{\prime}=U^{m} V^{n}\left(1+P_{0}\right)^{m+1}\left(1+Q_{0}\right)^{n} f^{*}\left(U\left(1+P_{0}\right), V\left(1+Q_{0}\right)\right) \\
-U^{m} V^{n}\left(\alpha+\alpha^{\prime} U^{m} V^{n}\right)\left(1+P_{0}\right), \\
x Q_{0}^{\prime}=U^{m} V^{n}\left(1+P_{0}\right)^{m}\left(1+Q_{0}\right)^{n+1} g^{*}\left(U\left(1+P_{0}\right), V\left(1+Q_{0}\right)\right) \\
-U^{m} V^{n}\left(\beta+\beta^{\prime} U^{m} V^{n}\right)\left(1+Q_{0}\right),
\end{array}\right.
$$

$$
\text { 2.j) }\left\{\begin{array}{l}
x P_{j}^{\prime}=\left(-j+C_{11}(U, V)\right) \cdot P_{j}+C_{12}(U, V) \cdot Q_{j}+R_{j}(U, V), \\
x Q_{j}^{\prime}=C_{21}(U, V) \cdot P_{j}+\left(-j+C_{22}(U, V)\right) \cdot Q_{j}+S_{j}(U, V) .
\end{array}\right.
$$

Here

$$
\left\{\begin{array}{l}
C_{11}(u, v) \equiv \frac{\partial}{\partial y} f_{0}\left(u+u P_{0}(u, v), v+v Q_{0}(u, v)\right) \\
C_{12}(u, v) \equiv \frac{\partial}{\partial z} f_{0}\left(u+u P_{0}(u, v), v+v Q_{0}(u, v)\right) \\
C_{21}(u, v) \equiv \frac{\partial}{\partial y} g_{0}\left(u+u P_{0}(u, v), v+v Q_{0}(u, v)\right) \\
C_{22}(u, v) \equiv \frac{\partial}{\partial z} g_{0}\left(u+u P_{0}(u, v), v+v Q_{0}(u, v)\right)
\end{array}\right.
$$

and $R_{j}(u, v)$ is a linear form of the functions 


$$
\begin{aligned}
& \frac{\partial^{k^{\prime}+l^{\prime}}}{\partial y^{k^{\prime}} \partial z^{l^{\prime}}} f_{j}\left(u+u P_{0}(u, v), v+v Q_{0}(u, v)\right) \\
& \left(j^{\prime}+k^{\prime}+l^{\prime} \leqq j ;\left(j^{\prime}, k^{\prime}, l^{\prime}\right) \neq(0,1,0), \neq(0,0,1) ; 0 \leqq j^{\prime} \leqq j\right)
\end{aligned}
$$

whose coefficients are polynomials in $P_{j}(u, v)$ and $Q_{j}(u, v)$ $\left(1 \leqq j^{\prime}<j\right)$, and the expression $S_{j}(u, v)$ has the same properties as $R_{j}(u, v)$.

Moreover, these differential equations possess a formal solution of the form

$$
\begin{gathered}
P_{0} \sim \sum_{h, l} P_{h l}^{0} U^{k} V^{\imath}, \quad Q_{0} \sim \sum_{h, l} Q_{h l}^{0} U^{k} V^{\imath} \\
\left(P_{h l}^{0}=P_{0, k+1, l}, \quad Q_{k \imath}^{0}=Q_{0, k, l+1}\right)
\end{gathered}
$$

and

$$
P_{j} \sim \sum_{k, l} P_{j k l} U^{k} V^{\iota}, \quad Q_{j} \sim \sum_{k, l} Q_{j k l} U^{k} V^{\imath}
$$

25. Determination of the functions $P_{0}(u, v)$ and $Q_{0}(u, v)$.

1 $^{\circ}$ We consider the differential equations (24.1). These equations can be written in the form

$$
x P^{\prime}=U^{n} V^{n} F(U, V, P, Q), \quad x Q^{\prime}=U^{n} V^{n} G(U, V, P, Q),
$$

where $F(u, v, p, q)$ and $G(u, v, p, q)$ are functions holomorphic and bounded in $(u, v, p, q)$ for

$$
|u|<b, \quad|v|<b, \quad|p|<d, \quad|q|<d .
$$

Moreover, there exists a formal solution of the form

$$
P=f(U, V), \quad Q=g(U, V) .
$$

where $f(U, V)$ and $g(U, V)$ are power series of $U$ and $V$ given by (24.4).

The formal series (III. 1) are a formal solution of (1.1) provided that $(U, V)$ is a solution of (2.3). Now, we choose arbitrarily the initial values $\left(u_{0}, v_{0}\right)$ of the solution $(U, V)$ in such a way that

$$
\left|u^{0}\right|<b, \quad\left|v^{0}\right|<b
$$


and define the solution $(\tilde{U}, \tilde{V})$ of (2.3) such that $\check{U}=u^{0}$ and $\tilde{V}=v^{0}$ at $x=x_{0}$.

Then, we have at once

Assertion 1. If $f(U, V)$ and $g(U, V)$ are a formal solution of (24. 1), then $(f(\tilde{U}, \tilde{V}), g(\tilde{U}, \tilde{V}))$ is a formal solution of the differential equations

(25. 1) $\quad x P^{\prime}=\tilde{U}^{m} \tilde{V}^{n} F(\tilde{U}, \tilde{V}, P, Q), \quad x Q^{\prime}=\tilde{U}^{m} \tilde{V}^{n} G(\tilde{U}, \tilde{V}, P, Q)$.

Conversely, if $f(\tilde{U}, \tilde{V})$ and $g(\tilde{U}, \tilde{V})$ are an actual solution of (25.1), then the expressions $f(U, V)$ and $g(U, V)$ represent $a$ solution of (24.1).

Next, we can assert that

Assertion 2. If $f(\tilde{U}, \tilde{V})$ and $g(\tilde{U}, \tilde{V})$ are a formal solution of (25.1), the power series $f(u, v)$ and $g(u, v)$ must be a formal solution of the partial differential equations

$$
\left\{\begin{array}{l}
u\left(\alpha+\alpha^{\prime} u^{m} v^{n}\right) \frac{\partial p}{\partial u}+v\left(\beta+\beta^{\prime} u^{m} v^{n}\right) \frac{\partial p}{\partial v}=F(u, v, p, q), \\
u\left(\alpha+\alpha^{\prime} u^{m} v^{n}\right) \frac{\partial q}{\partial u}+v\left(\beta+\beta^{\prime} u^{m} v^{n}\right) \frac{\partial q}{\partial v}=G(u, v, p, q) .
\end{array}\right.
$$

Conversely, suppose that $p=f(u, v)$ and $q=g(u, v)$ are the solution of the partial differential equations (25.2). Then, the expressions $f(\tilde{U}, \tilde{V})$ and $g(\tilde{U}, \tilde{V})$ are an actual solution of (25. 1).

By substituting $f(\tilde{U}, \tilde{V})$ and $g(\tilde{U}, \tilde{V})$ for $P$ and $Q$ in (25.1) and dividing both sides of the resulting equations by $\tilde{U}^{m} \tilde{V}^{n}$, we have the relations (25.2), where $u, v, p$ and $q$ must be replaced by $\tilde{U}, \tilde{V}, f(\tilde{U}, \tilde{V})$ and $g(\tilde{U}, \tilde{V})$. However, the initial values $u^{0}$ and $v^{0}$ of the functions $\tilde{U}$ and $\tilde{V}$ can be chosen independently. This fact proves the first part of the assertion. The proof of the remaing part can be carried out by a direct calculation.

Let $(\hat{U}, \hat{V})$ be the holomorphic solution of the differential equations

$$
x \widehat{u}^{\prime}=\widehat{u}\left(\frac{\alpha+\alpha^{\prime} \hat{u}^{m} \widehat{v}^{n}}{m \alpha+n \beta}\right), x \widehat{v}^{\prime}=\widehat{v}\left(\frac{\beta+\beta^{\prime} \hat{u}^{m} \widehat{v}^{n}}{m \alpha+n \beta}\right),
$$


satisfying the initial condition : $\hat{U}=\hat{u}^{0}$ and $\hat{V}=\hat{v}^{0}$ at $x=x_{0}$. Here $\left(x_{0}, \hat{u}^{0}, \hat{v}^{0}\right)$ is an arbitrary point in the domain

$$
|x|<a_{0}, \quad|\widehat{u}|<b_{0}, \quad|\widehat{v}|<b_{0} .
$$

Then, by a direct calculation, we can obtain the following:

Assertion 3. Let $f(u, v)$ and $g(u, v)$ be a formal solution of (25.2). Then, $f(\hat{U}, \hat{V})$ and $g(\hat{U}, \hat{V})$ are a formal solution of the ordinary differential equations

$$
\left\{\begin{array}{l}
x(m \alpha+n \beta) P^{\prime}=F(\hat{U}, \hat{V}, P, Q), \\
x(m \alpha+n \beta) Q^{\prime}=G(\hat{U}, \hat{V}, P, Q) .
\end{array}\right.
$$

Conversely, if $f(\hat{U}, \hat{V})$ and $g(\hat{U}, \hat{V})$ are the solution of (25.4), then the functions $f(u, v)$ and $g(u, v)$ must be a solution of the partial differential equations (25.2).

By summerizing the discussion, we have the following:

Lemma. If the formal series

$$
P \sim \sum_{k, l} P_{k l}^{0} U^{k} V^{\imath}, Q \sim \sum_{k, l} Q_{k l}^{0} U^{k} V^{\imath}
$$

are a formal solution of (24.1), then the formal series

$$
P \sim \sum_{k, l} P_{k l}^{0} \widehat{U}^{k} \hat{V}^{\imath}, Q \sim \sum_{h, l} Q_{k l}^{0} \hat{U}^{k} \hat{V}^{l}
$$

satisfy formally the differential equations (25.4).

If the formal solution (25.5) is uniformly convergent for

$$
|\hat{U}|<b^{\prime \prime}, \quad|\hat{V}|<b^{\prime \prime}
$$

and represents an actual solution of (25.4), then the formal solution (24.4) also converges for $(U, V)$ in the domain (25.6) and becomes a solution of (24.1).

$2^{\circ}$. We can assert that the formal solution (25.5) is uniformly convergent for (25.6) and becomes a solution of (25.4).

Indeed, according to our usual method, we make a transformation of the form

$$
P=\eta+P^{(N)}(\hat{U}, \hat{V}), \quad Q=\zeta+Q^{(N)}(\hat{U}, \hat{V}),
$$

where 


$$
P^{(N)}(u, v)=u+\sum_{k+l<N} P_{k l}^{0} u^{k} v^{l}, Q^{(N)}(u, v)=v+\sum_{k+l<N} Q_{k l}^{0} u^{k} v^{l} .
$$

Then, the system (25.4) can be transformed into

$$
x \eta^{\prime}=\mathfrak{F}(\hat{U}, \hat{V}, \eta, \zeta), x \zeta^{\prime}=\mathbb{S}(\hat{U}, \hat{V}, \eta, \zeta),
$$

where $\mathfrak{\delta}(u, v, \eta, \zeta)$ and $\mathbb{S}(u, v, \eta, \zeta)$ are functions both holomorphic and bounded in $(u, v, \eta, \zeta)$ for

$$
|u|<b_{1}^{\prime}, \quad|v|<b_{1}^{\prime}, \quad|\eta|<d_{1}^{\prime}, \quad|\zeta|<d_{1}
$$

and satisfying there inequality

$$
\max \{|\bar{u}|,|\bar{s}|\} \leqq A(|\eta|+|\zeta|)+B_{N}\left(|u|^{N}+|v|^{N}\right)
$$

and the Lipschitz's condition

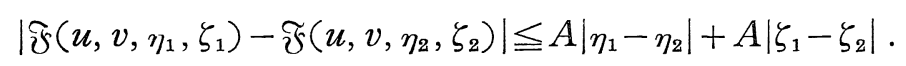

Here $A$ is a positive constant independent of $N$ and $B_{N}$ may depend on $N$. (3) also satisfies the same Lipschitz's condition as $\mho_{\text {. }}$

Let $x_{0}, \hat{u}^{0}$ and $\hat{v}^{0}$ be arbitrary values such that

$$
\left|x_{0}\right|<a^{\prime \prime}, \quad\left|\hat{u}^{0}\right|<b^{\prime \prime}, \quad\left|\hat{v}^{0}\right|<b^{\prime \prime}
$$

and define the functions $\hat{U}$ and $\hat{V}$ as before.

Let $L_{x_{0}}$ be the segment joining the point $x_{0}$ with the origin and let $s$ be the length of this segment measured from the origin to the point $x$. Then, on the path $L_{x_{0}}$ we have the inequalities

$$
\left\{\begin{array}{l}
\frac{d|\dot{U}|}{d s} \geqq \frac{|\alpha| \sin \delta_{0}}{2|m \alpha+n \beta|} \mid \frac{\dot{U} \mid}{|x|}, \\
\frac{d|\hat{V}|}{d s} \geqq \frac{|\beta| \sin \delta_{0}}{2|m \alpha+n \beta|} \frac{|\hat{V}|}{|x|} .
\end{array}\right.
$$

Here $\delta_{0}$ is the same as before.

In fact, since $(\hat{U}, \hat{V})$ is a solution of the differential equations (25.3), we have, for example,

$$
\frac{1}{|\hat{U}|} \frac{d|\hat{U}|}{d s}=\operatorname{Re}\left(\frac{x}{U} \frac{d \hat{U}}{d x} \frac{1}{x} \frac{d x}{d s}\right)=\operatorname{Re}\left(\frac{\alpha+\alpha^{\prime} \hat{U}^{m} \hat{V}^{n}}{m \alpha+n \beta} \frac{1}{x} \frac{d x}{d s}\right) \text {. }
$$

On the other hand, we can assume without loss of generality 
that

$$
\frac{\alpha+\alpha^{\prime} \hat{U}^{m} \hat{V}^{n}}{m \alpha+n \beta}\left|\geqq \frac{|\alpha|}{2|m \alpha+n \beta|},\right| \arg \left(1+\frac{\alpha^{\prime}}{\alpha} \hat{U}^{m} \hat{V}^{n}\right) \mid \leqq 2 \delta_{0}
$$

for (25.6). Therefore, we obtain the first inequality of (25.8), if we notice that

$$
\frac{1}{x} \frac{d x}{d s}=\frac{1}{|x|}
$$

and the inequalities (9.3) are satisfied

Using these inequalities, we can easily verify the following proposition (See, for example, Chapter IV in M. Iwano [3]):

The system (25.7) admits one and only one solution $(\eta, \zeta)$ $=(Y(\hat{U}, \hat{V}), Z(\hat{U}, \hat{V}))$, where $Y(u, v)$ and $Z(u, v)$ are functions holomorphic and bounded in $(u, v)$ for a domain $|u|<b_{N}^{\prime \prime},|v|<b_{N}^{\prime \prime}$ and satisfy there the inequality

$$
\max \{|Y(u, v)|,|Z(u, v)|\} \leqq K_{N}\left(|u|^{N}+|v|^{N}\right),
$$

$K_{N}$ being a certain positive constant.

From this proposition, we can conclude that the formal solution (25.5) is uniformly convergent for the domain (25.6) with $b^{\prime \prime}=\sup b_{N}^{\prime \prime}$ and represents an actual solution of (25.3). We denote by $\left(P_{0}(\hat{U}, \hat{V}), Q_{0}(\hat{U}, \hat{V})\right)$ such a solution.

By the discussion in $1^{\circ}$, the functions $P_{0}(u, v)$ and $Q_{0}(u, v)$ thus determined satisfy the conditions in question.

26. Determination of the functions $P_{j}(u, v)$ and $Q_{j}(u, v)$.

We consider the linear differential equations (24.2.j) and the corresponding formal solutions (24.5.j).

I. The functions $P_{1}(u, v)$ and $Q_{1}(u, v)$.

The differential equations satisfied by $P_{1}(U, V)$ and $Q_{1}(U, V)$ are given by the equations (24.2.1):

$$
\begin{gathered}
x P^{\prime}=-P+C_{11} P+C_{12} Q+R_{1}, x Q^{\prime}=-Q+C_{21} P+C_{22} Q+S_{1}, \\
C_{i j} \equiv C_{i j}(U, V), R_{1} \equiv R_{1}(U, V), S_{1} \equiv S_{1}(U, V) .
\end{gathered}
$$


The functions $U$ and $V$ are the same as those defined in $\mathbb{1 4}$. As we have just seen, the functions $P_{0}(u, v)$ and $Q_{0}(u, v)$ are holomorphic and bounded in $(u, v)$ for

$$
|u|<b^{\prime}, \quad|v|<b^{\prime} .
$$

Hence, by definition of the functions $C_{i j}(u, v), R_{1}(u, v), S_{1}(u, v)$, these functions are also holomorphic and bounded in $(u, v)$ for the domain (26.1) and we have

$$
C_{i j}(0,0)=0 .
$$

Moreover, the equations above admit a formal solution

$$
P \sim \sum_{k, l} P_{1 k l} U^{k} V^{\imath}, \quad Q \sim \sum_{k, l} Q_{1 k l} U^{k} V^{\imath} .
$$

We introduce an auxiliary variable $w$ by

$$
w=U^{m} V^{n} .
$$

As we have already seen in $11, x, U$ and $V$ are considered as functions of $w$. Let $\tilde{x}(w), \tilde{U}(w)$ and $\tilde{V}(w)$ be these functions. Then, they are the solution of the differential equations

$$
\left\{\begin{array}{l}
w^{2} X(w) \frac{d x}{d w}=x, \\
w X(w) \frac{d u}{d w}=u\left(\alpha+\alpha^{\prime} w\right), \quad w X(w) \frac{d v}{d w}=v\left(\beta+\beta^{\prime} w\right), \\
X(w) \equiv m \alpha+n \beta+\left(m \alpha^{\prime}+n \beta^{\prime}\right) w,
\end{array}\right.
$$

such that $\tilde{x}=x_{0}, \tilde{U}=u^{0}$ and $\tilde{V}=v^{0}$ at $w=w^{0}\left(\equiv u^{0 m} v^{0 n}\right)$. Here, $\left(x_{0}\right.$, $\left.u^{0}, v^{0}\right)$ is an arbitrary point in the domain (14.3).

By a direct calculation, we can prove the following:

Lemma. The formal series

$$
P \sim \sum P_{1 k l} \tilde{U}^{k} \tilde{V}^{\imath}, \quad Q \sim \sum Q_{1 k l} \tilde{U}^{k} \tilde{V}^{\imath}
$$

are a formal solution of the differential equations

(26. 5) $\begin{array}{r}w^{2} X(w) \frac{d P}{d w}=-P+C_{11}(\tilde{U}, \hat{V}) P+C_{12}(\tilde{U}, \tilde{V}) Q \\ +R_{1}(\tilde{U}, \tilde{V}),\end{array}$ 


$$
\begin{array}{r}
w^{2} X(w) \frac{d Q}{d w}=-Q+C_{21}(\tilde{U}, \tilde{V}) P+C_{22}(\tilde{U}, \tilde{V}) Q \\
+S_{1}(\tilde{U}, \tilde{V}) .
\end{array}
$$

Conversely, let $f(\tilde{U}, \tilde{V})$ and $g(\tilde{U}, \tilde{V})$ be the solution of (26.5). Then, the functions $f(U, V)$ and $g(U, V)$ are a solution of the equations (24.2.1).

In order to invetigate the analytical meaning of the formal solution (26.4), we put

$$
P^{(N)}(u, v)=\sum_{k+l<N} P_{1 k l} u^{k} v^{z}, \quad Q^{(N)}(u, v)=\sum_{k+l<N} Q_{1 k l} u^{k} v^{l}
$$

and apply a transformation of the form

$$
P=p+P^{(N)}(\tilde{U}, \tilde{V}), \quad Q=q+Q^{(N)}(\tilde{U}, \tilde{V}) .
$$

Then, the transformed differential equations can be written in the form

$$
\left\{\begin{aligned}
w^{2} \frac{d p}{d w}=-\frac{1}{m \alpha+n \beta} p & +F_{11}(\tilde{U}, \tilde{V}) p \\
& +F_{12}(\tilde{U}, \tilde{V}) q+R_{1}^{*}(\tilde{U}, \tilde{V}), \\
w^{2} \frac{d q}{d w}=-\frac{1}{m \alpha+n \beta} q & +F_{21}(\tilde{U}, \tilde{V}) p \\
& +F_{22}(\tilde{U}, \tilde{V}) q+S_{1}^{*}(\tilde{U}, \tilde{V}) .
\end{aligned}\right.
$$

Here, the functions $F_{i j}(u, v), R_{1}^{*}(u, v)$ and $S_{1}^{*}(u, v)$ are holomorphic and bounded in $(u, v)$ for $(26.1)$ and vanish at $(0,0)$. If we notice that the power series

$$
p \sim \sum_{k+l \geqq N} P_{1 k l} \tilde{U}^{k} \tilde{V}^{l}, \quad q \sim \sum_{k+l \geqq N} Q_{1 k l} \tilde{U}^{k} \tilde{V}^{l}
$$

are a formal solution of (26.7), it is not difficult to verify that inequalities of the form

$$
\left|F_{i j}(u, v)\right| \leqq A, \quad \max \left\{\left|R_{1}^{*}(u, v)\right|,\left|S_{1}^{*}(u, v)\right|\right\} \leqq B_{N}\left(|u|^{N}+|v|^{N}\right)
$$

are satisfied for (26.1). Here $A$ is a positive constant independent of $N$ and $B_{N}$ may depend on $N$.

Put 


$$
p=\eta \exp \Lambda(w), q=\zeta \exp \Lambda(w), \quad \Lambda(w) \equiv \frac{1}{(m \alpha+n \beta) w} .
$$

Then, we have

$$
\left\{\begin{array}{l}
\eta^{\prime}=w^{-2}\left[F_{11} \eta+F_{12} \zeta+R_{1}^{*} \exp (-\Lambda(w))\right], \\
\zeta^{\prime}=w^{-2}\left[F_{21} \eta+F_{22} \zeta+S_{1}^{*} \exp (-\Lambda(w))\right] .
\end{array} \quad\left({ }^{\prime}=\frac{d}{d w}\right)\right.
$$

The function $\exp (-j \Lambda(w))$ has the same asymptotic behavior as the function $\check{x}(w)^{j}$, where $j$ is a positive integer. Hence, by Lemma 3.2 in 11 we have the inequalities

$$
\frac{d}{d s} e^{-\jmath \operatorname{Re} \Lambda(w)} \geqq \frac{j \sin \delta_{0}}{2|m \alpha+n \beta|} \frac{1}{|w|^{2}} e^{-j \operatorname{Re} \Lambda(w)}, \quad\left(\delta_{0}>0\right)
$$

and

$$
\begin{aligned}
\frac{d}{d s}\left\{\left(|\hat{U}|^{N}\right.\right. & \left.\left.+|\bar{V}|^{N}\right) e^{-j \operatorname{Re} \Lambda(w)}\right\} \\
& \geqq \frac{j \sin \delta_{0}}{2|m \alpha+n \beta|} \frac{1}{|w|^{2}}\left(|\tilde{U}|^{N}+|\bar{V}|^{N}\right) e^{-j \operatorname{Re} \Lambda(w)}
\end{aligned}
$$

on the curve $\Gamma_{w^{0}}^{*}$ defined in 10 , if the point $\left(u^{0}, v^{0}\right)$ is contained in the domain

$$
|u|<b_{0}, \quad|v|<b_{0}, \quad u^{m} v^{n} \in \mathcal{D}\left(c_{0}\right) .
$$

Using these inequalities, we can assert that: The system (26.10) admits one and only one solution $\eta=Y_{N}(\tilde{U}, \tilde{V}), \quad \zeta=$ $Z_{N}(\hat{U}, \tilde{V})$ such that $Y_{N}(u, v)$ and $Z_{N}(u, v)$ are functions holomorphic and bounded in $(u, v)$ for a domain $|u|<b_{\Lambda}^{0},|v|<b_{N}^{0}$, $u^{m} v^{n} \in \mathfrak{D}\left(c_{*}^{0}\right)$ and satisfying there the inequalities

$$
\max \left\{\left|Y_{N}(u, v)\right|,\left|Z_{N}(u, v)\right|\right\} \leqq K_{N}\left(|u|^{N}+|v|^{N}\right) e^{-\mathrm{Re} \Lambda(v)},
$$

where $w=u^{m} v^{n}$ and $K_{N}$ is a certain positive constant.

The proof of this assertion is almost exactly the same as that of Theorem 4 in 42 in M. Iwano [2], if we notice that we can assume without loss of generality that

$$
4 A|m \alpha+n \beta| \leqq \sin \delta_{0} .
$$

We omit therefore the proof. (The present case is not convergent!) 
By virtue of this assertion, we see that the differential equations (26.5) admit a solution $P=P_{1}(\tilde{U}, \tilde{V}), Q=Q_{1}(\tilde{U}, \tilde{V})$, where $P_{1}(u, v)$ and $Q_{1}(u, v)$ are functions holomorphic and bounded and asymptotically developable in the power series (26.4), where we replace $(\tilde{U}, \tilde{V})$ by $(u, v)$, for the domain (26.11) with $b_{0}=\sup b_{N}^{0}, \quad c_{0}=\sup c_{\mathrm{N}}^{0} . \quad$ Then, by the lemma, $P_{1}(U, V)$ and $Q_{1}(U, V)$ are a solution of (24.2.1) with the desired properties.

II. The functions $P_{j}(u, v)$ and $Q_{j}(u, v)(j \geqq 2)$.

The method to determine these functions is quite the same as before. The differential equations (26.5) must be replaced by

(26. 12. $j)$

$$
\begin{gathered}
\left\{\begin{array}{l}
w^{2} X(w) \frac{d P}{d w}=-j P+\check{C}_{11} P+\check{C}_{12} Q+\tilde{R}_{j}, \\
w^{2} X(w) \frac{d Q}{d w}=-j Q+\check{C}_{21} P+\check{C}_{22} Q+\tilde{S}_{j},
\end{array}\right. \\
\tilde{C}_{i j}=C_{i j}(\check{U}, \tilde{V}), \check{R}_{j}=R_{j}(\check{U}, \check{V}), \tilde{S}_{j}=S_{j}(\check{U}, \tilde{V}) .
\end{gathered}
$$

Here, the functions $R_{j}(u, v)$ and $S_{j}(u, v)$ admit not convergent but asymptotic expansions in powers of $u$ and $v$ as $(u, v)$ tends to 0 in the domain (26.11).

Observe, by the lemma, that this system admits a formal solution similar to $(24.5 . j)$ :

$$
P \sim \sum_{k, l} P_{j_{k l}} \tilde{U}^{k} \tilde{V}^{l}, \quad Q \sim \sum_{h, l} Q_{j_{k l}} \hat{U}^{k} \tilde{V}^{l} .
$$

We make apply succesively transformations similar to (26.6) and (26.9), where $\Lambda(w)$ must be replaced by $j \Lambda(w)$.

Repeating word by word the discussion in $\mathbb{I}$, we can determine the functions $P_{j}(u, v)$ and $Q_{j}(u, v)$ in such a way that the expressions $P_{j}(\check{U}, \check{V})$ and $Q_{j}(\check{U}, V)$ are a solution of the system (26.12.j) admitting asymptotic expansions of the form (26.13. $j$ ) for $(U, V)$ in the domain (26.11).

Then, by the Lemma, the functions $P_{j}(u, v)$ and $Q_{j}(u, v)$ thus determined satisfy the conditions stated in Theorem 4.3.1. 
Thus, Theorem 4.3.1 has been completely verified for Case A.

Remark. As can be easily verified, in the case when $m=0$ (or $n=0$ ), the domain (26.11), where the functions $P_{j}(u, v)$ and $Q_{j}(u, v)(j \geqq 1)$ are defined, contains the point $u=0$ (or the point $v=0)$ as an inner point.

We can assert that these functions are developable in uniformly convergent power series of $u$ (or $v$ ) according as $m=0$ (or $n=0$ ).

We shall give a brief sketch of the proof for $j=1$ in the case $m=0$.

Let $\left(u^{0}, v^{0}\right)$ be an arbitrary point in the domain $\left(14.3^{\prime \prime}\right)$ and define the functions $\tilde{U}$ and $\tilde{V}$ as before. We rearrange the formal power series (26.4) in the form of a single power series of $U$ as follows :

$$
P \sim \sum_{l} p_{l}(\tilde{V}) \tilde{U}^{\imath}, \quad Q \sim \sum_{l} q_{l}(\tilde{V}) \tilde{U}^{l},
$$

where

$$
p_{l}(v) \sim \sum_{k} P_{1 k l} v^{k}, \quad q_{l}(v) \sim \sum_{k} Q_{1 k l} v^{k} .
$$

Insert the formal solution (26.4)* into (26.5) and observe that

$$
\begin{aligned}
w^{2} X & (w) \frac{d}{d w}\left\{p_{l}(\tilde{V}) \tilde{U}^{l}\right\} \\
& =w\left\{\frac{d p_{l}(\tilde{V})}{d \tilde{V}} \cdot w X(w) \frac{d \tilde{V}}{d w}+p_{l}(\tilde{V}) \cdot w X(w) \frac{d \tilde{U}}{d w} \tilde{U}^{-1}\right\} \tilde{U}^{l} \\
& =\left\{\tilde{V}^{n+1}\left(\beta+\beta^{\prime} \tilde{V}^{n}\right) \frac{d p_{l}(\tilde{V})}{d \tilde{V}}+\tilde{V}^{n}\left(\alpha+\alpha^{\prime} \tilde{V}^{n}\right) p_{l}(\tilde{V})\right\} \tilde{U}^{l}
\end{aligned}
$$

The resulting equations are formal power series of $\tilde{U}$ whose coefficients are functions of $\check{V}$. Equating the coefficients of the terms $\tilde{U}^{v}$ of these equations and replacing $\tilde{V}$ by $v$, we obtain the linear differential equations satisfied by the functions $p_{l}(v)$ and $q_{l}(v)$. If we pick up their leading terms only, they are 
written in the form

$$
\left\{\begin{array}{l}
v^{n+1}\left(\beta+\beta^{\prime} v^{n}\right) p_{l}^{\prime}=-p_{l}+\cdots \\
v^{n+1}\left(\beta+\beta^{\prime} v^{n}\right) q_{l}^{\prime}=-q_{l}+\cdots
\end{array}\right.
$$

Therefore, the functions $p_{l}(v)$ and $q_{l}(v)$ are uniquely determined as solutions of these differential equations in such a way that they admit asymptotic expansions in the form (26.14. $l$ ) for

$$
|n \arg v+\arg \beta|<\frac{3 \pi}{2}, \quad 0<|v|<b^{\prime} .
$$

Then, by the discussion in $\mathbb{I}$, we see that there exists a solution $\left(P_{1}(\tilde{U}, \tilde{V}), Q_{1}(\tilde{U}, \tilde{V})\right)$ of (26.5) which admit asymptotic expansions in the form $(26.4)^{*}$ as $(\tilde{U}, \tilde{V})$ tends to 0 in the domain

$$
|u|<b_{0}, \quad v^{n} \in \mathfrak{D}\left(c_{0}\right) .
$$

However, the point $u=0$ is an inner point of this domain. From this it follows that the formal solution (26.4)* is uniformly convergent for $(\tilde{U}, \tilde{V})$ in the domain above.

This proves the assertions stated in the Remark following Theorem 4.3.1.

Section IV. Proof of Theorems 4.3.2 and 4.3.3.

\section{Preliminaries.}

Since the discussion for Theorem 4.3. 3 can be carried out in quite a similar way, we discuss the proof of Theorem 4.3.2 only. And, we must discuss Case A and Case B separately. But, since the discussion for Case $B$ is almost similar to that for Case A, we consider the Case A only.

If we differentiate the formal solution (III.2) term by term, then we have

$$
\left\{\begin{array}{c}
x y^{\prime} \sim \sum_{l=0}^{\infty}\left(x \frac{d P_{l}}{d x}+l P_{l} \frac{x V^{\prime}}{V}\right) V^{\imath}, \\
x z^{\prime} \sim \sum_{l=0}^{\infty}\left(x \frac{d Q_{l}}{d x}+l Q_{l} \frac{x V^{\prime}}{V}\right) V^{\imath},
\end{array}\right.
$$


where

$$
x \frac{d P_{l}}{d x} \equiv x \frac{\partial P_{l}}{\partial x}+\frac{\partial P_{l}}{\partial U^{-}} \cdot x U^{\prime}, x \frac{d Q_{l}}{d x} \equiv x \frac{\partial Q_{l}}{\partial x}+\frac{\partial Q_{l}}{\partial U} \cdot x U^{\prime}
$$

Thus we have

$$
\left\{\begin{array}{l}
f(x, y, z) \sim \sum_{l=0}^{\infty}\left(x \frac{d P_{l}}{d x}+l U^{m} V^{n}\left(\beta+\beta^{\prime} U^{m} V^{n}\right) P_{l}\right) V^{l} \\
g(x, y, z) \sim \sum_{l=0}^{\infty}\left(x \frac{d Q_{l}}{d x}+l U^{m} V^{n}\left(\beta+\beta^{\prime} U^{m} V^{n}\right) Q_{l}\right) V^{l}
\end{array}\right.
$$

where $f(x, y, z)$ and $g(x, y, z)$ are functions holomorphic and bounded in $(x, y, z)$ in a neighborhood of $(0,0,0)$.

Observe that, in the case when $n>0$, the expression $x U^{\prime}$ depends on both of $U$ and $V$, while, in the case when $n=0$, it depends on $U$ only.

Substituting the formal series (III.2) for $y$ and $z$ appearing in the left-hand members of (27.3), we obtain formal identities between formal power series of $V$ whose coefficients are functions of $x$ and $U$. Comparing the coefficients of the terms $V^{\imath}(l \geqq 0)$ of such formal identities, we can find the relations which the coefficients $P_{l}(x, U)$ and $Q_{l}(x, U)$ must satisfy. A simple calculation shows us that these relations are given by the following differential equations:

Case when $n>0$ :

$$
x \frac{\partial P_{0}}{\partial x}=f\left(x, P_{0}, Q_{0}\right), \quad x \frac{\partial Q_{0}}{\partial x}=g\left(x, P_{0}, Q_{0}\right)
$$

and

$$
\left\{\begin{array}{l}
x \frac{\partial P_{l}}{\partial x}=C_{11}(x, U) P_{\imath}+C_{12}(x, U) Q_{\imath}+R_{l}(x, U) \\
x \frac{\partial Q_{l}}{\partial x}=C_{21}(x, U) P_{\imath}+C_{22}(x, U) Q_{\imath}+S_{l}(x, U)
\end{array}\right.
$$

with

$$
C_{11}(x, u)=\frac{\partial}{\partial y} f\left(x, P_{0}(x, u), Q_{0}(x, u)\right)
$$




$$
\begin{aligned}
& C_{12}(x, u)=\frac{\partial}{\partial z} f\left(x, P_{0}(x, u), Q_{0}(x, u)\right), \\
& C_{21}(x, u)=\frac{\partial}{\partial y} g\left(x, P_{0}(x, u), Q_{0}(x, u)\right), \\
& C_{22}(x, u)=\frac{\partial}{\partial z} g\left(x, P_{0}(x, u), Q_{0}(x, u)\right), \\
& R_{l}(x, u)=-\alpha u^{m+1} \frac{\partial}{\partial u} P_{l-n}(x, u)-\alpha^{\prime} u^{2 m+1} \frac{\partial}{\partial u} P_{l-2 n}(x, u) \\
&-(l-n) \beta u^{m} P_{l-n}(x, u)-(l-2 n) \beta^{\prime} u^{2 m} P_{l-2 n}(x, u) \\
&+\Re_{l}(x, u), \\
& S_{l}(x, u)=-\alpha u^{m+1} \frac{\partial}{\partial u} Q_{l-n}(x, u)-\alpha^{\prime} u^{2 m+1} \frac{\partial}{\partial u} Q_{l-2 n}(x, u) \\
&-(l-n) \beta u^{m} Q_{l-n}(x, u)-(l-2 n) \beta^{\prime} u^{2 m} Q_{l-2 n}(x, u) \\
&+\Im_{l}(x, u) .
\end{aligned}
$$

Here $\mathfrak{\Re}_{l}(x, u)$ (or $\left.\bigodot_{l}(x, u)\right)$ is a linear form of the partial derivatives $\frac{\partial^{k^{\prime}+l^{\prime}}}{\partial y^{k} \partial z^{l^{\prime}}} f\left(x, P_{0}(x, u), Q_{0}(x, u)\right)$ (or $\frac{\partial^{k^{\prime}+l^{\prime}}}{\partial y^{k^{\prime}} \partial z^{l^{\prime}}} g\left(x, P_{0}(x, u), Q_{0}\right.$ $(x, u)))$ for $k^{\prime}+l^{\prime} \leqq l ;\left(k^{\prime}, l^{\prime}\right) \neq(1,0), \neq(0,1)$, whose coefficients are polynomials of the functions $P_{l^{\prime}}(x, u)$ and $Q_{r^{\prime}}(x, u)$ for $1 \leqq l^{\prime}<l$.

Moreover, as we have already seen, these partial differential equations admit a formal solution of the form

$$
\left\{\begin{array}{l}
P_{l} \sim \delta_{l 0} U+U P_{l}^{(2)}(U)+\sum_{j=1}^{\infty} P_{\jmath l}^{(2)}(U) x^{j}, \\
Q_{l} \sim \delta_{l 1}+Q_{l-1}^{(2)}(U)+\sum_{j=1}^{\infty} Q_{\jmath l}^{(2)}(U) x^{j},
\end{array} \quad(l=0,1, \cdots),\right.
$$

where the coefficients $P_{l}^{(2)}(u), Q_{l-1}^{(2)}(u), P_{j l}^{(2)}(u)$ and $Q_{j l}^{(2)}(u)$ are the same functions as those that appeared in Theorem 4.2.2. Consequently, they are functions holomorphic and bounded in a circle $|u|<b_{0}^{\prime}$.

Case when $n=0$ :

In this case, the partial differential equations (27.4) and (27. 5. l) must be replaced by:

$$
x P_{0}^{\prime}=f\left(x, P_{0}, Q_{0}\right), \quad x Q_{0}^{\prime}=g\left(x, P_{0}, Q_{0}\right)
$$

and 
$(27.8 . l)\left\{\begin{aligned} & x P_{l}^{\prime}=\left(C_{11}(x, U)-l \beta U^{m}-l \beta^{\prime} U^{2 m}\right) \cdot P_{l}+C_{12}(x, U) \cdot Q_{l} \\ &+\Re_{l}(x, U), \\ & x Q_{l}^{\prime}=C_{21}(x, U) \cdot P_{l}+\left(C_{22}(x, U)-l \beta U^{m}-l \beta^{\prime} U^{2 m}\right) \cdot Q_{l}+\bigodot_{l}(x, U) .\end{aligned}\right.$

Moreover, these differential equations admit a formal solution of the same form as $(27.6 . l)$, where the coefficients $P_{\jmath l}^{(2)}(u)$ and $Q_{\jmath l}^{(2)}(u)$ must be replaced by functions holomorphic, bounded and asymptotically developable in powers of $u$ for

$$
|m \arg u+\arg \alpha|<\frac{3 \pi}{2}, 0<|u|<b_{0}^{\prime} .
$$

28. Determination of the coefficients $P_{l}(x, u)$ and $Q_{l}(x, u)$ for $l \geqq 0$. The formal solution (27.6.l) can be written in the form

$$
P_{l} \sim \sum_{j=0}^{\infty} p_{l, j}(U) x^{j}, \quad Q_{l} \sim \sum_{j=0}^{\infty} q_{l, j}(U) x^{j}
$$

Then, by Theorem 4.2 .2 , the coefficients $p_{l, j}(u)$ and $q_{l, j}(u)$ are functions holomorphic and bounded in a circle $|u|<b_{0}^{\prime}$ or functions holomorphic, bounded and asymptotically developable in powers of $u$ for an angular region of the form (27.9) according as we have $(n>0$ or $n=0$ and $j=0)$ or $(n=0$ and $j \geqq 1)$.

The partial differential equations (27.4) and (27.5.l) can be considered as ordinary differential equations if we regard $u$ as a parameter. Therefore, they are ordinary differential equations with a singularity $x=0$ similar to the regular singular point of the linear system. It is known that, if its formally holomorphic solution exists, it is actually holomorphic. Hence, the formal solution (28.1.l) is uniformly convergent for

$$
|x|<a_{0}^{\prime}, \quad|U|<b_{0}^{\prime}
$$

and represents a solution of the corresponding differential equations. We denote by $P_{l}(x, U)$ and $Q_{l}(x, U)$ such solutions. Then, the functions $P_{l}(x, u)$ and $Q_{l}(x, u)$ are clearly developable in uniformly convergent power series of $x$ and $u$ for the case 
when $n>0$.

In the case when $n=0$, we can assert that the formal solution (28.1.l) is uniformly convergent for

$$
|x|<a_{0}^{\prime}, \quad|m \arg U+\arg \alpha|<\frac{3 \pi}{2}, \quad 0<|U|<b_{0}^{\prime}
$$

and represents a solution of the corresponding differential equations.

For the proof of this assertion, we have to establish a lemma similar to the Lemma 3.2 in $\mathbf{1 1}$ for solutions of the differential equation

$$
x u^{\prime}=u^{m+1}\left(\alpha+\alpha^{\prime} u^{m}\right) .
$$

We notice that, since $n=0$, the function $U$ appearing in the differential equations (27.7) and (27.8. $l$ ) and their formal solutions (28.1. $l$ ) is a solution of (28.4).

Let $\widehat{D}\left(c_{0}^{\prime}\right)$ be the set of points $w$ satisfying the inequalities

$$
\hat{\Theta}_{-}<\arg w<\widehat{\Theta}_{+}, \quad 0<|w|<\hat{d}\left(\arg w, c_{0}^{\prime}\right),
$$

where

$$
\widehat{\Theta}_{ \pm}=-\arg \alpha \pm \frac{3 \pi}{2} \mp 2 \delta_{0}
$$

and the function $\hat{d}(\varphi, c)$ has the property similar to $d(\varphi, c)$ appearing in 14. We denote by $U=\hat{U}\left(x, x_{0}, u^{0}\right)$ the holomorphic solution of (28.4) satisfying the initial condition $U=u^{0}$ at $x=x_{0}$. Here, $\left(x_{0}, u^{0}\right)$ is an arbitrary point in the domain

$$
|x|<a_{0}^{\prime}, \quad u^{m} \in \hat{\mathfrak{S}}\left(c_{0}^{\prime}\right) .
$$

This solution can be parametrically represented as follows

$$
U=w^{\frac{1}{m}}, \quad x=\hat{x}(w),
$$

where $\hat{x}(w)$ is the holomorphic solution of

$$
w^{2} \frac{d x}{d w}=\frac{x}{m \alpha+m \alpha^{\prime} w}
$$


such that $x=x_{0}$ at $w=w^{0}\left(\equiv\left(u^{0}\right)^{m}\right)$. Moreover, the elimination of $w$ from these two relations yields $U=\hat{U}\left(x, x_{0}, u^{0}\right)$ provided that the point $\left(x_{0}, u^{0}\right)$ is in the domain (28.5) (See 11).

The lemma which we intend to establish can be stated as follows :

Lemma. Let $w^{0}$ be an arbitrary value such that

$$
w^{0} \in \hat{\mathfrak{D}}\left(c_{0}^{\prime}\right) \text {. }
$$

Then, there exists a curve $\hat{\Gamma}_{w^{0}}^{*}$, which starts from the point $w^{0}$ and approaches 0 in the sector $|\arg w+\arg \alpha| \leqq \frac{\pi}{2}-2 \delta_{0}$, such that, on the curve $\hat{\Gamma}_{w^{0}}^{*}$, we have the inequality

$$
\frac{d|\hat{x}(w)|}{d s} \geqq \frac{\sin \delta_{0}}{2 m|\alpha|} \frac{1}{|w|^{2}}|\widehat{x}(w)| .
$$

Here, $s$ is the length of of the curve $\hat{\Gamma}_{w 0}^{*}$ measured from the origin to the point $w^{0}$.

The proof of this lemma can be carried out in quite a similar way as that for Lemma 3.2 if we replace $\Theta_{-}$and $\Theta_{+}$by $\widehat{\Theta}_{-}$and $\hat{\Theta}_{+}$in the formula (12.24).

By virtue of this lemma, the proof of our assertion mentioned above is almost exactly the same as that of Theorem 5.3. 1 in Chapter $V$. We therefore omit the proof of the assertion for $n=0$.

Remark. For Case B, the expression $x U^{\prime}$ depends on both of $U$ and $V$ even if $n=0$. Therefore, the differential equations (27.7) and (27.8.l) must be replaced by partial differential equations. We have, for example,

$$
\left\{\begin{array}{l}
x \frac{\partial P_{0}}{\partial x}+\mu U^{m+1}\left(\gamma+\gamma_{0} U^{m}\right) \frac{\partial P_{0}}{\partial U}=f\left(x, P_{0}, Q_{0}\right), \\
x \frac{\partial Q_{0}}{\partial x}+\mu U^{m+1}\left(\gamma+\gamma_{0} U^{m}\right) \frac{\partial Q_{0}}{\partial U}=g\left(x, P_{0}, Q_{0}\right)
\end{array}\right.
$$

and there exists a formal solution

$$
P_{0} \sim \sum p_{0, j}(U) x^{j}, \quad Q_{0} \sim \sum q_{0, j}(U) x^{j} .
$$


Let $\hat{U} \equiv \hat{U}\left(x, x_{0}, u^{0}\right)$ be the holomorphic solution of the differential equation

$$
x \frac{d \hat{u}}{d x}=\mu \hat{u}^{m+1}\left(\gamma+\gamma_{0} \hat{u}^{n l}\right)
$$

satisfying the initial condition $\hat{U}=u^{0}$ at $x=x_{0}$. Then, we can easily verify that the formal series

$$
\hat{P}_{0} \sim \sum p_{0, j}(\hat{U}) x^{j}, \quad Q_{0} \sim \sum q_{0, j}(\hat{U}) x^{j}
$$

are a formal solution of the differential equations

$$
x \frac{d \hat{P}_{0}}{d x}=f\left(x, \hat{P}_{0}, \widehat{Q}_{0}\right), \quad x \frac{d \widehat{Q}_{0}}{d x}=g\left(x, \hat{P}_{0}, \hat{Q}_{0}\right) .
$$

Hence, the proof of the uniform convergence of this formal solution is the same as that for Case A and $n=0$.

\section{Part III. The analytical meaning.}

This part will be devoted to the investigation of an analytical meaning of formal solutions of diverse types which have been already studied in Part II.

Chapter V. Convergence or asymptotic development

\section{Main Results.}

In the preceding chapter we have obtained formal solutions of the system (1.1) of diverse types, namely (I) (Theorem 4.1), (II. 1) (Theorem 4.2.1), (II. 2) (Theorem 4.2.2), (II. 3) (Theorem 4.2.3), (III. 1) (Theorem 4.3.1), (III. 2) (Theorem 4.3.2), (III. 3) (Theorem 4.3.3).

In this chapter, we shall study an analytical meaning of these formal solutions. Let $U$ and $V$ be the same functions as those that appeared in 14 in Chapter IV and let $\mathcal{D}(c)$ be the angular region defined by (14.2).

The results which we intend to establish can be stated as 
follows :

Theorem 5.1. There exists a solution $(y, z)=(\Phi(x, U, V)$, $\Psi(x, U, V))$ of the system (1.1) such that $\Phi(x, u, v)$ and $\Psi(x, u, v)$ are functions holomorphic and bounded in $(x, u, v)$ for

$$
|x|<a_{0}^{\prime \prime}, \quad|u|<b_{0}^{\prime \prime}, \quad|v|<b_{0}^{\prime \prime}, \quad u^{m} v^{n} \in \mathfrak{D}\left(c_{0}^{\prime \prime}\right)
$$

and, moreover, admitting asymptotic expansions of the form of (I) :

$$
\begin{cases}\Phi(x, u, v) \approx u+\sum_{j, k, l}^{(2)} P_{j k l} x^{j} u^{k} v^{l}, & P_{00 l}=0 \\ \Psi(x, u, v) \approx v+\sum_{j, k, l}^{(2)} Q_{j k l} x^{j} u^{k} v^{l}, & Q_{0 k 0}=0\end{cases}
$$

as $(x, u, v)$ tends to 0 in the domain (29.1), where $a_{0}^{\prime \prime}, b_{0}^{\prime \prime}$ and $c_{0}^{\prime \prime}$ are suitably chosen positive constants.

In the case when $n=0$, the domain (29.1) must be replaced by

$$
|x|<a_{0}^{\prime \prime}, \quad|v|<b_{0}^{\prime \prime}, \quad u^{m} \in \mathfrak{D} c_{0}^{\prime \prime} .
$$

In the case when $m=0$, the domain (29.1) must be replaced by

$$
|x|<a_{0}^{\prime \prime}, \quad|u|<b_{0}^{\prime \prime}, \quad v^{n} \in \mathfrak{D} c_{0}^{\prime \prime} .
$$

Remark. The point $(u, v)$ satisfying the inequalities (29.1) is located on the product space of the Riemann surfaces of $\log u$ and $\log v$.

The asymptotic expansion $\left(\mathrm{I}^{\prime}\right)$ means that, for any positive integer $N$, we have an inequality of the form

$$
\left|\Phi(x, u, v)-u-\sum_{j+k+l<N} P_{j k l} x^{j} u^{k} v^{l}\right| \leqq K_{N}\left(|x|^{N}+|u|^{N}+|v|^{N}\right)
$$

for any $(x, u, v)$ in $(29.1), K_{N}$ being a certain positive constant which may depend on $N$.

Theorem 5.2.1. The differential equations (1.1) have a solution $(y, z)=(\Phi(x, U, V), \Psi(x, U, V))$, where $\Phi(x, u, v)$ and $\Psi(x, u$, $v$ ) are functions holomorphic and bounded in $(x, u, v)$ for (29.1) and, moreover, asymptotically developable in the form of (II. 1): 
(II. $\left.1^{\prime}\right)$

$$
\left\{\begin{array}{l}
\Phi(x, u, v) \approx u+\sum_{h, l} P_{k l}^{(1)}(x) u^{k} v^{l}, \\
\Psi(x, u, v) \approx v+\sum_{h, l} Q_{k l}^{(1)}(x) u^{k} v^{l},
\end{array}\right.
$$

uniformly valid for $|x|<a_{0}^{\prime \prime}$, as $u$ and $v$ tend to 0 in the domain (29.1), where $P_{h l}^{(1)}(x)$ and $Q_{k l}^{(1)}(x)$ are holomorphic and bounded functions of $x$ for $|x|<a_{0}^{\prime}\left(a_{0}^{\prime} \geqq a_{0}^{\prime \prime}\right)$.

Remark. The asymptotic development means that, for any positive integer $N$, an inequality of the form

(II. $\left.1^{\prime \prime}\right) \quad\left|\Phi(x, u, v)-u-\sum_{k+l<N} P_{h l}^{(1)}(x) u^{k} v^{z}\right| \leqq K_{N}\left(|u|^{N}+|v|^{N}\right)$

is uniformly valid for (29.1). Of course, $K_{N}$ depends on $N$ only.

Theorem 5.2.2. The system (1.1) possesses a solution $(y, z)$ $=(\Phi(x, U, V), \Psi(x, U, V))$, where $\Phi(x, u, v)$ and $\Psi(x, u, v)$ are functions holomorphic and bounded in $(x, u, v)$ for (29.1) and admitting asymptotic expansions of the form of (II. 2):

$$
\left\{\begin{array}{l}
\Phi(x, u, v) \approx u\left(1+\sum_{l=0}^{\infty} P_{l}^{(2)}(u) v^{l}\right)+\sum_{j \geqq 1, l \geqq 0} P_{j l}^{(2)}(u) x^{j} v^{l} \\
\Psi(x, u, v) \approx v\left(1+\sum_{l=0}^{\infty} Q_{l}^{(2)}(u) v^{l}\right)+\sum_{j \geqq 1, l \geqq 0} Q_{j l}^{(2)}(u) x^{j} v^{l}
\end{array}\right.
$$

uniformly valid for $|u|<b_{0}^{\prime \prime}$, as $x$ and $v$ tend to 0 in the domain (29.1), where the coefficients are the same functions as those appearing in Theorem 4.2.2.

If $n=0$, the formal series (II. $2^{\prime}$ ) are uniformly convergent in a domain (29.2) so that, if we replace $u$ and $v$ by $U$ and $V$ respectively, their sum $(\Phi(x, U, V), \Psi(x, U, V))$ represents a solution of the system (1.1).

Remark. This asymptotic expansion means that, if we write the formal series (II. $2^{\prime}$ ) in the form

(II. $\left.2^{\prime} .1\right) \quad \Phi \approx u+\sum p_{j l}(u) x^{j} v^{l}, \quad \Psi \approx v+\sum q_{j l}(u) x^{j} v^{l}$

with

$$
p_{j l}(u)= \begin{cases}u P_{l}^{(2)}(u) & (j=0), \\ P_{j l}^{(2)}(u) & (j \geqq 1),\end{cases}
$$




$$
q_{j l}(u)= \begin{cases}Q_{l-1}^{\prime 2}(u) & (j=0), \\ Q_{\jmath l}^{\prime 2}(u) & (j \geqq 1),\end{cases}
$$

an inequality of the form

(II. $\left.2^{\prime \prime}\right) \quad\left|\Phi(x, u, v)-u-\sum_{j+l<N} p_{j l}(u) x^{j} v^{l}\right| \leqq K_{N}\left(|x|^{N}+|v|^{N}\right)$

is uniformly satisfied for (29.1), where $N$ is any fixed positive integer and $K_{N}$ is independent of $x, u$ and $v$.

Theorem 5.2.3. There exists a solution $(y, z)=(\Phi(x, U, V)$, $\Psi(x, U, V))$. Here $\Phi(x, u, v)$ and $\Psi(x, u, v)$ are functions holomorphic and bounded in $(x, u, v)$ for (29.1) and uniformly asymptotically developable in the form of (II. 3):

$$
\left\{\begin{array}{l}
\Phi(x, u, v) \approx u\left(1+\sum_{h=0}^{\infty} P_{k}^{(3)}(v) u^{k}\right)+\sum_{j \geqq 1, k \geqq 0} P_{j h}^{(3)}(v) x^{j} u^{k} \\
\Psi(x, u, v) \approx v\left(1+\sum_{h=0}^{\infty} Q_{k}^{(3)}(v) u^{k}\right)+\sum_{\jmath \geqq 1, k \geqq 0} Q_{\jmath_{h}}^{(3)}(v) x^{j} u^{k}
\end{array}\right.
$$

as $x$ and $u$ tend to 0 in the domain (29.1), where the coefficients are the same as those that appeared in Theorem 4.2.3.

In the case when $m=0$, these formal series (II. $3^{\prime}$ ) are uniformly convergent in a domain (29.3) and their sum represents a solution of the system (1.1) if we substitute $U$ and $V$ for $u$ and $v$ respectively.

Remark. The meaning of this asymptotic expansion is similar to that explained in the preceding Remark.

Theorem 5.3. 1. There exists a solution $(y, z)=(\Phi(x, U, V)$, $\Psi(x, U, V))$ such that $\Phi(x, u, v)$ and $\Psi(x, u, v)$ are functions holomorphic and bounded in $(x, u, v)$ for (29.1) and admitting uniformly convergent expansions of the form of (III. 1):

$$
\left\{\begin{array}{l}
\Phi(x, u, v)=u\left(1+P_{0}^{\prime 1}(u, v)\right)+\sum_{j=1}^{\infty} P_{j}^{1^{1}}(u, v) x^{j} \\
\Psi(x, u, v)=v\left(1+Q_{0}^{\prime 1}(u, v)\right)+\sum_{j=1}^{m} Q_{j}^{11}(u, v) x^{j}
\end{array}\right.
$$

Here the coefficients are the same as those appearing in Theorem 4. 3.1 . 
This means that the formal solution (III.1) is uniformly convergent for $(x, U, V)$ in the domain (29.1) and represents a solution of the system (1.1).

Theorem 5.3.2. The system (1.1) possesses a solution $(y, z)$ $=(\Phi(x, U, V), \Psi(x, L, V))$ such that $\Phi(x, u, v)$ and $\Psi(x, u, v)$ are functions holomorphic and bounded in $(x, u, v)$ for (29.1) and, moreover, admitting uniformly asymptotic expansions of the form of (III. 2) :

(III. $2^{\prime}$ )

$$
\left\{\begin{array}{l}
\Phi(x, u, v) \approx \sum_{l=0}^{\infty} P_{l}^{(2)}(x, u) v^{l}, \\
\Psi(x, u, v) \approx \sum_{l=0}^{\infty} Q_{l}^{(2)}(x, u) v^{l},
\end{array}\right.
$$

as $v$ tends to 0 in the domain (29.1), where the coefficients are the same as those that appeared in Theorem 4.3.2.

If $n=0$, the formal series (III. $2^{\prime}$ ) are uniformly convergent for (29.2) and their sum represents a solution of the given system if we substitute $U$ and $V$ for $u$ and $v$ respectively. This means that the formal solution (III.2) is uniformly convergent and represents a true solution for $(x, U, V)$ in the domain (29.2).

Remark. This asymptotic expansion means that, for any positive integer $N$, we have an inequality of the form

$$
\left|\Phi(x, u, v)-\sum_{l=0}^{N-1} P_{i}^{(2)}(x, u) v^{l}\right| \leqq K_{N}|v|^{N}
$$

uniformly in the domain (29.1), $K_{N}$ being a certain positive constant.

Theorem 5. 3. 3. There exists a solution $(y, z)=\Phi((x, U, V)$, $\Psi(x, U, V))$ such that $\Phi(x, u, v)$ and $\Psi(x, u, v)$ are functions holomorphic, bounded in $(x, u, v)$ for (29.1) and uniformly asymptotically developable in the form of (III. 3):

(III. $3^{\prime}$ )

$$
\left\{\begin{array}{l}
\Phi(x, u, v) \approx \sum_{k=0}^{\infty} P_{h}^{(3)}(x, v) u^{k}, \\
\Psi(x, u, v) \approx \sum_{h=0}^{\infty} Q_{h}^{(3)}(x, v) u^{k},
\end{array}\right.
$$


as $u$ tends to 0 in the domain (29.1), where the coefficients are the same functions as in Theorem 4.3.3.

If $m=0$, the formal series (III. $3^{\prime}$ ) is uniformly convergent in a domain (29.3) and their sum represents a true solution of the system (1.1) if we replace $u$ and $v$ by $U$ and $V$ respectively.

Remark. This asymptotic expansion has a meaning analogous to that explained in the preceding Remark.

These theorems will be proved by our usual method for endowing the formal solution with some analytical meaning. According to the very general character of our method, all these theorems are proved in quite a similar way. Hence, the detailed proof will be given for Theorem 5.1 only. For the remaining theorems, we shall only state the outline of the proof to avoid the repetition of similar lengthy reasoning.

Section I. Proof of Theorem 5.1.

30. Peliminary transformation.

Put

$$
\left\{\begin{array}{l}
P_{N}(x, u, v)=u+\sum_{j+k+l<N} P_{j k l} x^{j} u^{k} v^{l}, \\
Q_{N}(x, u, v)=v+\sum_{j+k+l<N} Q_{j k l} x^{j} u^{k} v^{l} .
\end{array}\right.
$$

Since $P_{N}(x, u, v)$ and $Q_{N}(x, u, v)$ are polynomials without constant terms, for any given positive constants $b$ and $b_{1}(<b)$, we have the inequalities of the form

$$
\max \left\{\left|P_{N}(x, u, v)\right|, \quad\left|Q_{N}(x, u, v)\right|\right\}<b-b_{1}
$$

for

$$
|x|<a_{0}, \quad|u|<b_{0}, \quad|v|<b_{0},
$$

if $a_{0}$ and $b_{0}$ are suitably chosen. Moreover, we can suppose without loss of generality that the right-hand members of the differential equations 


$$
x y^{\prime}=f(x, y, z), \quad x z^{\prime}=g(x, y, z)
$$

are functions holomorphic and bounded in $(x, y, z)$ for

$$
|x|<a_{0}, \quad|y|<b_{0}, \quad|z|<b_{0} .
$$

Let us make a transformation

$$
y=\eta+P_{N}(x, U, V), \quad z=\zeta+Q_{N}(x, U, V),
$$

where $(U, V)$ is a holomorphic solution of the reduced system (2.3) or (2.3') (according as we have Case A or Case B) defined in 14. Then the differential equations satisfied by $\eta$ and $\zeta$ will take the form

$$
x \eta^{\prime}=F_{N}(x, U, V, \eta, \zeta), \quad x \zeta^{\prime}=G_{N}(x, U, V, \eta, \zeta),
$$

where

$$
\begin{aligned}
F_{N}(x, u, v, \eta, \zeta)= & f\left(x, \eta+P_{N}(x, u, v), \zeta+Q_{N}(x, u, v)\right) \\
& -x \frac{\partial}{\partial x} P_{N}(x, u, v)-x U^{\prime} \cdot \frac{\partial}{\partial U} P_{N} \\
& -x V^{\prime} \cdot \frac{\partial}{\partial V} P_{N}, \\
G_{N}(x, u, v, \eta, \zeta)= & g\left(x, \eta+P_{N}(x, u, v), \zeta+Q_{N}(x, u, v)\right) \\
& -x \frac{\partial}{\partial x} Q_{N}(x, u, v)-x U^{\prime} \cdot \frac{\partial}{\partial U} Q_{N} \\
& -x V^{\prime} \cdot \frac{\partial}{\partial V} Q_{N} .
\end{aligned}
$$

Here, $x U^{\prime}$ and $x V^{\prime}$ must be replaced by the expressions appearing in the right-hand members of the reduced form (2.3) or (2.3') (according as we are in Case A or Case B).

Hence, we see readily that $F_{N}(x, u, v, \eta, \zeta)$ and $G_{N}(x, u, v, \eta, \zeta)$ are functions holomorphic and bounded in $(x, u, v, \eta, \zeta)$ for

$$
|x|<a_{0}, \quad|u|<b_{0}, \quad|v|<b_{0}, \quad|\eta|<b_{1}, \quad|\zeta|<b_{1} .
$$

It is clear that the differential equations (30.5) have a formal solution of the form

$$
\eta \sim \sum_{j+k+l \geqq N} P_{j k l} x^{j} U^{k} V^{l}, \quad \zeta \sim \sum_{j+k+l \geqq N} Q_{j k l} x^{j} U^{k} V^{l}
$$


with

$$
P_{00 t}=0, \quad Q_{0 k 0}=0 .
$$

Noticing this fact and that the functions $f(0, y, z)$ and $g(0, y, z)$ are of the form

$$
\left\{\begin{array}{l}
f(0, y, z)=y^{m+1} z^{n}\left(\alpha+\sum^{(1)} \alpha_{k l} y^{k} z^{l}\right), \\
g(0, y, z)=y^{m} z^{n+1}\left(\beta+\sum^{(1)} \beta_{k l} y^{k} z^{l}\right),
\end{array}\right.
$$

we can easily verify that inequalities of the form

$$
\begin{aligned}
\left|F_{N}(x, u, v, \eta, \zeta)\right| \leqq & A\left(|x|+|u|^{m}|v|^{n}\right)|\eta| \\
& +A\left(|x|+|u|^{m+1}|v|^{n-1}\right)|\zeta| \\
& +B_{N}\left\{|x|^{N}+|x|\left(|u|^{N-1}+|v|^{N-1}\right)\right. \\
& \left.+|u|^{m+1}|v|^{n}\left(|u|^{N-1}+|v|^{N-1}\right)\right\}
\end{aligned}
$$

$\left(30.9^{\prime}\right) \quad\left|G_{N}(x, u, v, \eta, \zeta)\right| \leqq A\left(|x|+|u|^{m-1}|v|^{n+1}\right)|\eta|$

$$
\begin{aligned}
& +A\left(|x|+|u|^{m}|v|^{n}\right)|\zeta| \\
& +B_{N}\left\{|x|^{N}+|x|\left(|u|^{N-1}+|v|^{N-1}\right)\right. \\
& \left.+|u|^{m}|v|^{n+1}\left(|u|^{N-1}+|v|^{N-1}\right)\right\},
\end{aligned}
$$

(30.10) $\left|F_{N}\left(x, u, v, \eta_{1}, \zeta_{1}\right)-F_{N}\left(x, u, v, \eta_{2}, \zeta_{2}\right)\right|$

$$
\begin{aligned}
& \leqq A\left(|x|+|u|^{m}|v|^{n}\right)\left|\eta_{1}-\eta_{2}\right| \\
& +A\left(|x|+|u|^{m+1}|v|^{n-1}\right)\left|\zeta_{1}-\zeta_{2}\right|,
\end{aligned}
$$

$\left(30.10^{\prime}\right) \quad\left|G_{N}\left(x, u, v, \eta_{1}, \zeta_{1}\right)-G_{N}\left(x, u, v, \eta_{2}, \zeta_{2}\right)\right|$

$$
\begin{aligned}
& \leqq A\left(|x|+|u|^{m-1}|v|^{n+1}\right)\left|\eta_{1}-\eta_{2}\right| \\
& +A\left(|x|+|u|^{m}|v|^{n}\right)\left|\zeta_{1}-\zeta_{2}\right|
\end{aligned}
$$

are satisfied for (30.6), provided that $\eta$ and $\zeta$ satisfy the conditions

$$
|\eta| \leqq C(|x|+|u|), \quad|\zeta| \leqq C(|x|+|v|)
$$

where $C$ is a positive constant. Here $A$ is a suitably chosen positive constant independent of $(x, u, v, \eta, \zeta, N)$, while $B_{N}$ depends on $N$.

Remark. The first two expressions in the right-hand member of (30.9) can be obtained from the estimation of $\left|\frac{\partial}{\partial y} f(x, y, z)\right|$ 
and $\left|\frac{\partial}{\partial z} f(x, y, z)\right|$ for $(y, z)=\left(\eta+P_{N}(x, u, v), \zeta+Q_{N}(x, u, v)\right)$ under the condition (30.11). And, the last one follows from the fact that, in the $\eta$-component of the formal solution (30.7), the terms independent of $x$ contain $U$ as a factor and that $(U, V)$ is a solution of ther educed system (2.3) or $\left(2.3^{\prime}\right)$.

In the case when $n=0$ (or $m=0$ ), we replace the expressions $|v|^{n}$ and $|v|^{n-1}$ (or $|u|^{n}$ and $|u|^{m-1}$ ) by 1 .

The condition (30.11) will be naturally satisfied because of the conditions (30.8).

\section{Auxiliary theorem and proof of Theorem 5.1.}

In order to prove Theorem 5.1, it is sufficient to prove the following auxiliary theorem :

Auxiliary Theorem. The system (30.5) admits one and only one solution $(\eta, \zeta)=\left(\phi_{N}(x, U, V), \phi_{N}(x, U, V)\right)$, where $\phi_{N}(x, u, v)$ and $\psi_{N}(x, u, v)$ are functions holomorphic and bounded in $(x, u, v)$ for

$$
|x|<a_{N}^{\prime \prime}, \quad|u|<b_{N}^{\prime \prime}, \quad|v|<b_{N}^{\prime \prime}, \quad u^{m} v^{n} \in \mathcal{D}\left(c_{N}^{\prime \prime}\right)
$$

and satisfying there the inequalities

(31. 1) $\left|\phi_{N}(x, u, v)\right| \leqq K_{N}\left\{|x|^{N}+(|x|+|u|)\left(|u|^{N-1}+|v|^{N-1}\right)\right\}$,

(31. $\left.1^{\prime}\right) \quad\left|\psi_{N}(x, u, v)\right| \leqq K_{N}\left\{|x|^{N}+(|x|+|v|)\left(|u|^{N-1}+|v|^{N-1}\right)\right\}$.

Here $K_{N}$ is a certain positive constant such that

$$
K_{N}\left(a_{N v}^{\prime N}+2 a_{*}^{\prime \prime} b_{N}^{\prime \prime \prime}+1+2 b_{N}^{\prime \prime}\right)<b_{1} .
$$

Indeed,

$$
\left\{\begin{array}{l}
Y_{N}(x, U, V)=\phi_{N}(x, U, V)+P_{N}(x, U, V), \\
Z_{N}(x, U, V)=\psi_{N}(x, U, V)+Q_{N}(x, U, V)
\end{array}\right.
$$

represent a solution of the original differential equations (1.1). We shall show that $Y_{N}(x, U, V)$ and $Z_{N}(x, U, V)$ are independent of $N$. In fact, for any $N^{\prime}>N$, if $(x, U, V)$ is in the intersection of the domains $(29.1)_{N}$ and $(29.1)_{N^{\prime}}$, 


$$
\left\{\begin{array}{l}
Y_{N^{\prime}}(x, U, V)=\phi_{N^{\prime}}(x, U, V)+P_{N^{\prime}}(x, U, V)-P_{N}(x, U, V), \\
Z_{N^{\prime}}(x, U, V)=\phi_{N^{\prime}}(x, U, V)+Q_{N^{\prime}}(x, U, V)-Q_{N}(x, U, V)
\end{array}\right.
$$

are also the solution of the system (30.5) satisfying the conditions of the Auxiliary Theorem. Hence, such a solution must be equal to $\left(\phi_{N}(x, U, V), \psi_{N}(x, U, V)\right)$. From this it follows that

$$
\left\{\begin{array}{l}
Y_{N^{\prime}}(x, u, v)=Y_{N}(x, u, v)(\equiv \Phi(x, u, v)) \\
Z_{N^{\prime}}(x, u, v)=Z_{N}(x, u, v)(\equiv \Psi(x, u, v))
\end{array}\right.
$$

if $(x, u, v)$ belongs to both $(29.1)_{N}$ and $(29.1)_{N^{\prime}}$. By the analytical continuation, these identities hold for $(x, u, v)$ in the union of these two domains. Therefore, the functions $\Phi(x, u, v)$ and $\Psi(x, u, v)$ are defined in the domain (29.1) with $a_{0}^{\prime \prime}=\sup a_{N}^{\prime \prime}$, $b_{0}^{\prime \prime}=\sup b_{N}^{\prime \prime}, \quad c_{0}^{\prime \prime}=\sup c_{N}^{\prime \prime}$. This proves Theorem 5.1.

So, for the proof of Theorem 5.1, we have only to prove this auxiliary theorem.

Let $\mathfrak{F}$ be the family of the pairs $\left\{\phi_{N}(x, u, v), \psi_{N}(x, u, v)\right\}$ of functions $\varphi_{N}(x, u, v)$ and $\psi_{N}(x, u, v)$, which are holomorphic and bounded in $(x, u, v)$ for

$$
|x|<a_{N}^{\prime \prime}, \quad|u|<b_{N}^{\prime \prime}, \quad|v|<b_{N}^{\prime \prime}, \quad u^{m} v^{n} \in \mathfrak{D}\left(c_{N}^{\prime \prime}\right)
$$

and satisfy there inequalities of the same form as (31.1) and (31. $1^{\prime}$ ) respectively with (31.2).

The domain $\mathfrak{D}(c)$ has been already defined in 14 . Let

$$
U \equiv U\left(x, x_{0}, u^{0}, v^{0}\right), \quad V \equiv V\left(x, x_{0}, u^{0}, v^{0}\right)
$$

be a solution of the system (2.3) or (2.3') holomorphic and satisfying the initial conditions: $U=u^{0}$ and $V=v^{0}$ at $x=x_{0}$, and put

$$
\left\{\begin{array}{l}
\Phi_{N}\left(x_{0}, u^{0}, v^{0}\right)=\int_{0}^{x_{0}} F_{N}\left(x, U, V, \varphi_{N}(x, U, V), \psi_{N}(x, U, V)\right) \frac{d x}{x} \\
\Psi_{N}\left(x_{0}, u^{0}, v^{0}\right)=\int_{0}^{x_{0}} G_{N}\left(x, U, V, \varphi_{N}(x, U, V), \psi_{N}(x, U, V)\right) \frac{d x}{x}
\end{array}\right.
$$

Here $\left(x_{0}, u^{0}, v^{0}\right)$ is an arbitrary point in the domain (29.1) $)_{N}$ and the integration is to be carried out along the curve $\Gamma_{x_{0}}$ which has been already defined in $\mathbf{1 0}$. 
Then, the mapping $\mathfrak{I}$ is defined as follows:

(31.5) $\left\{\varphi_{N}(x, u, v), \psi_{N}(x, u, v)\right\} \stackrel{\Im}{\rightarrow}\left\{\Phi_{N}(x, u, v), \Psi_{N}(x, u, v)\right\}$.

Since $\{0,0\} \in \widetilde{F}, \mathfrak{F}$ is not empty. Moreover, as can be readily verified, the family $\&$ is convex, closed and normal.

On the other hand, owing to Theorem 3 or Theorem $3^{\prime}$ in 9 (according as both of $m$ and $n$ are positive or one of them is zero), the values of the functions $x, U$ and $V$ remain always in the domain $(29.1)_{N}$ when $x$ lies on the path $\Gamma_{x_{0}}$.

Hence, the integrands of the integrals (31.4) are well defined when $x$ is on this path except for $x=0$. Moreover, as we shall show later, the integrals (31.4) converge uniformly. This assures us that the mapping $\mathfrak{I}$ has a well-defined meaning.

Our proof of the Auxiliary Theorem (and Theorem 5.1) is based on the existence of a fixed point of this mapping (see, for example, M. Hukuhara [10]).

\section{Remarks.}

$1^{\circ}$. Here we have supposed that both of $m$ and $n$ are positive integers. However, in the case when one of them is zero, the domain (29.1) $)_{N}$ must be replaced by a domain $(29.2)_{N}$ or (29.3) ${ }_{N}$ similar to (29.2) or (29.3) according as $n=0$ or $m=0$.

$2^{\circ}$. As can be easily seen from the proof, the constants $a_{N}^{\prime \prime}, b_{N}^{\prime \prime}, c_{N}^{\prime \prime}, N$ and $K_{N}$ in the auxiliary theorem must be so chosen that they satisfy certain inequalities (see (34.5), (34.11), (34.10) and $\left.\left(34.10^{\prime}\right)\right)$.

$3^{\circ}$. To complete the proof of Theorem 5.1, it remains for us to prove the inequality $\left(\mathrm{I}^{\prime \prime}\right.$ ) (see the remark following Theorem 5.1). This inequality can be verified as follows:

We take first an integer $N^{\prime}$ satisfying the inequalities remarked above and the inequality $N^{\prime}>N$. Then, by the auxiliary theorem, we have an inequality of the form

$$
\begin{aligned}
\mid \Phi(x, u, v) & -\sum_{\jmath+k+l<N^{\prime}} P_{j k l} x^{j} u^{k} v^{l} \mid \\
& \leqq K_{N^{\prime}}\left(|x|^{N^{\prime}}+(|x|+|u|)\left(|u|^{N^{\prime}-1}+|v|^{N^{\prime}-1}\right)\right) .
\end{aligned}
$$


The last expression is dominated by

$$
\leqq K_{N}\left(|x|^{N-1}+|u|^{N-1}+|v|^{N-1}\right)
$$

provided that

$$
|x|+|u|+|v|<1 \text {. }
$$

Therefore

$$
\begin{aligned}
& \left|\Phi(x, u, v)-\sum_{\jmath+h+l<N} P_{j k l} x^{j} u^{k} v^{l}\right| \\
& \leqq \sum_{N \leqq j_{7} k_{7} l<N}\left|P_{j_{k l} l} x^{j} u^{k} v^{l}\right|+K_{N}\left(|x|^{N^{\prime}-1}+|u|^{N^{\prime}-1}+|v|^{N^{\prime}-1}\right) \\
& \leqq K_{N}\left(|x|^{N}+|u|^{N}+|v|^{N}\right)
\end{aligned}
$$

for a certain positive constant $K_{N}$, if we notice that

$$
\sum_{N \leqq J+\infty, l<N}\left|P_{j k l} x^{j} u^{k} v^{v}\right|=O\left(|x|^{N}+|u|^{N}+|v|^{N}\right) .
$$

This proves the inequality $\left(\mathrm{I}^{\prime \prime}\right)$.

\section{Auxiliary lemmas.}

Before going into the essential part of the proof, two lemmas must be established.

We make the following Assumptions:

i) $\mathcal{D}$ is a simply connected domain in a complex plane such that the origin is an inner point.

ii) $\mathfrak{D}_{r}$ is a simply connected domain in the product space of $r$ complex planes.

iii) $\mathscr{S}_{\mathcal{C}}$ is a simply connected domain in the product space of $r$ complex planes.

iv) $g_{j}\left(x, y_{1}, \cdots, y_{N}\right)(j=1,2, \cdots, L)$ are functions holomorphic and bounded in $\left(x, y_{1}, \cdots, y_{N}\right)$ for

$$
x \in \mathfrak{T}, \quad\left(y_{1}, \cdots, y_{N}\right) \in \mathfrak{D}_{N} .
$$

v) $h_{k}\left(x, u_{1}, \cdots, u_{M}\right)(k=1,2, \cdots, M)$ and $\phi_{i}\left(x, u_{1}, \cdots, u_{M}\right) \quad(i=$ $1,2, \cdots, N)$ are functions holomorphic and bounded in $\left(x, u_{1}, \cdots\right.$, $u_{M}$ ) for

$$
x \in \mathcal{D}, \quad\left(u_{1}, \cdots, u_{M}\right) \in \mathscr{S}_{M} .
$$


vi) $U_{k} \equiv U_{k}\left(x, x_{0}, u_{1}^{0}, \cdots, u_{M}^{0}\right)(k=1,2, \cdots, M)$ is a solution of the differential equations

$$
x \frac{d u_{k}}{d x}=h_{k}\left(x, u_{1}, \cdots, u_{M}\right) \quad(k=1, \cdots, M)
$$

such that $u_{k}^{0}=U_{k}\left(x_{0}, x_{0}, u^{0}\right)(k=1, \cdots, M)$, where $\left(x_{0}, u^{0}\right)$ is an arbitrary point in the domain (D).

vii) The integrals

$$
\begin{aligned}
& \Phi_{j}\left(x_{0}, u_{1}^{0}, \cdots, u_{M}^{0}\right) \equiv \Phi_{j}\left(x_{0}, u^{0}\right) \\
& =\int_{\Gamma_{j x_{0}}} g_{\jmath}\left(x, \phi_{1}(x, U), \cdots, \phi_{N}(x, U)\right) \frac{d x}{x} \\
& (j=1, \cdots, L), \\
& \phi_{i}(x, U) \equiv \phi_{i}\left(x, U_{1}, \cdots, U_{M}\right),
\end{aligned}
$$

converge uniformly. Here $\Gamma_{j x_{0}}$ is a curve in $\mathfrak{D}$ joining the origin with $x_{0}$ such that $\left(x, U_{1}, \cdots, U_{M}\right)$ remains in the domain (D) when $x$ is on this path.

viii) If $x_{0}^{\prime}$ is sufficiently near to $x_{0}$, the relations

$$
\begin{aligned}
\Phi_{j}\left(x_{0}, u^{0}\right) & =\int_{\Gamma_{j x_{0}^{\prime}}} \tilde{g}_{j}(x, U) \frac{d x}{x}+\int_{x_{0}^{\prime}}^{x_{0}} \tilde{g}_{j}(x, U) \frac{d x}{x}, \\
\tilde{g}_{j}(x, U) & \equiv g_{j}\left(x, \phi_{j}(x, U), \cdots, \phi_{N}(x, U)\right),
\end{aligned}
$$

are satisfied. Here the last integration should be taken along the segment joining $x_{0}^{\prime}$ with $x_{0}$.

Lemma 5. 1. 1. $\Phi_{j}(x, U) \equiv \Phi_{j}\left(x, U_{1}, \cdots, U_{M}\right) \quad(j=1, \cdots, L) \quad$ is $\quad$ a solution of the differential equations

$$
x \frac{d z_{j}}{d x}=g_{j}\left(x, \phi_{1}(x, U), \cdots, \phi_{N}(x, U)\right) \quad(j=1, \cdots, L)
$$

such that $\Phi_{j}\left(0, u_{1}^{*}, \cdots, u_{M}^{*}\right)=0$, where

$$
u_{i}^{*}=\lim _{x \rightarrow 0} U_{i}\left(x, x_{0}, u_{1}^{0}, \cdots, u_{M}^{0}\right) .
$$

Lemma 5.1.2. If the integrals (I) converge uniformly with respect to $\left(u_{1}^{0}, \cdots, u_{M}^{0}\right)$ for any fixed $x_{0}$, then $\Phi_{j}\left(x, u_{1}, \cdots, u_{M}\right)$ are functions holomorphic and bounded in $\left(x, u_{1}, \cdots, u_{M}\right)$ in the domain 
(D).

These lemmas were established by M. Iwano [1] when the point $x=0$ is on the boundary of $\mathfrak{D}$. Since the discussion goes essentially the same way for our case, we omit the proof here.

\section{Proof of the Auxiliary Theorem.}

We shall prove the following four propositions.

Proposition 5.1.1. The mapping $\mathfrak{I}$ transforms $\widetilde{\mho}$ into itself.

Proposition 5.1.2. $\mathfrak{I}$ is a continuous mapping of $\widetilde{F}$ with respect to the topology of uniform convergence.

Suppose that these two propositions have been verified. Then, by combining the fact mentioned in $\mathbf{3 1}$, we can conclude, by means of a fixed point theorem, the existence of a fixed point of the mapping $\mathfrak{I}$, namely the pair $\left\{\varphi_{N}(x, u, v), \psi_{N}(x, u, v)\right\}$ of $\mathfrak{E}$ such that

$$
\left\{\Phi_{N}(x, n, v), \psi_{N}(x, u, v)\right\}=\left\{\Phi_{N}(x, u, v), \Psi_{N}(x, u, v)\right\} .
$$

Then we can prove the following proposition:

Proposition 5. 1. 3. The element $\left\{\phi_{N}(x, U, V), \psi_{N}(x, U, V)\right\}$ of $\mathfrak{F}$ corresponding to a fixed point of $\mathfrak{I}$ is a solution of the given differential equations (30.5).

This means that there exists a solution of the system (30.5) in the family $\widetilde{F}$. We shall denote it by $\left\{\phi_{N}(x, U, V), \phi_{N}(x, U, V)\right\}$.

Then we can prove our final proposition.

Proposition 5. 1. 4. The solution $\left\{\phi_{N}(x, U, V), \varphi_{N}^{\prime}(x, U, V)\right\}$ of the differential equations (30.5) such that

$$
\left\{\begin{array}{l}
\phi_{N}(x, U, V)=O\left(|x|^{N}+(|x|+|U|)\left(|U|^{N-1}+|V|^{N-1}\right)\right), \\
\psi_{N}(x, U, V)=O\left(|x|^{N}+(|x|+|V|)\left(|U|^{N-1}+|V|^{N-1}\right)\right)
\end{array}\right.
$$

is unique.

Thus, for the proof of the Auxiliary Theorem, we have only to prove these four propositions.

34. Proof of $\mathbb{P r}_{\text {roposition 5.1.1. }}$

For the proof we must show that 
(a) The integrals (31.4) converge;

(b) We have the following inequalities:

$$
\left\{\begin{aligned}
\left|\Phi_{N}\left(x_{0}, u^{0}, v^{0}\right)\right| \leqq K_{N}\left\{\left|x_{0}\right|^{N}\right. & +\left|x_{0}\right|\left(\left|u^{0}\right|^{N-1}+\left|v^{0}\right|^{N-1}\right) \\
& \left.+\left|u^{0}\right|\left(\left|u^{0}\right|^{N-1}+\left|v^{0}\right|^{N-1}\right)\right\} \\
\left|\Psi_{N}\left(x_{0}, u^{0}, v^{0}\right)\right| \leqq K_{N}\left\{\left|x_{0}\right|^{N}\right. & +\left|x_{0}\right|\left(\left|u^{0}\right|^{N-1}+\left|v^{0}\right|^{N-1}\right) \\
& \left.+\left|v^{0}\right|\left(\left|u^{0}\right|^{N-1}+\left|v^{0}\right|^{N-1}\right)\right\}
\end{aligned}\right.
$$

(c) $\Phi_{N}(x, u, v)$ and $\Psi_{N}(x, u, v)$ are functions holomorphic and bounded in $(x, u, v)$ for $(29.1)_{N}$.

Replacing $|\eta|$ and $|\zeta|$ (in the right-hand members of (30.9) and $\left(30.9^{\prime}\right)$ ) by the expressions of the right-hand members of the inequalities (31.1) and (31. $\left.1^{\prime}\right)$ respectively, we have the inequalities

$$
\left|F_{N}\left(x, u, v, \varphi_{N}(x, u, v), \psi_{N}(x, u, v)\right)\right| \leqq \bar{F}_{N}(x, u, v),
$$

$$
\left|G_{N}\left(x, u, v, \varphi_{N}(x, u, v), \psi_{N}(x, u, v)\right)\right| \leqq \bar{\Psi}_{N}(x, u, v),
$$

where

$$
\begin{aligned}
& \widetilde{\widetilde{Y}}_{N}(x, u, v) \equiv\left\{A\left(2|x|+\left.\left|u_{\mid}^{\mid m}\right| v\right|^{n}+|u|^{n+1}|v|^{n-1}\right) K_{N}+B_{N}\right\}|x|^{N} \\
& +\left\{A\left(2|x|+|u|+|v|+|u|^{m}|v|^{n}+|u|^{m+1}|v|^{n-1}\right) K_{N}\right. \\
& \left.+B_{N}\right\}|x|\left(|u|^{N-1}+|v|^{N-1}\right) \\
& +\left\{2 A K_{N}+B_{N}\right\}|u|^{m+1}|v|^{n}\left(|u|^{N-1}+|v|^{N-1}\right), \\
& \left(34.2^{\prime}\right) \quad \overline{\mathscr{S}}_{N}(x, u, v) \equiv\left\{A\left(2|x|+|u|^{m-1}|v|^{n+1}+|u|^{m}|v|^{n}\right) K_{\mathrm{N}}+B_{N}\right\} \mid x_{\left.\right|^{N}} \\
& +\left\{A\left(2|x|+|u|+|v|+|u|^{m-1}|v|^{n}+|u|^{m}|v|^{n}\right) K_{N}\right. \\
& \left.+B_{N}\right\}|x|\left(|u|^{N-1}+|v|^{N-1}\right) \\
& +\left\{2 A K_{N}+B_{N}\right\}|u|^{m}|v|^{n+1}\left(|u|^{N-1}+|v|^{N-1}\right) \text {. }
\end{aligned}
$$

To prove the proposition, we introduce an auxiliary variably $w$ defined by

$$
w=U^{m} V^{n}
$$

Then, as we have already seen in 11, $x, U$ and $V$ canbe regarded as functions of $w$ which were written as $\bar{x}(w)$, $\dot{U}(w)$ and $\tilde{V}(w)$ respectively. Moreover, we have identities of the form 
(34. 4) $\int_{\Gamma_{x_{0}}}\left\{\begin{array}{l}F_{N} \\ G_{N}\end{array}\right\} \frac{d x}{x}=\int_{\Gamma_{w^{0}}^{*}}\left\{\begin{array}{l}\check{F}_{N} \\ \tilde{G}_{N}\end{array}\right\} \frac{1}{m \alpha+n \beta+\left(m \alpha^{\prime}+n \beta^{\prime}\right) w} \frac{d w}{w^{2}}$

(see (5.2) in Theorem 2.1 in 5), where $\Gamma_{x_{0}}$ and $\Gamma_{w^{0}}^{*}$ are the paths already introduced in $\mathbf{1 0}$ and

$$
\begin{gathered}
\tilde{F}_{N}=F_{N}\left(\tilde{x}, \tilde{U}, \tilde{V}, \tilde{\varphi}_{N}, \tilde{\psi}_{N}\right), \quad \tilde{G}_{N}=G_{N}\left(\tilde{x}, \tilde{U}, \tilde{V}, \tilde{\varphi}_{N}, \tilde{\psi}_{N}\right), \\
\tilde{\varphi}_{N}=\varphi_{N}(\tilde{x}, \tilde{U}, \tilde{V}), \quad \tilde{\psi}_{N}=\psi_{N}(\tilde{x}, \tilde{U}, \tilde{V}) .
\end{gathered}
$$

It is to be noticed that, as $x$ tends to 0 along the path $\Gamma_{x_{0}}$, $w$ tends to 0 algon the path $\Gamma_{w^{0}}^{*}$ (see Theorem 2.3 in 5) and that the part of the path $\Gamma_{w^{0}}^{*}$ which is sufficiently near to the origin consists of the segment denoted by $\Gamma^{\prime \prime}$ (see (10.6), (10.7) and (10.9)).

As we have already seen, we have

$$
\begin{gathered}
\tilde{U}(w)=O(|w| \operatorname{Re} \mathfrak{A}), \quad \tilde{V}(w)=O(|w| \operatorname{Re} \mathfrak{B}), \\
\mathfrak{U}=\frac{\alpha}{m \alpha+n \beta}, \quad \mathfrak{B}=\frac{\beta}{m \alpha+n \beta}
\end{gathered}
$$

on the segment $\Gamma^{\prime \prime}$ (see Theorem 2.1 in 5) and the function $\hat{x}(w)$ tends exponentially to 0 along the segment $\Gamma^{\prime \prime}$ (see Theorem 2.3 in 5 and also 8 and 10). Hence, if $N$ satisfies the inequalities

$$
\left\{\begin{array}{l}
N \operatorname{Re} \mathfrak{A} \geqq 1, \quad \operatorname{Re} \mathfrak{U}+(N-1) \operatorname{Re} \mathfrak{B} \geqq 1, \\
(N-1) \operatorname{Re} \mathfrak{A}+\operatorname{Re} \mathfrak{B} \geqq 1, \quad N \operatorname{Re} \mathfrak{B} \geqq 1,
\end{array}\right.
$$

we see by (34.2) and (34. $\left.2^{\prime}\right)$ that the integrals

$$
\int_{\Gamma_{w^{0}}^{*}} \overline{\widetilde{F}}_{N}(\bar{x}, \tilde{U}, \tilde{V}) \frac{d w}{w^{2}} \quad \text { and } \quad \int_{\Gamma_{w 0}^{*}} \overline{\mathcal{S}}_{N}(\hat{x}, \tilde{U}, \tilde{V}) \frac{d w}{w^{2}}
$$

are convergent, which, by (34.1) and (34.1'), proves the convergence of the integrals (31.4).

Thus we have proved (a).

To show that (b) holds, it is sufficient, by (34.1), (34.1') and (34.4), to prove that: 
(34.6)

$$
\begin{aligned}
& \frac{2}{|m \alpha+n \beta|} \int_{\Gamma_{w 0}^{*}} \widetilde{\widetilde{\Im}}_{N}(\tilde{x}, \tilde{U}, \tilde{V})\left|\frac{d w}{w^{2}}\right| \\
& \leqq K_{N}\left\{\left|x_{0}\right|^{N}+\left(\left|x_{0}\right|+\left|u^{0}\right|\right)\left(\left|u^{0}\right|^{N-1}+\left|v^{0}\right|^{N-1}\right)\right\}
\end{aligned}
$$

$\left(34.6^{\prime}\right) \frac{2}{|m \alpha+n \beta|} \int_{\Gamma_{w 0}^{*}} \overline{\mathcal{B}}_{N}(\tilde{x}, \tilde{U}, \tilde{V})\left|\frac{d w}{w^{2}}\right|$

$$
\leqq K_{N}\left\{\left|x_{0}\right|^{N}+\left(\left|x_{0}\right|+\left|v^{0}\right|\right)\left(\left|u^{0}\right|^{N-1}+\left|v^{0}\right|^{N-1}\right)\right\},
$$

if we notice that, for sufficiently small $|w|$,

$$
\left|m \alpha+n \beta+\left(m \alpha^{\prime}+n \beta^{\prime}\right) w\right| \geqq \frac{1}{2}|m \alpha+n \beta| .
$$

Let $s$ be the length of the arc $\Gamma_{w^{0}}^{*}$ measured from the origin to an arbitrary point $w$ on $\Gamma_{w 0}^{*}$. Then, as we have already shown, we have

$$
\left|\frac{d w}{d s}\right|=1
$$

(See (12.5) and (12.12) in 12). Hence, the inequalities (34.6) and $\left(34.6^{\prime}\right)$ are equivalent to

$$
\frac{2}{|m \alpha+n \beta|} \int_{0}^{s} \overline{\widetilde{r}}_{N} \frac{d s}{|w|^{2}} \leqq K_{N}\left\{|x|^{N}+(|x|+|\tilde{U}|)\left(|\tilde{U}|^{N-1}+|\tilde{V}|^{N-1}\right)\right\}
$$

and

(34. 8') $\frac{2}{|m \alpha+n \beta|} \int_{0}^{s} \overline{\mathbb{S}}_{N} \frac{d s}{|w|^{2}} \leqq K_{N}\left\{|\hat{x}|^{N}+(|\bar{x}|+|\tilde{V}|)\left(|\tilde{U}|^{N-1}+|\tilde{V}|^{N-1}\right)\right\}$.

respectively. These inequalities can be derived from the inequalities

$$
\begin{aligned}
\frac{2 \overline{\widetilde{F}}_{N}(\tilde{x}, \tilde{U}, \tilde{V})}{|m \alpha+n \beta||U|^{2 m}|V|^{2 n}} & \leqq K_{N} \frac{d}{d s}\left\{|\bar{x}|^{N}\right. \\
& \left.+(|\bar{x}|+|\tilde{U}|)\left(|\tilde{U}|^{N-1}+|\tilde{V}|^{N-1}\right)\right\}
\end{aligned}
$$

and

$$
\begin{aligned}
\frac{2 \overline{\mathscr{S}}_{N}(\tilde{x}, \tilde{U}, \tilde{V})}{|m \alpha+n \beta||U|^{2 m}|V|^{2 n}} & \leqq K_{N} \frac{d}{d s}\left\{|\tilde{x}|^{N}\right. \\
& \left.+(|x|+|\tilde{V}|)\left(|\tilde{U}|^{N-1}+|\tilde{V}|^{N-1}\right)\right\}
\end{aligned}
$$


on the curve $\Gamma_{w 0}^{*}$, since $w=\tilde{U}^{m} \tilde{V}^{n}$.

By virtue of Lemma 3.2 in 11 and since $\rho=|w|=|\tilde{U}|^{m}|\tilde{V}|^{n}$, we have

$$
\begin{aligned}
\frac{d}{d s}\left\{|\tilde{x}|^{N}\right. & \left.+(|\tilde{x}|+|\tilde{U}|)\left(|\tilde{U}|^{N-1}+|\tilde{V}|^{N-1}\right)\right\} \\
& \geqq \frac{\sin \delta_{0}}{2|m \alpha+n \beta||\tilde{U}|^{m m}|\tilde{V}|^{2 n}}\left[N|\tilde{x}|^{N}+|\tilde{x}|\left(|\tilde{U}|^{N-1}+|\tilde{V}|^{N-1}\right)\right. \\
& +(N-1)|\tilde{U}|^{m}|\tilde{V}|^{n}|\tilde{x}|\left(|\alpha||\tilde{U}|^{N-1}+|\beta||\tilde{V}|^{N-1}\right) \\
& \left.+|\tilde{U}|^{m+1}|\tilde{V}|^{n}\left(N|\alpha||\tilde{U}|^{N-1}+(|\alpha|+(N-1)|\beta|)|\tilde{V}|^{N-1}\right)\right] \\
& \geqq \frac{\sin \delta_{0}}{2|m \alpha+n \beta||\tilde{U}|^{2 m}|\tilde{V}|^{2 n}}\left[N|\bar{x}|^{N}+\{1+(N-1)\right. \\
& \left.\times \min (|\alpha|,|\beta|)|\tilde{U}|^{m}|\tilde{V}|^{n}\right\}|\tilde{x}|\left(|\tilde{U}|^{N-1}+|\tilde{V}|^{N-1}\right) \\
& \left.+N \min (|\alpha|,|\beta|)|\tilde{U}|^{m+1}|\tilde{V}|^{n}\left(|\tilde{U}|^{N-1}+|\tilde{V}|^{N-1}\right)\right]
\end{aligned}
$$

and similarly,

$$
\begin{aligned}
\frac{d}{d s}\left\{|\ddot{x}|^{N}\right. & \left.+(|\bar{x}|+|\tilde{V}|)\left(|\hat{U}|^{N-1}+|\tilde{V}|^{N-1}\right)\right\} \\
& \geqq \frac{\sin \delta_{0}}{2|m \alpha+n \beta||U|^{2 m}|\tilde{V}|^{2 n}}\left[N|\tilde{x}|^{N}+\{1+(N-1)\right. \\
& \left.\times \min (|\alpha|,|\beta|)|\tilde{U}|^{m}|\tilde{V}|^{n}\right\}|\tilde{x}|\left(|\tilde{U}|^{N-1}+|\tilde{V}|^{N-1}\right) \\
& \left.+N \min (|\alpha|,|\beta|)|\tilde{U}|^{m}|\hat{V}|^{n+1}\left(|\tilde{U}|^{N-1}+|\tilde{V}|^{N-1}\right)\right] .
\end{aligned}
$$

Therefore, since $\overline{\mathfrak{F}}_{N}(x, u, v)$ and $\overline{\mathbb{S}}(x, u, v)$ are defined by (34.2) and $\left(34.2^{\prime}\right)$ respectively, we see that (34.9) and (34. $\left.9^{\prime}\right)$ are an immediate consequence of the inequalities

$$
\left\{\begin{array}{c}
4 A\left(2|\tilde{x}|+|\tilde{U}|^{m}|\tilde{V}|^{n}+|\tilde{U}|^{m+1}|\hat{V}|^{n-1}\right) K_{N}+4 B_{N}<N \sin \delta_{0} \cdot K_{N} \\
4 A\left(2|\tilde{x}|+|\tilde{U}|+|\bar{V}|+|\tilde{U}|^{m}|\tilde{V}|^{n}+|\hat{U}|^{m+1}|\tilde{V}|^{n-1}\right) K_{N} \\
+4 B_{N}<\sin \delta_{0} \cdot K_{N} \\
8 A K_{N}+4 B_{N}<N \min (|\alpha|,|\beta|) \sin \delta_{0} \cdot K_{N}
\end{array}\right.
$$

and

$$
\left\{\begin{array}{c}
4 A\left(2|\tilde{x}|+|\check{U}|^{m-1}|\tilde{V}|^{n+1}+|\tilde{U}|^{m}|\tilde{V}|^{n}\right) K_{N}+4 B_{N}<N \sin \delta_{0} \cdot K_{N} \\
4 A\left(2|\tilde{x}|+|\tilde{U}|+|\tilde{V}|+|\tilde{U}|^{m-1}|\tilde{V}|^{n+1}+|\tilde{U}|^{m}|\tilde{V}|^{n}\right) K_{N} \\
\quad+4 B_{N}<\sin \delta_{0} \cdot K_{N} \\
8 A K_{N}+4 B_{N}<N \min (|\alpha|,|\beta|) \sin \delta_{0} \cdot K_{N}
\end{array}\right.
$$


respectively.

We take first the quantities $N, 1 / a_{N}^{\prime \prime}$ and $1 / b_{N}^{\prime \prime}$ so large that the following two inequalities are satisfied:

$$
\left\{\begin{array}{l}
8\left(a_{N}^{\prime \prime}+b_{N}^{\prime \prime}+\left(b_{N}^{\prime \prime}\right)^{m+n}\right) A<\sin \delta_{0}, \\
8 A<N \min (|\alpha|,|\beta|) \sin \delta_{0} .
\end{array}\right.
$$

and next $K_{N}$ sufficiently large so that the inequalities (34.10) and $\left(34.10^{\prime}\right)$ are satisfied for the values of $\bar{x}(w), \tilde{U}(w), \tilde{V}(w)$ in the domain $(29.1)_{N}$. This is evidently possible.

Thus we have proved (b).

In order to prove (c), we consider the following correspondence :

(1) The reduced system (2.3) or (2. $\left.3^{\prime}\right)$ to the equations (E) in 32 ;

(2) The integrands of (31.4) to the integrands of I in 32 ;

(3) The pair $\left\{\Phi_{N}(x, u, v), \Psi_{N}(x, u, v)\right\}$ of the functions $\Phi_{N}(x, u, v)$ and $\Psi_{N}(x, u, v)$ defined by (31.4) to the system $\left\{\Phi_{1}\left(x, u_{1}, \cdots, u_{M}\right), \cdots, \Phi_{L}\left(x, u_{1}, \cdots, u_{M}\right)\right\}$ of the functions $\Phi_{j}\left(x, u_{1}, \cdots\right.$, $u_{M}$ ) defined by the integrals (I);

(4) The path $\Gamma_{x_{0}}$ in (31.4) to the path $\Gamma_{j x_{0}}$ in (I).

Then the relation ( $R$ ) in 32 becomes

$$
\int_{\Gamma_{x_{0}}} F_{N}\left(x, U, V, \mathcal{P}_{N}, \psi_{N}\right) \frac{d x}{x}=\int_{\Gamma_{x_{0}^{\prime}}} F_{N} \frac{d x}{x}+\int_{x_{0}^{\prime}}^{r_{0}} F_{N} \frac{d x}{x}
$$

and

(34.12') $\int_{\Gamma^{\prime} x_{0}} G_{N}\left(x, U, V, \varphi_{N}, \psi_{N}\right) \frac{d x}{x}=\int_{\Gamma_{x_{0}^{\prime}}} G_{N} \frac{d x}{x}+\int_{x_{0}^{\prime}}^{x_{0}} G_{N} \frac{d x}{x}$.

We suppose that these relations are verified. Then, since the assertion (b) just established assures us the uniform convergence of the integrals (31.4), we see that (c) is an immediate consequence of Lemma 5.1.2 in 32 .

Therefore, to obtain (c), it is sufficient to prove that the relations (34.12) and (34.12') are satisfied. 
Let $\xi(\rho)$ and $\xi^{\prime}(\rho)$ be the points of intersection of a sufficiently small circle $|x|=\rho$ with the paths $\Gamma_{x_{0}}$ and $\Gamma x_{0}^{\prime}$ respectively. Then, to obtain (34.12) and (34.12') it is sufficient to show that

$$
\left|\int_{\xi(\rho)}^{\xi^{\prime}(\rho)} F_{N} \frac{d x}{x}\right| \rightarrow 0, \quad\left|\int_{\xi(\rho)}^{\xi^{\prime}(\rho)} G_{N} \frac{d x}{x}\right| \rightarrow 0
$$

as $\rho \rightarrow 0$, where the integration should be taken along the arc of the circle $|x|=\rho$.

To prove (34.13), let us introduce again an auxiliary variable $w$ by the relation (34.3). Then, (34.13) will be reduced to

$$
\begin{gathered}
\left|\int_{w_{1}}^{w_{1}^{\prime}} \frac{\tilde{F}_{N}}{X(w)} d w\right| \rightarrow 0, \quad\left|\int_{w_{1}}^{w_{1}^{\prime}} \frac{\tilde{G}_{N}}{X(w)} d w\right| \rightarrow 0, \\
X(w)=\left(m \alpha+n \beta+\left(m \alpha^{\prime}+n \beta^{\prime}\right) w\right) w^{2},
\end{gathered}
$$

where $\tilde{F}_{N}$ and $\tilde{G}_{N}$ are the same functions as those appearing in (34.4), and the integration should be taken along a curve $L\left(w_{1}, w_{1}^{\prime}\right)$ which will be defined as follows:

Let

$$
w^{0}=\left(u^{0}\right)^{m}\left(v^{0}\right)^{n}, \quad w^{0}=\left(u^{0}\right)^{m}\left(v^{0}\right)^{n},
$$

where $\left(u^{0}, v^{0}\right)$ and $\left(u^{0}, v^{0}\right)$ denote the values of $(U, V)$ at $x=x_{0}$ and $x=x_{0}^{\prime}$ respectively, and we determine the curves $\Gamma_{w^{0}}^{*}$ and $\Gamma_{w^{0^{\prime}}}^{*}$ in the same way as in 10. The paths $\Gamma_{x_{0}}$ and $\Gamma_{x_{0}^{\prime}}$ are respectively the images of the curves $\Gamma_{w 0}^{*}$ and $\Gamma_{w^{\prime}}^{*}$ by the transformation defined by the first relation of (5.3) (where $C_{1}$ must be so chosen that this relation holds for $x=x_{0}$ and $\left.w=w^{0}\right)$. Then, the curve $L\left(w_{1}, w_{1}^{\prime}\right)$ must be so chosen that an image of this curve by the same transformation becomes the arc $\widehat{\xi(\rho) \xi^{\prime}(\rho)}$.

If $\rho$ is sufficiently small, the point of the curve $L\left(w_{1}, w_{1}^{\prime}\right)$ is located in the sector

$$
\theta_{-}+2 \delta_{0} \leqq \arg w \leqq \theta_{+}-2 \delta_{0},
$$

in which the function $\tilde{x}(w)$ tends exponentially to 0 and the functions $\tilde{U}(w)$ and $\check{V}(w)$ satisfy the following condition (see 
Theorem 2.1 in 5): $\tilde{U}(w)=O(|w| \operatorname{Re} \mathfrak{A}), \quad \tilde{V}(w)=O(|w| \operatorname{Re} \mathfrak{B})$.

Since the maximum of $|w|$ for $w \epsilon L\left(w_{1}, w_{1}^{\prime}\right)$ approaches 0 with $\rho$, we can verify, as in the proof of (a), that the expressions in the left-hand members of (34.14) and, consequently, those of (34.13) converge to 0 with $\rho$.

Thus Proposition 5.1.1 has been completely proved.

\section{Proof of Propositions 5. 1.2, 5.1.3 and 5.1.4.}

$1^{\circ}$. To prove Proposition 5.1.2, it is sufficient to prove that if the sequence $\left\{\varphi_{N, l}(x, u, v), \psi_{N, l}(x, u, v)\right\}$ tends to 0 with respect to the topology of $\mathfrak{F},\left\{\Phi_{N, l}(x, u, v), \Psi_{N, l}(x, u, v)\right\}$ also tends to 0 .

Since the topology of $₹$ is defined by uniform convergence, this assertion is almost evident from the proof of the assertion (b) of Proposition 5.1.1.

$2^{\circ}$. Proposition 5.1 .3 is an immediate consequence of the assertion that, for any $\left\{\varphi_{N}(x, u, v), \psi_{N}(x, u, v)\right\} \in \mathfrak{F},\left\{\Phi_{N}(x, U, V)\right.$, $\left.\Psi_{N}(x, U, V)\right\}$ is a solution of the differential equations

$$
\left\{\begin{array}{l}
x \eta^{\prime}=F_{N}\left(x, U, V, \varphi_{N}(x, U, V), \psi_{N}(x, U, V)\right), \\
x \xi^{\prime}=G_{N}\left(x, U, V, \varphi_{N}(x, U, V), \psi_{N}(x, U, V)\right) .
\end{array}\right.
$$

However, this assertion can be readily derived from Lemma 5. 1.1 in 32 .

$3^{\circ}$. Suppose that there exist two solutions satisfying the same conditions. Let $\left\{\phi^{*}(x, U, V), \psi^{*}(x, U, V)\right\}$ be the difference of such two solutions. Then, by virtue of the inequalities (30.10) and (30.10'), we have

$$
\begin{aligned}
& \text { (35.1) } \begin{aligned}
\left|\varphi^{*}(x, U, V)\right| \leqq & A \mid \int_{0}^{x}\left\{\left(|x|+|U|^{m}|V|^{n}\right)\left|\varphi^{*}(x, U, V)\right|\right. \\
& \left.+\left(|x|+|U|^{m+1}|V|^{n-1}\right)\left|\psi^{*}(x, U, V)\right|\right\} \frac{d x}{x} \mid, \\
\left(35.1^{\prime}\right)\left|\psi^{*}(x, U, V)\right| \leqq & A \mid \int_{0}^{x}\left\{\left(|x|+|U|^{m-1}|V|^{n+1}\right)\left|\varphi^{*}(x, U, V)\right|\right. \\
& \left.+\left(|x|+|U|^{m}|V|^{n}\right)\left|\psi^{*}(x, U, V)\right|\right\} \frac{d x}{x} \mid
\end{aligned}
\end{aligned}
$$


Put

$$
\begin{array}{r}
K^{*}=\max \left\{\sup _{(x, u, v)}\left|\varphi^{*}(x, u, v)\right|\left(|x|^{N}+(|x|+|u|)\left(|u|^{N-1}+|v|^{N-1}\right)\right)^{-1},\right. \\
\left.\sup _{(x, u, v)}\left|\psi^{*}(x, u, v)\right|\left(|x|^{N}+(|x|+|v|)\left(|u|^{N-1}+|v|^{N-1}\right)\right)^{-1}\right\}
\end{array}
$$

where the point $(x, u, v)$ should run over the domain $(29.1)_{N}$.

To obtain Proposition 5.1.4, it is sufficient to prove that $K^{*}=0$.

Suppose that $0<K^{*}<+\infty$. The definition of $K^{*}$ implies that:

$\left|\phi^{*}(x, U, V)\right| \leqq K^{*}\left\{|x|^{N}+(|x|+|U|)\left(|U|^{N-1}+|V|^{N-1}\right)\right\}$,

Now we substitute the expressions appearing in the righthand members of (35.2) and $\left(35.2^{\prime}\right)$ for $\varphi^{*}$ and $\psi^{*}$ in the righthand members of $(35.1)$ and $\left(35.1^{\prime}\right)$. Then, the resulting inequalities can be written as

$$
\left\{\begin{array}{l}
\left|\varphi^{*}(x, U, V)\right| \leqq \mid \int_{0}^{x} \tilde{\widetilde{F}}_{N}(x, U, V) \frac{d x}{x}, \\
\left|\psi^{*}(x, U, V)\right| \leqq \mid \int_{0}^{x} \tilde{\mathscr{S}}_{N}(x, U, V) \frac{d x}{x} .
\end{array}\right.
$$

Here, $\tilde{\mathfrak{F}}_{N}(x, u, v)$ and $\tilde{\mathbb{S}}_{N}(x, u, v)$ are given by the formulae (34.2) and (34. $\left.2^{\prime}\right)$ respectively, where we must put $K_{N}=K^{*}$ and $B_{N}=0$. Observe that, in the inequalities (34.10) and (34.10'), the equality sign can never be realized. Hence, we can obtain, by the same calculation as in the proof of the assertion (b) of Proposition 5.1.1 in 34, the inequalities

$$
\left\{\begin{array}{l}
\left|\phi^{*}(x, U, V)\right|<K^{*}\left\{|x|^{N}+(|x|+|U|)\left(|U|^{N-1}+|V|^{N-1}\right)\right\}, \\
\left|\psi^{*}(x, U, V)\right|<K^{*}\left\{|x|^{N}+(|x|+|V|)\left(|U|^{N-1}+|V|^{N-1}\right)\right\} .
\end{array}\right.
$$

From this it follows that $K^{*}<K^{*}$. Hence, we have $0<K^{*}<+\infty$, which is the contradiction. Therefore, $K^{*}$ must be equal to zero.

Thus we have proved completely Theorem 5.1. 
Section II. Proof of Theorem 5.2.1.

\section{Outline of the proof.}

We put

$$
\left\{\begin{array}{l}
P_{N}(x, u, v)=u+\sum_{k+l<N} P_{k l}^{(1)}(x) u^{k} v^{l} \\
Q_{N}(x, u, v)=v+\sum_{k+l<N} Q_{k i}^{(1)}(x) u^{k} v^{l}
\end{array}\right.
$$

and make a transformation of the form

$$
y=\eta+P_{N}(x, U, V), \quad z=\zeta+Q_{N}(x, U, V) .
$$

Let

$$
x \eta^{\prime}=F_{N}(x, U, V, \eta, \zeta), \quad x \zeta^{\prime}=G_{N}(x, U, V, \eta, \zeta)
$$

be the system derived from the system (1.1) by the transformation (36.2). Clearly, this system admits a formal solution of the form

$$
\eta \sim \sum_{k+l \geqq N} P_{h l}^{(1)}(x) U^{k} V^{l}, \quad \zeta \sim \sum_{h+l \geqq N} Q_{k l}^{(1)}(x) U^{k} V^{l}
$$

with

$$
P_{0 l}^{(1)}(0)=0, \quad Q_{k 0}^{(1)}(0)=0 \quad(k, l=N, N+1, \cdots) .
$$

Moreover, we can easily verify that:

1) $F_{N}(x, u, v, \eta, \zeta)$ and $G_{N}(x, u, v, \eta, \zeta)$ are functions holomorphic and bounded in $(x, u, v, \eta, \zeta)$ for

$$
|x|<a_{0},|u|<b_{0}, \quad|v|<b_{0}, \quad|\eta|<b_{1}, \quad|\zeta|<b_{1},
$$

where $a_{0}, b_{0}$ and $b_{1}$ are suitably chosen positive constants;

2) $F_{N}(x, u, v, \eta, \zeta)$ and $G_{N}(x, u, v, \eta, \zeta)$ satisfy there the inequalities

(36. 7) $\left|F_{N}\right| \leqq A\left(|x|+|u|^{m}|v|^{n}\right)|\eta|+A\left(|x|+|u|^{m+1}|v|^{n-1}\right)|\zeta|$

$$
+B_{N}\left\{|x|\left(|u|^{N}+|v|^{N}\right)+|u|^{m+1}|v|^{n}\left(|u|^{N-1}+|v|^{N-1}\right)\right\}
$$


(36. $\left.7^{\prime}\right) \quad\left|G_{N}\right| \leqq A\left(|x|+|u|^{m-1}|v|^{n+1}\right)|\eta|+A\left(|x|+|u|^{m}|v|^{n}\right)|\zeta|$

$$
+B_{N}\left\{|x|\left(|u|^{N}+|v|^{N}\right)+|u|^{m}|v|^{n+1}\left(|u|^{N-1}+|v|^{N-1}\right)\right\}
$$

and, moreover, we have inequalities of the forms (30.10) and $\left(30.10^{\prime}\right)$ provided that $\eta$ and $\zeta$ satisfy the conditions

$$
|\eta| \leqq C(|x|+|u|), \quad|\zeta| \leqq C(|x|+|v|),
$$

where $C$ is a positive constant.

Remark. The last term appearing in the expression in the right-hand member of (36.7) or (36. $\left.7^{\prime}\right)$ can be obtained from the fact that the differential equations (36.3) possess a formal solution of the form (36.4) with the initial values (36.5) and $(U, V)$ is a solution of the reduced system (2.3) or $\left(2.3^{\prime}\right)$.

\section{Auxiliary theorem.}

To obtain Theorem 5.2.1, it is sufficient to prove the following auxiliary theorem:

Auxiliary Theorem. The differential equations (36.3) admit a solution $(\eta, \zeta)=\left(\phi_{N}(x, U, V), \phi_{N}(x, U, V)\right)$, where $\phi_{N}(x, u, v)$ and $\phi_{N}(x, u, v)$ are functions holomorphic and bounded in $(x, u, v)$ for

$$
|x|<a_{N}^{\prime \prime},|u|<b_{N}^{\prime},|v|<b_{N}^{\prime \prime}, u^{m} v^{n} \in \mathfrak{D}\left(c_{N}^{\prime \prime}\right)
$$

and satisfying there the inequalities

$$
\left\{\begin{array}{l}
\left|\phi_{N}\right| \leqq K_{N}\left\{|x|\left(|u|^{N-1}+|v|^{N-1}\right)+|u|\left(|u|^{N-1}+|v|^{N-1}\right)\right\} \\
\left|\phi_{N}\right| \leqq K_{N}\left\{|x|\left(|u|^{N-1}+|v|^{N-1}\right)+|v|\left(|u|^{N-1}+|v|^{N-1}\right)\right\}
\end{array}\right.
$$

with

$$
2\left(a_{\Lambda}^{\prime \prime} b_{N}^{\prime \prime}-1+b_{N}^{\prime \prime N}\right) K_{N}<b_{1} .
$$

Moreover, the solution of (36.3) satisfying the conditions (37. 1) is unique.

Indeed, the functions

$$
\left\{\begin{array}{l}
Y_{N}(x, U, V)=P_{N}(x, U, V)+\phi_{N}(x, U, V), \\
Z_{N}(x, U, V)=Q_{N}(x, U, V)+\psi_{N}(x, U, V)
\end{array},\right.
$$

represent a solution of the system (1.1). And, by the same 
reasoning as in 31 , we see that these functions are independent of $N$ if $N$ is chosen so large as to satisfy the inequalities (34.5).

Thus Theorem 5.2.1 has been proved.

The auxiliary theorem can be established without any essential modification of the reasoning given in 31-35. As regards the family $\mathfrak{F}$, for example, it is sufficient to take the pairs $\left\{\varphi_{N}(x, u, v), \psi_{N}(x, u, v)\right\}$ of functions $\varphi_{N}(x, u, v)$ and $\psi_{N}(x, u, v)$ such that they are holomorphic and bounded in $(x, u, v)$ for

$$
|x|<a_{N}^{\prime \prime}, \quad|u|<b_{N}^{\prime \prime}, \quad|v|<b_{N}^{\prime \prime}, \quad u^{m} v^{n} \in \mathfrak{D}\left(c_{N}^{\prime \prime}\right)
$$

and satisfy there the inequalities

$$
\text { (37. 1') }\left\{\begin{array}{l}
\left|\varphi_{N}\right| \leqq K_{N}\left\{|x|\left(|u|^{N-1}+|v|^{N-1}\right)+|u|\left(|u|^{N-1}+|v|^{N-1}\right)\right\} \\
\left|\psi_{N}\right| \leqq K_{N}\left\{|x|\left(|u|^{N-1}+|v|^{N-1}\right)+|v|\left(|u|^{N-1}+|v|^{N-1}\right)\right\}
\end{array}\right.
$$

with (37.2).

Remark. From the form of the formal solution (36.4), it seems to me that the inequalities (37.1) may be replaced by

$$
\left\{\begin{array}{l}
\left|\phi_{N}\right| \leqq K_{N}\left\{|x|\left(|u|^{N}+|v|^{N}\right)+|u|\left(|u|^{N-1}+|v|^{N-1}\right)\right\} \\
\left|\psi_{N}\right| \leqq K_{N}\left\{|x|\left(|u|^{N}+|v|^{N}\right)+|v|\left(|u|^{N-1}+|v|^{N-1}\right)\right\}
\end{array}\right.
$$

although I could not prove the existence of such a solution. However, to obtain Theorem 5.2.1, it is sufficient to prove the auxiliary theorem.

Section III. Proof of Theorems 5.2.2 and 5.2.3.

\section{Outline of the proof.}

Since the proof of Theorem 5.2.3 is essentially similar to that of Theorem 5.2.2, we shall expalin the outline of the proof of Theorem 5.2.2 for the case $n>0$ only.

As we have already remarked in the Remark following Theorem 5.2.2, we must rewrite the formal solution (II. 2) in the form (II. 2'.1) in 29 (where $u$ and $v$ must be replaced by $U$ and $V$ ): 


$$
y \sim U+\sum p_{j l}(U) x^{j} V^{\imath}, \quad z \sim V+\sum q_{j l}(U) x^{j} V^{\imath} .
$$

Theorem 4.2.2 in 14 shows that the functions $p_{0 r}(u)$ and $q_{02}(u)$ are holomorphic at $u=0$ and, especially,

$$
q_{00}(u) \equiv 0, \quad p_{0 z}(0)=0 \quad(l=0,1, \cdots) .
$$

This can be easily verified from the fact that, in the $y$-component of the formal solution, the terms independent of $x$ contain $U$ as a factor.

Let us put

$$
\left\{\begin{array}{l}
P_{N}(x, u, v)=u+\sum_{j+l<N} p_{j l}(u) x^{j} v^{l} \\
Q_{N}(x, u, v)=v+\sum_{j+l<N} q_{j l}(u) x^{j} v^{l},
\end{array}\right.
$$

and make a transformation of the form

$$
y=\eta+P_{N}(x, U, V), \quad z=\zeta+Q_{N}(x, U, V) .
$$

The differential equations (1.1) will be transformed into

$$
x \eta^{\prime}=F_{N}(x, U, V, \eta, \zeta), \quad x \zeta^{\prime}=G_{N}(x, U, V, \eta, \zeta)
$$

which admit a formal solution of the form

$$
\eta \sim \sum_{j+l \geqq N} p_{j l}(U) x^{j} V^{\imath}, \quad \zeta \sim \sum_{j+l \geqq N} q_{j l}(U) x^{j} V^{\imath}
$$

with the condition (38.1).

Keeping this fact in mind, we can easily prove that:

1) $F_{N}(x, u, v, \eta, \zeta)$ and $G_{N}(x, u, v, \eta, \zeta)$ are functions holomorphic and bounded in $(x, u, v, \eta, \zeta)$ for

$$
|x|<a_{0}, \quad|u|<b_{0}, \quad|v|<b_{0}, \quad|\eta|<b_{1}, \quad|\zeta|<b_{1} ;
$$

2) $F_{N}(x, u, v, \eta, \zeta)$ and $G_{N}(x, u, v, \eta, \zeta)$ satisfy there the inequalities

$$
\begin{aligned}
\left|F_{N}\right| & \leqq A\left(|x|+|u|^{m}|v|^{n}\right)|\eta|+A\left(|x|+|u|^{m+1}|v|^{n-1}\right)|\zeta| \\
& +B_{N}\left(|x|^{N}+|x||v|^{N-1}+|u|^{m}|v|^{n} \cdot|u||v|^{N-1}\right)
\end{aligned}
$$

$$
\begin{aligned}
\left|G_{N}\right| & \leqq A\left(|x|+|u|^{m-1}|v|^{n+1}\right)|\eta|+A\left(|x|+|u|^{m}|v|^{n}\right)|\zeta| \\
& +B_{N}\left(|x|^{N}+|x||v|^{N-1}+|u|^{m}|v|^{n} \cdot|v|^{N}\right)
\end{aligned}
$$


and, moreover, we have the inequalities (30.10) and (30.10') provided that the conditions

$$
|\eta| \leqq C(|x|+|u|), \quad|\zeta| \leqq C(|x|+|v|)
$$

are satisfied, where $C$ is a positive constant.

To obtain Theorem 5.2.2, it is sufficient to prove the following auxiliary theorem :

Auxiliary Theorem. The differential equations (38.4) admit one and only one solution $\left(\phi_{N}(x, U, V), \psi_{N}{ }_{N}(x, U, V)\right)$ such that $\phi_{N}(x, u, v)$ and $\phi_{N}(x, u, v)$ are functions holomorphic and bounded in $(x, u, v)$ for

$$
|x|<a_{N}^{\prime \prime}, \quad|u|<b_{N}^{\prime \prime}, \quad|v|<b_{N}^{\prime \prime}, \quad u^{m} v^{n} \in \mathfrak{D}\left(c_{N}^{\prime \prime}\right)
$$

and satisfying there the inequalities

$$
\left\{\begin{array}{l}
\left|\phi_{N}(x, u, v)\right| \leqq K_{N}\left(|x|^{N}+|x||v|^{N-1}+|u||v|^{N-1}\right) \\
\left|\phi_{N}(x, u, v)\right| \leqq K_{N}\left(|x|^{N}+|x||v|^{N-1}+|v|^{N}\right)
\end{array}\right.
$$

The proof of this auxiliary theorem can be carried out in quite a similar way as in 31-35. From this we can obtain the conclusion of Theorem 5.2.2 for the case when $n>0$.

39. Remark on the case when $n=0$.

In this case, the conclusion of the auxiliary theorem is still valid if we replace the domain (29.1) ${ }_{N}$ by a domain of the form $(29.2)_{N}$

$$
|x|<a_{N}^{\prime \prime}, \quad|v|<b_{N}^{\prime \prime}, \quad u^{m} \in \mathfrak{D}\left(c_{N}^{\prime \prime}\right) .
$$

Therefore, we see the existence of a solution $(Y(x, U, V)$, $Z(x, U, V))$, where

$$
\left\{\begin{array}{l}
Y(x, U, V)=P_{N}(x, U, V)+\phi_{N}(x, U, V), \\
Z(x, U, V)=Q_{N}(x, U, V)+\psi_{N}(x, U, V)
\end{array}\right.
$$

are asymptotically represented by the formal power series solution (II. 2) in 14 for $(x, U, V)$ in the domain (29.2) with $a_{0}^{\prime \prime}=\sup a_{N}^{\prime \prime}, b_{0}^{\prime \prime}=\sup b_{N}^{\prime \prime}, c_{0}^{\prime \prime}=\sup c_{N}^{\prime \prime}$. But, in the present case, these asymptotic expansions are uniformly convergent for (29.2). 
Indeed, the point $x=0, v=0$ is an inner point of the domain (29. 2) and, consequently, the functions $Y(x, u, v)$ and $Z(x, u, v)$ are holomorphic at $x=v=0$ with respect to two variables $x$ and $v$.

Section IV. Proof of Theorem 5.3.1.

40. Outline of the proof.

We make a transformation of the form

$$
y=\eta+P_{N}(x, U, V), \quad z=\zeta+Q_{N}(x, U, V),
$$

where

$$
\left\{\begin{array}{l}
P_{N}(x, u, v)=u\left(1+P_{0}^{(1)}(u, v)\right)+\sum_{j=1}^{N-1} P_{j}^{(1)}(u, v) x^{j} \\
Q_{N}(x, u, v)=v\left(1+Q_{0}^{(1)}(u, v)\right)+\sum_{j=1}^{N-1} Q_{j}^{(1)}(u, v) x^{j}
\end{array}\right.
$$

Then, the transformed system can be written as

$$
x \eta^{\prime}=F_{N}(x, U, V, \eta, \zeta), \quad x \zeta^{\prime}=G_{N}(x, U, V, \eta, \zeta)
$$

and admits a formal solution of the form

$$
\eta \sim \sum_{j=N}^{\infty} P_{j}^{(1)}(U, V) x^{j}, \quad \zeta \sim \sum_{j=N}^{\infty} Q_{j}^{(1)}(U, V) x^{j} .
$$

Also, it is found that

1) $F_{N}(x, u, v, \eta, \zeta)$ and $G_{N}(x, u, v, \eta, \zeta)$ are functions holomorphic and bounded in $(x, u, v, \eta, \zeta)$ for

(40.5) $|x|<a_{0},|u|<b_{0},|v|<b_{0}, u^{m} v^{n} \in \mathfrak{D}\left(c_{0}\right),|\eta|<b_{1},|\zeta|<b_{1}$;

2) $F_{N}(x, u, v, \eta, \zeta)$ and $G_{N}(x, u, v, \eta, \zeta)$ satisfy there the inequalities

$$
\max \left(\left|F_{N}\right|,\left|G_{N}\right|\right) \leqq A(|\eta|+|\zeta|)+B_{N}|x|^{N}
$$

and

$$
\begin{aligned}
\mid F_{N}\left(x, u, v, \eta_{1}, \zeta_{1}\right) & -F_{N}\left(x, u, v, \eta_{2}, \zeta_{2}\right) \mid \\
& \leqq A\left|\eta_{1}-\eta_{2}\right|+A\left|\zeta_{1}-\zeta_{2}\right|
\end{aligned}
$$


$\left(40.7^{\prime}\right)$

$$
\begin{aligned}
\mid G_{N}\left(x, u, v, \eta_{1}, \zeta_{1}\right)- & G_{N}\left(x, u, v, \eta_{2}, \zeta_{2}\right) \mid \\
& \leqq A\left|\eta_{1}-\eta_{2}\right|+A\left|\zeta_{1}-\zeta_{2}\right|
\end{aligned}
$$

In the present case, the inequalities (40.6), (40.7) and $\left(40.7^{\prime}\right)$ are satisfied without any conditions such as (38.8).

Under these conditions we can prove, by the same reasoning as in 31-35, the following auxiliary theorem:

Auxiliary Theorem. The system (40.3) possesses one and only one solution $\left\{\phi_{N}(x, U, V), \phi_{N}(x, U, V)\right\}$ such that $\phi_{N}(x, u, v)$ and $\phi_{N}(x, u, v)$ are functions holomorphic and bounded in $(x, u, v)$ for

$$
|x|<a_{N}^{\prime \prime}, \quad|u|<b_{N}^{\prime}, \quad|v|<b_{N}^{\prime \prime}, \quad u^{m} v^{n} \in \mathfrak{D}\left(c_{N}^{\prime \prime}\right)
$$

and, moreover, satisfying there the inequality

$$
\begin{gathered}
\max \left\{\left|\phi_{N}(x, u, v)\right|, \psi_{N}(x, u, v) \mid\right\} \leqq K_{N}|x|^{N}, \\
a_{N}^{\prime N} K_{N}<b_{1} .
\end{gathered}
$$

By virtue of this theorem, we can assert that the functions $Y_{N}(x, u, v)$ and $Z_{N}(x, u, v)$, where

$$
\left\{\begin{array}{l}
Y_{N}(x, u, v)=P_{N}(x, u, v)+\phi_{N}(x, u, v), \\
Z_{N}(x, u, v)=Q_{N}(x, u, v)+\phi_{N}(x, u, v),
\end{array}\right.
$$

are independent of $N$ for any positive integer $N$ and defined in the domain (29.1) with $a_{0}^{\prime \prime}=\sup a_{N}^{\prime \prime}, b_{0}^{\prime \prime}=\sup b_{N}^{\prime \prime}, c_{0}^{\prime \prime}=\sup c_{N}^{\prime \prime} . \quad$ Since $x=0$ is an inner point of the domain (29.1), these functions are developable there in the uniformly convergent power series (III. $\left.1^{\prime}\right)$ in 29. Clearly, $\left(Y_{N}(x, U, V), Z_{N}(x, U, V)\right)$ represents a solution of the system (1.1). This proves Theorem 5.3.1.

Section V. Proof of Theorems 5.3.2 and 5.3.3.

\section{Outline of the proof.}

We discuss the proof of Theorem 5.3.2 only, because Theorem 5.3.3 can be proved in a similar way. Set 


$$
\left\{\begin{array}{l}
P_{N}(x, u, v)=\sum_{l=0}^{N-1} P_{l}^{(2)}(x, u) v^{l}, \\
Q_{N}(x, u, v)=\sum_{l=0}^{N-1} Q_{l}^{(2)}(x, u) v^{l} .
\end{array}\right.
$$

Let us make a transformation of the form

$$
y=\eta+P_{N}(x, U, V), \quad z=\zeta+Q_{N}(x, U, V) .
$$

We write the transformed system as

$$
x \eta^{\prime}=F_{N}(x, U, V, \eta, \zeta), \quad x \zeta^{\prime}=G_{N}(x, U, V, \eta, \zeta) .
$$

Then we see immediately that this system admits a formal solution of the form

$$
\eta \sim \sum_{l=N}^{\infty} P_{l}^{(2)}(x, U) V^{\imath}, \quad \zeta \sim \sum_{l=\Lambda N}^{\infty} Q_{l}^{(2)}(x, U) V^{l}
$$

Furthermore, we can easily verify that, if $n>0$,

1) $F_{N}(x, u, v, \eta, \zeta)$ and $G_{N}(x, u, v, \eta, \zeta)$ are functions holomorphic and bounded in $(x, u, v, \eta, \zeta)$ for

$$
|x|<a_{0}, \quad|u|<b_{0}, \quad|v|<b_{0}, \quad|\eta|<b_{1}, \quad|\zeta|<b_{1} ;
$$

2) they satisfy the inequalities

$$
\begin{aligned}
\left|F_{N}(x, u, v, \eta, \zeta)\right| \leqq & A\left(|x|+|u|^{m}|v|^{n}\right)|\eta| \\
& +A\left(|x|+|u|^{m+1}|v|^{n-}\right)^{1}|\zeta| \\
& +B_{N}\left(|x| \cdot|v|^{N}+|u|^{m}|v|^{n} \cdot|u||v|^{N-1}\right),
\end{aligned}
$$

$$
\begin{aligned}
\left|G_{N}(x, u, v, \eta, \zeta)\right| \leqq & A\left(|x|+|u|^{m-1}|v|^{n+1}\right)|\eta| \\
& +A\left(|x|+|u|^{m}|v|^{n}\right)|\zeta| \\
& +B_{N}\left(|x| \cdot|v|^{N}+|u|^{m}|v|^{n} \cdot|v|^{N}\right)
\end{aligned}
$$

and, moreover, we have the inequalities (30.10) and (30.10') provided that the conditions

$$
|\eta| \leqq C(|x|+|u|), \quad|\zeta| \leqq C(|x|+|v|)
$$

are satisfied, where $C$ is a positive constant.

By the same reasoning as in 31-35, we can establish the following Auxiliary Theorem:

Auxiliary Theorem. There exists one and only one solution 
$(\eta, \zeta)=\left(\phi_{N}(x, U, V), \dot{\varphi}_{N}(x, U, V)\right)$ such that $\phi_{N}(x, u, v)$ and $\phi_{N}(x, u, v)$ are functions holomorphic and bounded in $(x, u, v)$ for $(29.1)_{N} \quad|x|<a_{N}^{\prime \prime}, \quad|u|<b_{N}^{\prime \prime}, \quad|v|<b_{N}^{\prime \prime}, \quad u^{m} v^{n} \in \mathfrak{D}\left(c_{N}^{\prime \prime}\right)$

and we have

$$
\begin{aligned}
& \left|\phi_{N}(x, u, v)\right| \leqq K_{N}\left(|x||v|^{N-1}+|u||v|^{N-1}\right) \\
& \left|\phi_{N}(x, u, v)\right| \leqq K_{N}\left(|x||v|^{N-1}+|v|^{N}\right)
\end{aligned}
$$

with

$$
\left(a_{N}^{\prime \prime} b_{N}^{\prime N-1}+b_{N}^{\prime N}\right) K_{N}<b_{1} .
$$

From this we see the existence of a solution $\{\Phi(x, U, V)$, $\Psi(x, U, V)\}$ of the system (1.1), where the functions $\Phi(x, u, v)$ and $\Psi(x, u, v)$ are asymptotically represented by the formal series (III. $2^{\prime}$ ) in 29 for the domain (29.1) with $a_{0}^{\prime \prime}=\sup a_{N}^{\prime \prime}, b_{0}^{\prime \prime}=\sup b_{N}^{\prime \prime}$, $c_{0}^{\prime \prime}=\sup c_{N}^{\prime \prime}$. Especially, in the case when $n=0$, these asymptotic expansions are uniformly convergent for

$$
|x|<a_{0}^{\prime \prime}, \quad|v|<b_{0}^{\prime \prime}, \quad u^{m} \in \mathfrak{D}\left(c_{0}^{\prime}\right) .
$$

Thus Theorem 5.3.2 has been proved.

\section{Return to Theorem 5.2.2 for the case $n=0$.}

We can obtain from the conclusion of Theorem 5.3.2 for the case $n=0$ the conclusion of Theorem 5.2.2 for the case $n=0$.

Indeed, since the point $x=0, v=0$ is an inner point of the domain (29.2), the functions $\Phi(x, u, v)$ and $\Psi(x, u, v)$ are holomorphic at $(0,0)$ with respect to two variables $x$ and $v$. Consequently, they are expanded in uniformly convergent power series of $x$ and $v$. On the other hand, as can be easily seen from the determination of the coefficients of the formal solution (III. 2) ((III. $\left.2^{\prime}\right)$ ) (which, in the present case, is uniformly convergent), such doulbe power series of $x$ and $V$ coincide formally with the formal solution (II. 2) ((II. $\left.2^{\prime}\right)$ ). It follows then that the formal solution (II. 2) is uniformly convergent in the domain 
(29.2) (where $u$ and $v$ must be replaced by $U$ and $V$ respectively).

This proves Theorem 5.2.2 for the case $n=0$.

Remark. Similarly, we can obtain from the conclusion of Theorem 5.3.3 for the case $m=0$ the conclusion of Theorem 5.2.3 for the case $m=0$.

\section{REFERENCES}

M. Hukuhara : [1] Sur les points singuliers d'une équation différentielle ordinaire du premier ordre, I. Mem. Fac. Eng. Kyushu Imp. Univ. 8 (1937), 203-247.

: [2] Sur les points singuliers d'une équation différentielle ordinaire $d u$ premier ordre, II. Proc. Phys. Math. Soc. Japan 20 (1938), 157-189.

: [3] Sur les points singuliers d'une équation différentielle ordinaire du premier ordre, III. Ibid. 20 (1938), 409-441.

_- [4] Sur les points singuliers d'une équation différentielle ordinaire du premier ordre, IV. Ibid. 20 (1938), 865-907.

: [5] Sur les points singuliers d'une équation différentielle ordinaire du premier ordre, V. Mem. Fac. Sci. Kyushu Imp. Univ. 3 (1944), 67-73.

: [6] Pri singula punkto de la ordinara diferentiala ekvacio de una ordo. Ibid. 3 (1949), 9-21.

: [7] Pri la elvolvado de solvo de diferentialaj ekvacioj en la chirkauajho de ilia singula punkto. Ibid. 4 (1949), 1-7.

: [8] Êquations différentielles ordinaires du premier ordre dans le champ complex (with T. Kimura and Mrs. T. Matuda). Publ. Math. Soc. Japan 7, Tokyo, Math. Soc. Japan, 1961. Theory of the ordinary differential equations (in Japanese), Iwanami Zensyo, Tokyo, 1952.

: [9] Sur les points singuliers des ćquations différentielles linéaires, III. Mem. Fac. Sci. Kyushu Imp. Univ. 2 1942, 125-137.

: [10] Renzokuna Kansû no Zoku to Syazô. Mem. Fac. Sci. Kyushu Univ. Ser. A, 5 (1950), 61-63.

M. Iwano : [1,2] Intégration analytique d'un système d'ćquations diffćrentielles non linéaires dans le voisinage d'un point singulier, I, II. Annali Mat. Pura Appl. Serie IV, 44 (1957), 261-292, 47 (1959), 91-150.

: [3] On a singular point of Briot-Bouquet type of a system of ordinary nonlinear differential equations. Comment. Math. Univ. Sancti Pauli, 11 (1963), 37-78.

T. Kimura : [1] Sur une généralisation d'un théorème de Malmquist, I, II, III. Comment. Math. Sancti Pauli, 2 (1953), 23-28, 3 (1955), 97-107, 4 (1955), 25-41. 
J. Malmquist : [1] Sur les fonctions d'un nombre fini de branches définies par les équations différentielles du premier ordre. Acta Math. 36 (1913), 297-343.

\author{
Mathematics Research Center \\ United States Army, University \\ of Wisconsin \\ and \\ Department of Mathematics \\ Tokyo Metropolitan University
}

April 5, 1966 Madison 
